Microglial LRP1 modulates neuroinflammation via control of the NF-kB pathway in autoimmunity

\author{
Tzu-Ying Chuang \\ Taipei, Taiwan
}

B.A. cum laude, Chemical and Physical Biology, Harvard College, 2010

\begin{abstract}
A Dissertation presented to the Graduate Faculty of the University of Virginia in Candidacy for the degree of

Doctor of Philosophy
\end{abstract}

Department of Molecular and Cellular Basis of Disease (Pathology)

University of Virginia

December 2016 


\begin{abstract}
Multiple sclerosis (MS) is the most common demyelinating disease of the central nervous system (CNS), characterized by immune cell infiltration and inflammation. While $\mathrm{T}$ cells are known contributors to MS pathology, the precise function of CNS resident and peripheral infiltrating myeloid cells is more controversial. In this work I delineate the myeloid cell function of LRP1, a scavenger receptor crucial for myelin clearance and the inflammatory response, in the context of MS. I find that LRP1 expression is increased in the MS lesion in comparison to the surrounding healthy tissue. Using two genetic mouse models, I show that deletion of LRP1 in microglia, but not in peripheral macrophages, negatively impacts the progression of experimental autoimmune encephalomyelitis (EAE), an animal model of MS. I further demonstrate that this increase in EAE disease severity is not due to haplodeficiency of the $C x 3 c r 1$ locus. Furthermore, microglia lacking LRP1 adopt a pro-inflammatory phenotype characterized by amoeboid morphology and increased production of the inflammatory mediator TNF $\alpha$. I also show that LRP1 inhibits NF- $\kappa$ B activation in myeloid cells via a MyD88 dependent mechanism. Together, my data suggests that LRP1 functions in microglia to keep these cells in an anti-inflammatory and neuroprotective status during inflammatory insult, including experimental autoimmune encephalomyelitis and potentially in MS. I also initiated studies exploring the role of LRP1 as a phagocytic receptor of myelin during MS using a combination of in vitro and in vivo models. This work will provide a foundation for future studies regarding the role of LRP1 in mediating the crosstalk between phagocytosis and inflammation.
\end{abstract}




\section{Table of Contents}

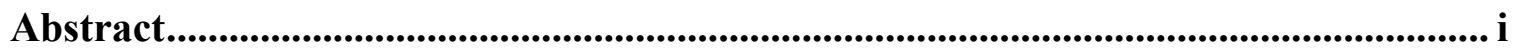

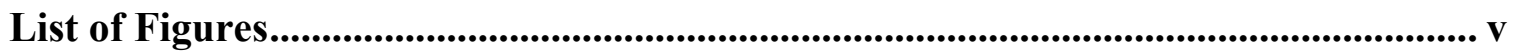

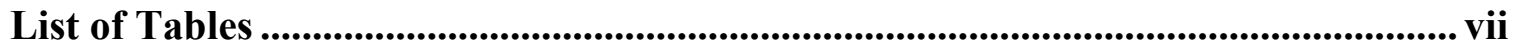

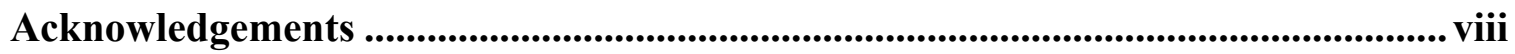

Chapter 1: INTRODUCTION

Multiple Sclerosis................................................................................................................................. 1

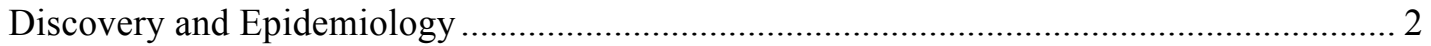

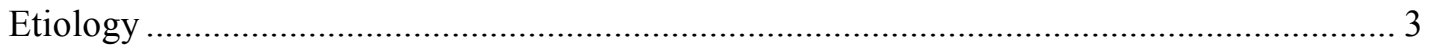

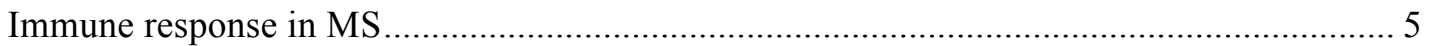

Animal Models of MS: Successes and Limitations ..................................................................... 6

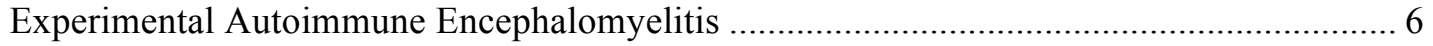

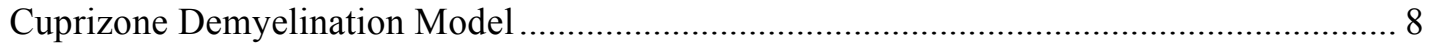

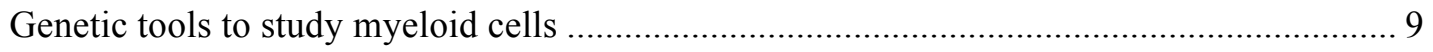

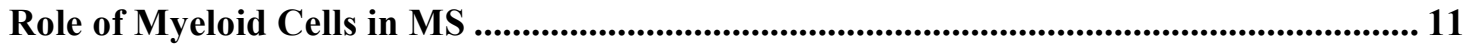

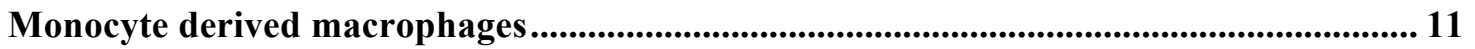

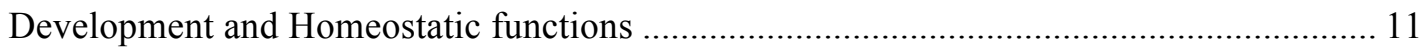

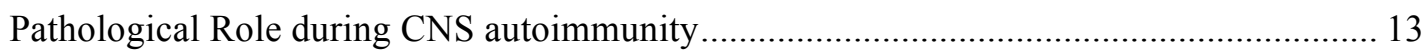

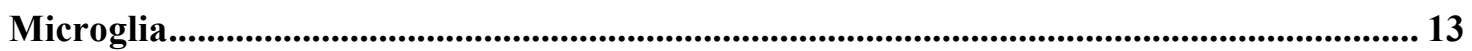

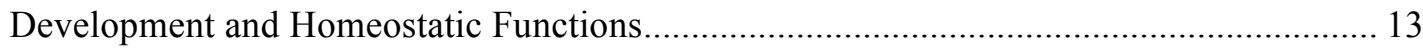

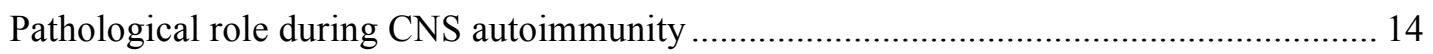

Molecular mediators of myeloid inflammation during MS: NF-кB .............................. 16

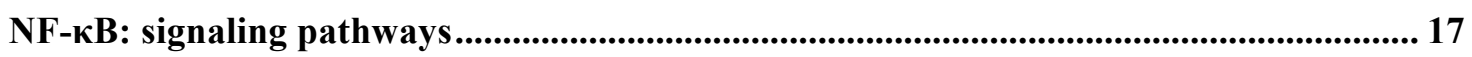

The crosstalk between phagocytosis and inflammation ...................................................... 19

Phagocytosis in MS: a restorative, anti-inflammatory mechanism .................................... 21

Toll like receptors in phagocytosis ............................................................................................. 22

Scavenger receptors in phagocytosis: TREM2 ..................................................................... 23

LRP1: a key regulator of inflammation and phagocytosis ................................................. 24

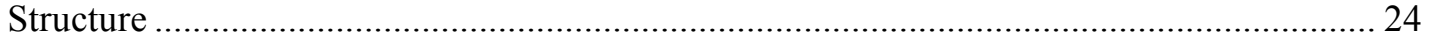

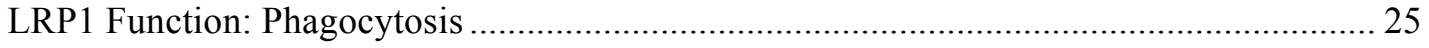

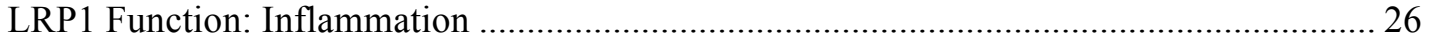




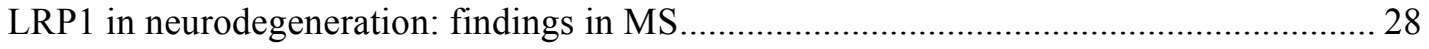

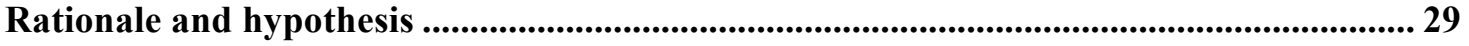

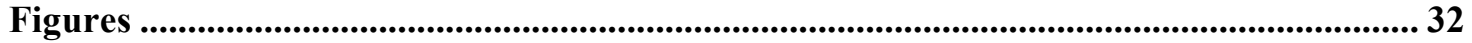

Chapter 2: MATERIALS AND METHODS............................................................... 38

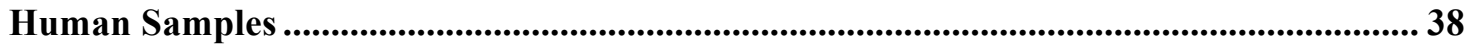

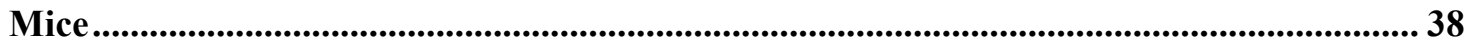

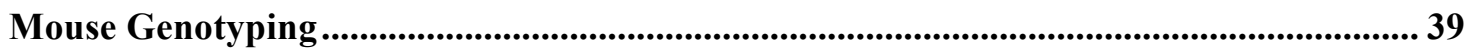

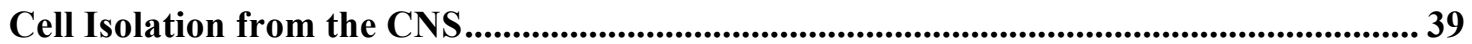

Experimental Autoimmune Encephalomyelitis (EAE) ...................................................39

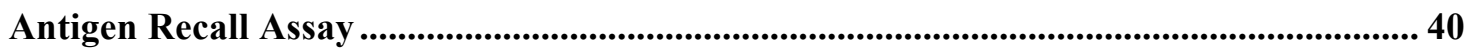

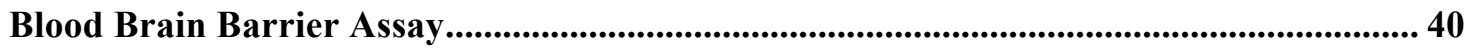

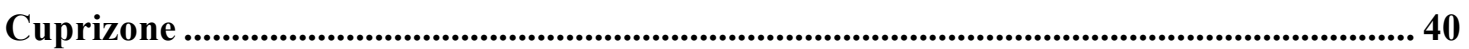

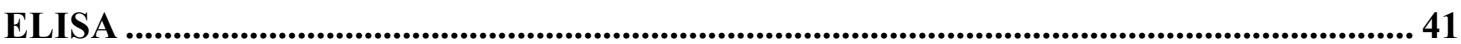

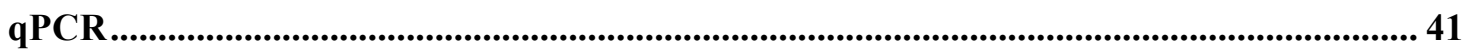

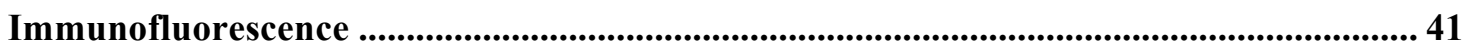

Immunohistochemistry ...................................................................................................................... 42

Preparation of Bone Marrow Derived Macrophages................................................................. 42

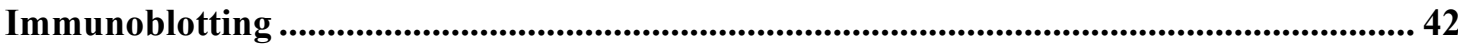

Flow Cytometry/Fluorescence Activated Cell Sorting ................................................................. 43

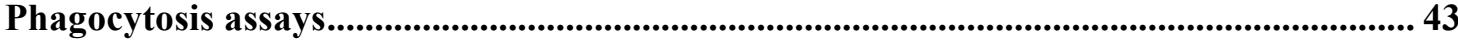

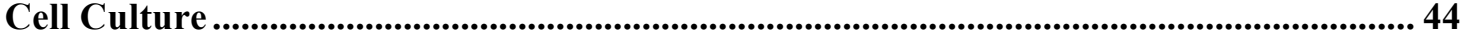

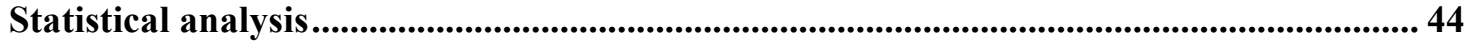

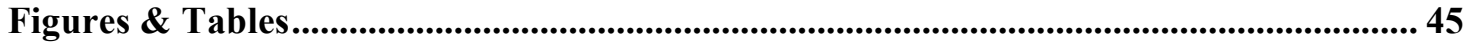

Chapter 3: LRP1 EXPRESSION IN MICROGLIA IS PROTECTIVE DURING

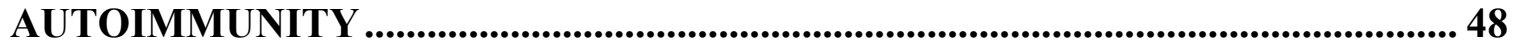

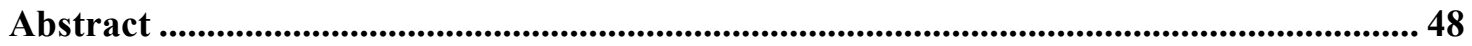

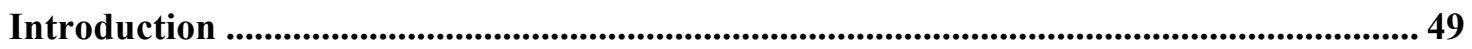

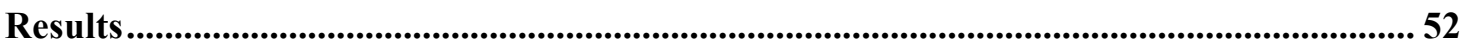

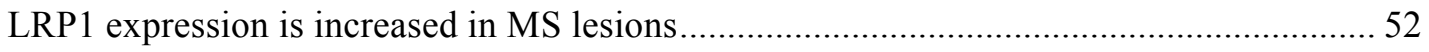

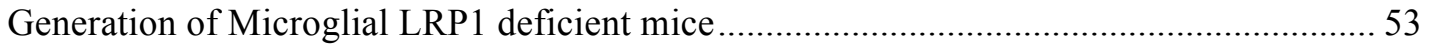

Microglial, but not peripheral macrophage LRP1, is protective during experimental

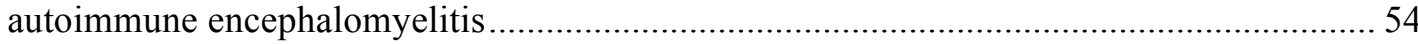

Demyelination and peripheral immune cell recruitment are elevated in mice lacking LRP156 
Lymphocyte activation is not affected in the absence of LRP1.....

Function of the blood brain barrier is intact in mice lacking microglial LRP1 .................... 57

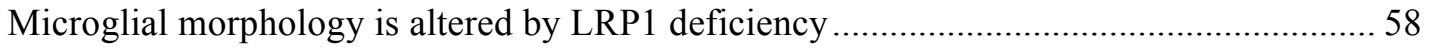

Microglia lacking LRP1 have a proinflammatory signature …........................................... 59

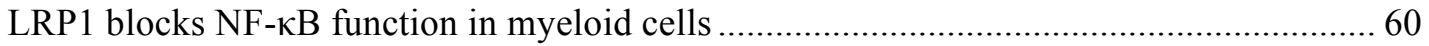

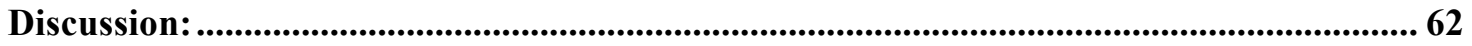

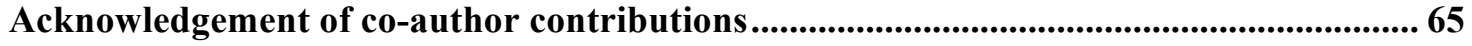

Chapter 4: LRP1 IS ESSENTIAL FOR THE PHAGOCYTOSIS OF MYELIN AND

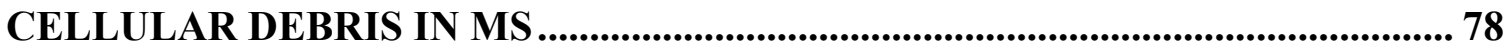

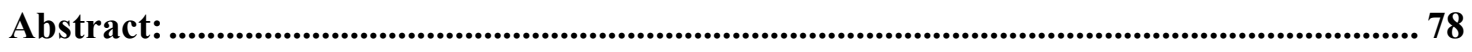

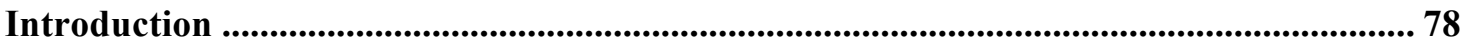

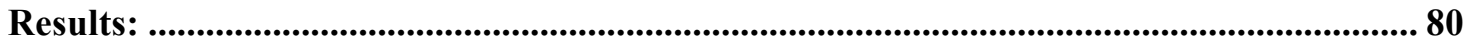

LRP1 deficient myeloid cells have impaired myelin phagocytosis, but not of foreign

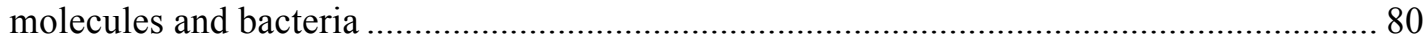

During EAE, LRP1 deficient microglia may have lysosomal deficits related to phagocytosis

$\mathrm{Cx} 3 \mathrm{cr} 1^{\mathrm{cre}} \mathrm{LRP} 1^{\mathrm{fl} / \mathrm{fl}}$ mice have deficits in oligodendrocyte proliferation in a cuprizone model

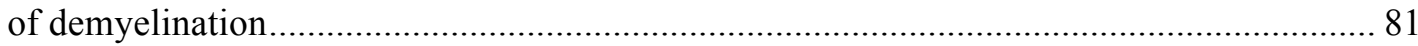

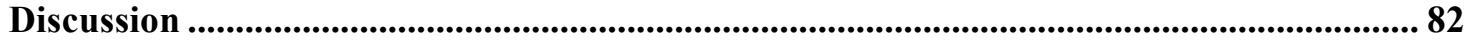

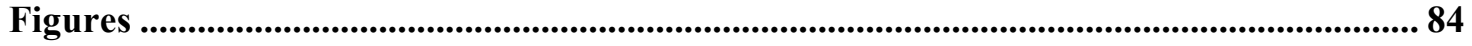

Chapter 5: SUMMARY ............................................................................................. 87

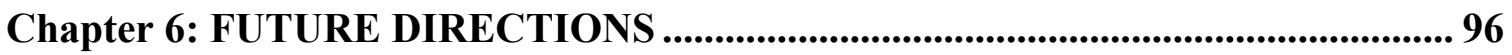

How does LRP1 inhibit NF-kB? ........................................................................................... 96

Is the inflammatory phenotype of LRP1 deficient microglia exacerbated by a phagocytic

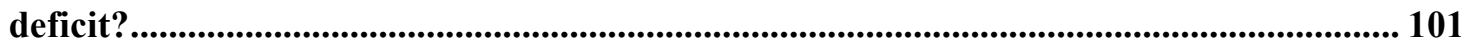

Are there lysosomal changes related to phagocytic defects in LRP1 deficient microglia? 105

How does microglial LRP1 deficiency affect remyelination? .............................................. 108

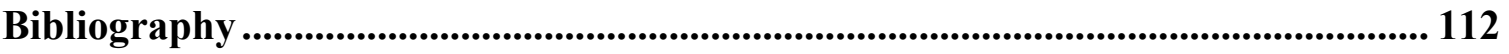




\section{List of Figures}

Figure 1: History of MS and Current Therapies 32

Figure 2: Genetic tools for interrogating LRP1 function on myeloid cells of different origin during EAE 33

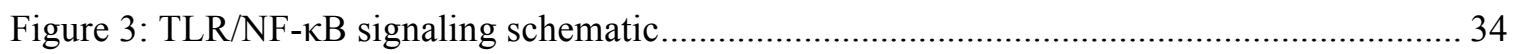

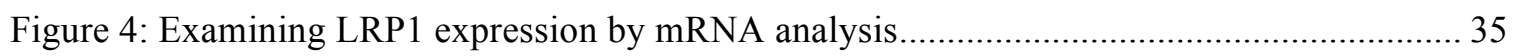

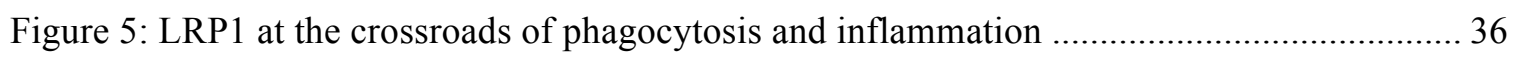

Figure 6: Experimental protocol for experimental autoimmune encephalomyelitis (EAE) ......... 45

Figure 7: Flow cytometry gating strategy to identify macrophages, microglia and lymphocytes. 46

Figure 8: LRP1 expression is increased in MS lesions

Figure 9: LRP1 is deleted from microglia of the novel mouse strain Cx3 $3 \mathrm{cr}^{\mathrm{crER}}-\mathrm{LRP} 1^{\mathrm{fl} / \mathrm{fl}}$, while in $\mathrm{Cx} 3 \mathrm{cr} 1^{\mathrm{cre}}$-LRP $1^{\mathrm{fl} / \mathrm{fl}}$ mice, LRP1 is deleted from both the microglia and the bone marrow-derived macrophages.

Figure 10: Deletion of LRP1 from microglia, but not from peripheral myeloid cells, exacerbates EAE progression.

Figure 11: Higher mycobacterium stimulus in $\mathrm{LysM}^{\mathrm{cre}}$-Lrp $1^{\mathrm{fl} / \mathrm{fl}}$ mice and CX3CR1 haplodeficiency do not affect EAE progression.

Figure 12: Microglial LRP1 deficient mice have increased numbers of immune cells in the CNS during EAE.

Figure 13: Immune response and blood-brain barrier permeability are not altered in Microglial LRP1 deficient mice.

Figure 14: LRP1 deficient microglia have an amoeboid morphology....................................... 73

Figure 15: LRP1 deficiency in microglia does not alter weight loss after LPS treatment............. 74

Figure 16: LRP1 deficient myeloid cells have a pro-inflammatory signature ............................. 75

Figure 17: LRP1 deficient microglia have an increased pro-inflammatory signature in EAE...... 76

Figure 18: LRP1 is an inhibitor of NF- $\mathrm{BB}$ through the MyD88 pathway..................................... 77 
Figure 19: LRP1 deficient myeloid cells are deficient in phagocytosis of myelin, but not of TLR ligands 84

Figure 20: LRP1 deficient microglia have altered lysosomal gene regulation 85

Figure 21: Mice with LRP1 deficient microglia have less oligodendrocyte progenitor cells during

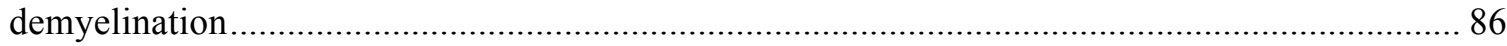

Figure 22. Schematic of the proposed interaction between LRP1 and TRAF6/A20 ................. 111 


\section{List of Tables}

Table 1: LRP1 functions as a scavenger receptor for multiple ligands ......................................... 34

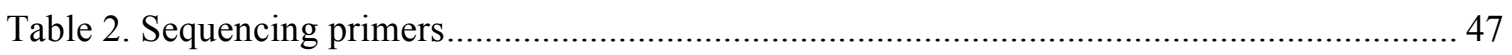

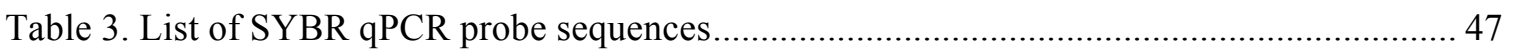

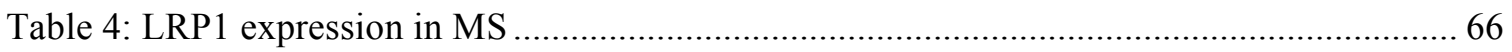




\section{Acknowledgements}

"It takes a village to raise a child." Although it would seem cultivating a $\mathrm{PhD}$ candidate would be easier, the development from a scientific tadpole to a grown frog required the care, time and attention of many individuals to whom I am eternally grateful.

First and foremost, the most important driver of my scientific work is without a doubt my scientific mentor and life coach, Alban Gaultier. I honestly still cannot believe how lucky I am to have joined his lab. He challenges and inspires his students in asking the rigorous scientific questions while being a truly compassionate human being. I look up to him and respect him as a researcher, philosopher, and mentor for life.

My fellow graduate students, Scott Seki, the letter A Rosen, Anthony FernándezCastañeda, and Ioana Marin, have been instrumental to my success. I have learned so much from each of them, ranging from baby steps of flow cytometry to the fine art of writing. Thank you for the experimental assistance, invigorating conversations, constant camaraderie and friendship that has kept me sane and helped me grow throughout my time in graduate school. Jennifer Goertz, who provided excellent editorial assistance, made the writing process infinitely easier.

The acknowledgements would be incomplete without mentioning my committee:

Drs. James Mandell, Janet Cross, and Tajie Harris. I appreciate the intellectual rigor and thoughtful input they invested in my development throughout my $\mathrm{PhD}$ work, helping me to become a thorough and incisive scientist. I would also like to thank the Department of Neuroscience, the MCBD, and the MSTP program, for giving me this opportunity to pursue a degree in biomedical science in conjunction with my medical studies. Becoming 
a physician researcher is truly an honor that I hope to embody by always asking the question of "Why?" in my heart while considering potential therapeutic options.

To the supportive friends I have made along the way in graduate school, for the trips, board games, music, dessert nights, hikes and love which have restored my sanity and rejuvenated my soul: Allissia Gilmartin, Lucia Leitner, Nenja Kruger, as well as the members of the Taiwanese Graduate Student Association and the First Harmonics. My parents, Heng-Sheng Chuang (莊恆盛) and Chin-Yu Hsu (許錦玉), who have been pillars of support my whole life and constantly encouraged me, instilling in me the value of hard work, dedication, and a love of learning. I saw this in their own life stories, how they went from childhoods as cow herders and farmers in the bucolic yet resourcescarce countryside to becoming warm, enthusiastic ambassadors of Taiwan to the world. Also to my grandparents who helped raise me as a child, who whispered fiercely to me that money is made and lost, but education is something no one can take away from you. I have learned that education gives me choices, and it is a precious opportunity that not everyone is afforded. For that, I am extremely grateful. I would also like to thank my younger brother, Allan (莊子賢), who has been extremely understanding of my sometimes dubiously organized experimental schedule, indulged my mercurial moods of graduate school, as well as being an excellent travel companion and chef. He has seen and heard first hand all about how a high success rate of scientific discovery is $20 \%$.

Finally, thank you to the many mice that contributed to my otherwise unrecognized degree: mouse doctorate, M.D.

August 5, 2016

Tzu-Ying Chuang 莊子瑩 


\section{Chapter 1: INTRODUCTION}

\section{Multiple Sclerosis}

The central nervous system (CNS) is instrumental to functions ranging from basic movement to complex cognition. Thus, diseases that affect the CNS have social and economic implications beyond that which can be illustrated by epidemiological data such as prevalence and incidence. Neurological disorders account for $7.1 \%$ for the global burden of disease in terms of prevalence and incidence, and Multiple Sclerosis (MS) accounts for just $0.6 \%$ of this disease burden ${ }^{1}$, as measured in disability associated life years (DALY). Globally the prevalence of MS is $~ 30$ people per 100,000. However, these measurements do not account for the extremely high economic, psychological, and social costs of MS for individuals, their caretakers, and their families. On average, MS ranks second in highest direct all-cause medical costs for chronic conditions, behind congestive heart failure ${ }^{2}$. It is the most common CNS demyelinating disorder, and since disease onset typically occurs during the second or third decades of a patient's life, MS causes lifelong burdens for young adults just beginning their careers and families. Within 10-15 years of diagnosis, over $80 \%$ of patients will experience disability ${ }^{3}$.

Furthermore, the only available therapies for MS are disease-modifying agents, which only serve to slow the progression of disease. There is still no identified etiology for the initiation of pathophysiology in MS, and so it is equally hard to develop curative treatments. Therefore, future research into the pathogenesis of MS juxtaposes the need to understand biological processes as well as the need to find pharmacologically viable targets for therapeutic development. 


\section{Discovery and Epidemiology}

Multiple Sclerosis is characterized by episodic orchestrated immune attacks on the myelin sheaths of axons in the CNS. Early stages of disease are distinguished by a multifocal loss of myelin with relative preservation of axons. In historical medical records, reports of patients with MS-like symptoms date back to the $14^{\text {th }}$ century. However, it was only in 1868 that Dr. Jean Marie Charcot first correlated the clinical symptoms of his patients with lesions in the post-mortem CNS. He observed "la sclérose en plaques" in autopsy samples from his patients who had been suffering from vision and movement problems ${ }^{4}$ (Fig. 1) and is often credited with the first histopathological characterization of MS.

The patient population is predominately female with a 3:1 ratio, and global incidence of disease is over two million ${ }^{5}$. There is a higher likelihood of becoming diagnosed with MS in Caucasian populations of Northern European descent, but patients with Asians and African descent can also present with MS. There are clear genetic associations with MS, as concordance rate of MS in monozygotic twins is $30 \%$ but decreases to $5 \%$ in dizygotic twins. However, there are still no clear genetic predictors or biomarkers for confirming diagnosis or assessing prognosis in MS. Some immune related genes have been associated with disease initiation, but none are necessary nor sufficient for either diagnosis or prognosis ${ }^{6}$.

MS presents as several different clinical forms of disease. The most common form, Relapsing-Remitting MS (RRMS), affects around 85\% of all MS patients. Patients experience an acute onset of symptoms, termed an MS attack, which can present as visual, motor, sensory, cognitive, and/or autonomic dysfunction. Most individuals with 
RRMS will eventually develop secondary progressive MS (SPMS), characterized by absence of further remissions and relentless worsening of disease. Although the transition between these stages of disease is still poorly understood, it is thought that the axonal injury caused by long-term myelin loss and absence of complete repair contributes to the progression towards SPMS. Still other patients develop different forms of MS called primary progressive MS (PPMS) and progressive relapsing MS (PRMS) ${ }^{7}$. The distinct types of MS indicate that many questions regarding the disease remain, ranging from genetic predisposition and pathophysiological diversity between the different types of disease as well as how, if any, transitions between different types of disease occur.

\section{Etiology}

Although the true etiology of MS is still unknown, there are many hypotheses concerning the cause of disease. The incidence of disease is affected by geographic location and age, generally increasing with distance from the equator. Studies based in several different countries, including the US, Australia, Israel, and the West Indies have illustrated that childhood location is highly correlated to risk of disease. Migration from a high-risk to low-risk location before the age of 15 is correlated with a decreased risk of developing MS, and the opposite, moving from a low-risk to high risk location, correlates with an increased risk of developing $\mathrm{MS}^{6,8}$. Epstein-Barr virus infection at an early age and genetics are also linked to disease onset. MS has a recurrence rate of $20 \%$ for monozygotic twins, but the rate decreases to $3-5 \%$ in dizygotic twins, which suggests a polygenic disease inheritance pattern. Analysis on multiple MS patient GWAS studies from international research consortiums shows that most of the genes associated with patients are related to immune regulation, such as $H L A-D R B 1, I L 12, I L 2 R$, and $C D 86^{9}$. 
The link between immune dysfunction and MS is further underscored by the fact that all current treatments target different mechanisms of immune involvement in MS, possibly mitigating immune activity in the CNS and controlling damage caused by inflammation.

Since the etiology of MS remains unidentified, there are various theories concerning the origin of disease. Broadly speaking these can be termed the "outside-in" and the "inside-out" model. The more traditional "outside-in" model suggests that immune dysregulation is the inciting event in disease initiation. An autoimmune reaction is initiated, perhaps by a still unidentified viral infection activating peripheral selfreactive $\mathrm{T}$ cells through molecular mimicry. The $\mathrm{T}$ cells become auto-reactive and cross into the CNS during blood brain barrier dysfunction, causing damage through inflammatory activity, secretion of cytokines and recruitment of other immune cells. This inflammatory reaction then results in further demyelination and tissue injury ${ }^{10}$.

The "inside-out" model argues instead that there is a primary failure of the oligodendrocytes in the CNS, which are the cells responsible for the production of myelin $^{11}$. Traka et al. developed a mouse where oligodendrocytes are killed via tamoxifen inducible PLP1 promoter specific activation of diphtheria toxin (DTA), leading to extensive myelin and axonal loss with extensive $\mathrm{T}$ cell infiltration into the CNS. This suggests that primary oligodendrocyte death can trigger an adaptive autoimmune response against myelin ${ }^{12}$. Biopsies and autopsies from MS patients have also revealed the presence of myelin damage or extensive demyelination with little inflammatory infiltration ${ }^{13}$. 
Immune response in $M S$

MS attacks may be precipitated by upper respiratory infections, urinary tract infections, and stress - all instances when the immune system is primed for activation ${ }^{14}$. Close histological examination of MS lesions reveals massive infiltration of immune cells into the brain and spinal cord parenchyma. The degree of clinical disease severity is correlated with the number of immune cells present, and it is thought that the proinflammatory activity of these immune cells, especially $\mathrm{T}$ and myeloid cells, is ultimately responsible for the resulting demyelination found in MS lesions ${ }^{15}$. In a systematic review of patient lesions, Lucchinetti et al. defined four categories of lesions based on cell composition, complement activation, oligodendrocyte destruction, and myelin protein destruction ${ }^{16,17}$. The acute destruction of myelin also leads to neuronal death, as neurons lose the protective and trophic support provided by myelin ${ }^{18}$. Oligoclonal IgG is found in the cerebrospinal fluid of most MS patients, further supporting dysregulated immune activation during MS attacks.

During relapses, the immediate need is to diminish immune activity, which is currently pursued through administering steroids or plasma exchange. During the remission phase in between MS attacks, patient neurological symptoms recover to nearly baseline. The number of immune cells in the lesion decreases, perhaps due to apoptosis and drainage to local lymph nodes. Some lesions even begin remyelination, resulting in the "shadow" plaques observed on autopsy analysis. However, the long-term axon damage due to the lack of remyelination and recurrent attacks results in permanent motor and sensory disability in all MS patients ${ }^{19}$. 
Before the advent of MRI, MS lesions could only be identified post-mortem in patient brains. Modern diagnostic criteria for MS now always includes identification of at least one gadolinium enhancing, demyelinating lesion by $\mathrm{MRI}^{20}$. Within the actively demyelinating MS lesion, macrophages/microglia are the most abundant immune cell type $^{21}$. Axonal injury in MS, as measured by accumulation of amyloid precursor protein (APP), correlates with the number of macrophage-like cells within the lesion site ${ }^{22}$. Their increased abundance correlates with increased clinical severity ${ }^{23}$, highlighting the potential in modulating their inflammatory response for disease treatment.

\section{Animal Models of MS: Successes and Limitations}

In this section, I will describe the animal models and genetic tools I used during my dissertation work to explore the function of low-density lipoprotein receptor 1 (LRP1) which I introduce in more detail later - on the microglia and peripheral macrophages. First I will describe a model focused on active demyelination, the experimental autoimmune encephalomyelitis (EAE) mouse model. Second, I will describe the model focused on the remyelination process, the cuprizone mouse model. I will conclude by describing the genetic models that I have used to interrogate the function of LRP1 in myeloid cells.

\section{Experimental Autoimmune Encephalomyelitis}

Hypotheses concerning roles of specific proteins and cells involved in MS pathogenesis are often addressed with a well-established animal model of MS, Experimental Autoimmune Encephalomyelitis (EAE). The development of EAE originated in the 1920s (Fig. 1), with the observation that humans developed 
demyelinating disease from rabies virus vaccines grown on rabbit spinal cord. It was later shown that this demyelinating disease resulted from an autoimmune reaction to rabbit spinal cord contaminants in the vaccine ${ }^{24}$. This principle was applied to various animal models, including monkeys and rodents. Due to the repertoire of genes targeted by currently available rodent models and their fast propagation time, relative to primates, rodents are among the most used models of EAE today.

EAE can be induced by either "active" immunization of mice against a myelin peptide antigen, or "passive" transfer of immunogenic $\mathrm{T}$ cells that already recognize myelin peptide into a naïve animal. In this body of work I discuss the use of EAE in its active form on $\mathrm{C} 56 \mathrm{BL} / 6$ mice. Active $\mathrm{EAE}$ is induced when mice are directly subcutaneously injected with a short myelin peptide, Myelin Oligodendrocyte Glycoprotein (MOG) ${ }_{35-55}$, emulsified in Complete Freund's Adjuvant (CFA) to activate both the innate and the adaptive immune response. Mice usually begin to show signs of paralysis starting at day 9-12 and continue to show worsening disease until they reach a plateau. This model is particularly useful for studying acute initiation of inflammation and resulting demyelinating activity. As there are already a multitude of genetically modified C56BL/6 mice available, this also makes them especially useful in the context of studying roles of particular cellular compartments and/or proteins expressed on these cells.

One of the limitations of EAE in C57/BL6 is its monophasic nature. As previously mentioned, the majority of MS cases are relapsing remitting. This disease course would be more closely modeled by inducing EAE into SJL background mice. Furthermore, as the etiology of MS is still unknown, the "autoantigen" that truly initiates 
MS in humans cannot be used in these animal models. Still, work in EAE has resulted in the approval of drug therapies for MS within the last 30 years, beginning with Interferon$\beta$ in the 1980s. Current methods of delivery include injectable, oral and infused medication options for patients, including natalizumab, glatiramer acetate, and fingolimod. All current therapeutics involve diverse mechanisms of immune modulation, some of which are highlighted in Figure 1.

\section{Cuprizone Demyelination Model}

After the acute inflammatory response subsides during MS, there are varying degrees of remyelination in the MS plaque. However, the process of remyelination and its triggers remains to be elucidated. Oligodendrocyte progenitor cells (OPCs) in the healthy CNS differentiate into oligodendrocytes (OLG), which promote and produce myelin. During MS attacks there is massive oligodendrocyte death, and the differentiation of OPCs is blocked in the aftermath of the inflammatory episode ${ }^{25}$. Thus there is great interest in finding ways to promote differentiation from OPCs to OLGs in MS mouse models, in the hopes of applying this knowledge to developing therapeutics for remyelination in MS.

One of the established animal models in studying remyelination is the cuprizone mouse model, which involves feeding mice with chow containing the copper chelator cuprizone (bis-cyclohexanone oxaldihydrazone). During week 1-2 of the cuprizone diet, oligodendrocyte death and loss of myelin gene expression is observed in the corpus callosum $^{26}$. By week 4-5, massive numbers of OPCs can be detected in the largely demyelinated corpus callosum. Mice are usually analyzed at week 5 for extent of demyelination. During this processes microglia are extensively involved in phagocytosis 
and removal of damaged myelin, which is a prerequisite of successful remyelination ${ }^{26}$. There is no evidence of blood brain barrier breakdown in cuprizone treated mice, and $\mathrm{T}$ cells are not thought to be involved in disease initiation and progression ${ }^{27}$. For analysis of remyelination, cuprizone is removed from the diet at week 5 and mice examined at 2 weeks post cuprizone removal ${ }^{28}$.

How cuprizone induces demyelination is still unclear, although mechanisms such as the stress response have been suggested ${ }^{29}$. It has been shown, however, that supplementing copper into mouse chow with cuprizone does not alter the extent of demyelination $^{26}$. The accumulation of microglia/macrophages into the demyelinating lesion suggests that local microglia may be proliferating and/or that peripheral macrophages may be contributing to the population of myeloid cells in the lesion ${ }^{30}$. Indeed, peripheral GFP+ macrophages were recruited to the CNS during cuprizoneinduced demyelination despite an intact blood brain barrier ${ }^{31}$.

The corpus callosum has been extensively studied in the cuprizone model as it is a white matter region with easy accessibility and clear, consistent demyelination. However, as increasing understanding of gray matter demyelination occurs, it is expected that other regions of the brain will also be studied in more depth using this model.

Genetic tools to study myeloid cells

The current tools for studying myeloid cell specific protein function include LysM $M^{\text {cre }}$ and $C x 3 c r 1^{\text {cre }}$. The LysMcre knock-in/knockout allele has a nuclear localized Cre inserted in the first coding ATG of the lysozyme 2 gene $(L y z 2)$, abolishing endogenous $L y z 2$ function and placing NLS-Cre expression under the control of the Lyz2 promoter. 
The cre recombinase is expressed in cells of the myeloid lineage, including macrophages and neutrophils. The loxP excision efficacy of $L y s M^{\text {cre }}$ is highest in monocytes, mature macrophages and granulocytes. In microglia, loxP excision activity of $L y s M^{\text {cre }}$ has been shown to be up to $40 \%$ effective depending on CNS region ${ }^{32}$.

The fractalkine receptor, CX3CR1, is expressed in phagocytic cells comprising tissue resident macrophages, including microglia, and is also critical for blood derived monocyte survival. Accordingly, the expression of cre recombinase under the control of the $C x 3 c r l$ promoter, either as the constitutive $\left(C x 3 c r 1^{\text {cre }}\right)$ or tamoxifen inducible form $\left(C x 3 c r l^{\text {creER }}\right)$, mediates pan-macrophage recombination of the loxP site flanked allele ${ }^{33}$.

I generated $C x 3 c r 1^{\mathrm{cre}}-L R P 1^{\mathrm{fl} / \mathrm{fl}}$ and $C x 3 c r 1^{\mathrm{creER}}-L R P 1^{\mathrm{fl} / \mathrm{fl}}$ mice by crossing $L R P 1^{\mathrm{fl} / \mathrm{fl}}$ mice with $C x 3 c r 1^{\text {cre }}$ and $C x 3 c r 1^{\text {creER }}$ mice kindly provided by Dr. Steffen Jung. The $C x 3 c r 1^{\text {cre }}-L R P 1^{\mathrm{fl} / \mathrm{fl}}$ mice express cre recombinase under control of the fractalkine receptor promoter and has LRP1 constitutively deleted in both microglia and peripheral monocyte compartments. The $C x 3 c r 1^{\text {creER }}-L R P 1^{\mathrm{fl} / \mathrm{fl}}$ strain expresses cre recombinase under control of a tamoxifen inducible version of the fractalkine receptor promoter. Because CX3CR1 is expressed in microglia and monocytes, tamoxifen treatment initially leads to the deletion of LRP1 in microglia and peripheral monocyte compartments. However, the pool of circulating monocytes that can give rise to macrophages during inflammation is continuously renewed by progenitors from the bone marrow, while the long-lived brain resident microglia are not replenished and remain LRP1 deleted. As monocytes have a half-life of 3-4 days in the blood, they regain LRP1 expression four weeks after tamoxifen injection as the monocytes have been repopulated from the bone marrow. I also conducted studies in $L y s M^{\mathrm{cre}}-L R P 1^{\mathrm{fl} / \mathrm{fl}}$ mice, which have LRP1 deleted only in their 
peripheral myeloid population, from the Fazio lab at Vanderbilt University. In my studies I find that microglia from $L y s M^{\text {cre }}-L R P 1^{\mathrm{fl} / \mathrm{fl}}$ retain LRP1 expression, whereas peritoneal macrophages and bone marrow macrophages lack LRP1 expression.

\section{Role of Myeloid Cells in MS}

Among the immune cells involved in the pathogenesis of MS, myeloid cells are among the most abundant in the CNS during disease exacerbations. The myeloid cell population present during CNS inflammation includes macrophages derived from monocytes and microglia (Fig. 2). Monocyte-derived macrophages mature from peripheral monocytes infiltrating into the CNS during inflammation. The brain's resident macrophages, microglia, also contribute to the pool of myeloid cells involved in inflammation. Although macrophage depletion is associated with better disease outcome in animal models of $\mathrm{MS}^{34-38}$, it is still not fully understood how macrophages function in MS pathogenesis. Of particular importance, perivascular and meningeal macrophages have been shown to behave similarly to the resident microglia population of the brain, and current animal genetic tools do not allow for the distinction between these populations. We therefore consider them together with microglia for purposes of this work.

\section{Monocyte derived macrophages}

\section{Development and Homeostatic functions}

Blood-derived monocytes develop from hematopoietic stem cells in the bone marrow. Their differentiation relies on the presence of growth factor CSF-1 (M-CSF), 
normally present in the circulation and produced by various mesenchymal and epithelial cells $^{39}$. Monocytes originate from the common myeloid progenitor (CMP), which gives rise to the macrophage and dendritic cell progenitor population (MDP) that expresses fractalkine receptor CX3CR1 and stem cell growth factor receptor C-Kit.

Using an array of chemokine signals, including the MCP1/CCR2 and CSF-1 signaling axis, the monocytes enter the bloodstream and monitor for signs of infection or inflammation $^{40}$. Inflammatory monocytes will activate, proliferate, and migrate into organs based on the MCP1-CCR2 chemokine receptor system. The monocytes mature into macrophages to clear dead cells and secrete cytokines/chemokines to recruit other immune cells.

Monocytes/macrophages link inflammation and innate immunity against microorganisms to adaptive immune responses. They express high levels of pattern recognition receptors (PRRs), which include scavenger receptors and Toll-like Receptors (TLRs). These PRRs recognized danger associated molecular patterns (DAMPs) and pathogen associated molecular patterns (PAMPs), which include lipids, apoptotic cell components, and microorganisms, and play a critical role in activating the adaptive $\mathrm{T}$ cell response $^{41-43}$. Stimulation of PRR signaling pathways in monocytes/macrophages leads to nuclear translocation of a set of transcription factors, such as NF- $\kappa$ B and AP-1, initiating transcription of a plethora of inflammatory target genes. These genes transcribe seminal, pleotropic inflammatory effector molecules including TNF $\alpha$, Il-1 $\beta$, and IL- $6{ }^{43}$. These cytokines are responsible for altering vascular permeability, recruiting other blood cells to the inflamed tissue, and regulating the cell death in the tissue itself. Thus, there is tight transcriptional level control of these cytokines. 


\section{Pathological Role during CNS autoimmunity}

Infiltration of blood-derived monocytes and the presence of the peripherally derived macrophages in the CNS are required for $\mathrm{EAE}^{34,37,38,44}$. Deletion of CCR2 or administration of a CCR2 blocking antibody results in absence of monocyte/macrophage infiltration into inflamed tissue and protects against disease severity in MS mouse models $^{37,44-46}$. In the EAE lesion, macrophages are thought to contribute to disease pathogenesis by increasing inflammatory cytokine production, such as $\mathrm{TNF} \alpha^{47}$ and IL$1 \beta^{48}$, and initiating direct damage to the myelin at nodes of Ranvier ${ }^{49}$. Mice exhibit exacerbated EAE disease when NF- $\mathrm{KB}$ transcriptional activity is constitutively active in infiltrating myeloid cells ${ }^{50}$ and exhibit an ameliorated disease course when NF- $\mathrm{KB}$ activity is genetically ablated in infiltrating myeloid cells ${ }^{51}$. After the inflammatory insult subsides in EAE, the number of peripheral macrophages in the CNS decreases ${ }^{37}$. This is likely due to in situ apoptosis and drainage to lymph nodes, as was demonstrated for inflammatory macrophage fate in acute peritonitis ${ }^{52}$.

\section{Microglia}

Development and Homeostatic Functions

Aside from the peripherally derived macrophages, the CNS resident macrophages, microglia, have also been implicated in disease pathogenesis. Unlike infiltrating monocytes/macrophages, microglia are derived from primitive macrophage populations in the fetal yolk sac and require no further contribution from the periphery during development. Only recently has it been shown that microglia are self-renewing and are 
not replenished by circulating monocytes physiologically, although both express the fractalkine receptor $(\mathrm{CX} 3 \mathrm{CR} 1)$ required for leukocyte adhesion and tissue invasion during inflammation ${ }^{53-55}$.

Microglia are essential for sustaining CNS function by pruning neurons in an activity-dependent fashion and clearing apoptotic cells in development and homeostasis 56-59. Microglia constantly survey the CNS environment through dynamic movement of their processes to maintain systemic homeostasis. In vivo imaging shows rapid microglial activation in response to CNS damage induced by laser ablation and presence of DAMPs (ATP) and PAMPs (LPS) administered to the $\mathrm{CNS}^{60,61}$

Pathological role during CNS autoimmunity

Despite their important physiological role, several lines of evidence suggest that microglial activity is detrimental in EAE. The activation of microglia can be detected before onset of disease in EAE and their activation also contributes to EAE clinical outcome $^{62,63}$. First, microglial activation and proliferation can be detected via flow cytometry well before the onset of clinical symptoms in EAE. Second, microglial ablation of NF- $\kappa \mathrm{B}$ upstream kinase TAK1 prevents EAE onset ${ }^{37}$, illustrating the pivotal role of $\mathrm{NF}-\kappa \mathrm{B}$ mediated inflammation in the brain's resident phagocytes in MS disease initiation. Although these studies suggest that microglial activity contributes to EAE progression, other studies suggest that microglia play an important role in restoring normal CNS physiology by clearing of phagocytic debris during resolution of an inflammatory episode $^{49}$. Third, directly inducing "microglial" death in transgenic CD11b-HSV-TK mice, irradiation and reconstitution with wild type bone marrow causes less clinically severe $\mathrm{EAE}^{63}$. However, Heppner et al. did not consider that although they observe 
microglial death, other $\mathrm{CD} 11 \mathrm{~b}+$ populations, including the peripheral monocyte-derived macrophages, would also be susceptible to death. Thus, this study actually illustrates that targeting proliferation of the $\mathrm{CD} 11 \mathrm{~b}+$ myeloid cell populations, which include the microglia and the peripherally derived macrophages, is beneficial during EAE.

Historically it has been difficult to differentiate between these two myeloid populations, as there is no distinguishing histological protein marker. Even current microglia-specific markers discovered such as P2ryl2 and Fcrls change expression with regards to inflammation, which make them difficult to use during CNS inflammation such as $\mathrm{EAE}^{64,65}$. But by using specific cre-recombinase-driven reporter mice and techniques such as bone marrow transfer, researchers have been able to differentiate between the two populations with findings that illustrate the distinct and discrete roles of microglia and macrophages during EAE pathogenesis.

Using parabiosis, Ajami et al. showed that population of microglia increases in the brain during EAE before the accumulation of peripheral macrophages ${ }^{37}$. Furthermore, Yamasaki et al. used electron microscopy to describe the function of monocyte-derived macrophages as active destroyers of myelin, while microglia are protective phagocytic debris cleaners ${ }^{49}$. Thus it is intriguing to consider if the expression and activity of the receptor instrumental to myeloid cell function would differ on these two cell types in the context of disease, and how that correlates to their physiological function. Understanding the role of the different microglia/macrophage populations in EAE would also benefit our understanding of the initiation, progression and resolution of inflammation in MS. 


\section{Molecular mediators of myeloid inflammation during MS: NF-кB}

As innate immune cells, myeloid cells are responsible for maintaining and propagating inflammation when the organism comes under perceived attack. The canonical model of macrophage inflammatory activation describes a classical and alternative activation status. M1 classical macrophage activation, defined by treatment with either LPS or IFN- $\gamma$, skews macrophages toward a more proinflammatory phenotype. These macrophages produce increased reactive oxygen species and inflammatory cytokines, such as IL-6 and TNF $\alpha$. M2 alternative macrophage activation, defined by treatment with IL-4, skews macrophages toward a more anti-inflammatory phenotype. Macrophages secrete increased amounts of anti-inflammatory cytokines, such as IL-10 and TGF $\beta$. These categories initially arose only to describe the behavior of macrophages in a dish when treated with the aforementioned cytokines. Now, researchers are finding that the behavior and expression markers of macrophages in vivo is complex beyond the scope of these descriptors, with findings that macrophages may co-express markers traditionally associated with both the M1 and M2 phenotype during disease pathogenesis $^{66}$. Nonetheless, the M1/M2 dichotomy of macrophages is a useful categorization of general inflammatory response behavior of macrophages in vitro.

An acute inflammatory response concomitant with active demyelination is thought to be responsible for MS attacks. During the neuroinflammatory process in MS, rampant cell death and inflammation causes release of DAMPs and PAMPs. These ligands bind to conserved pattern recognition receptors, such as Toll-like Receptors (TLRs), on myeloid and other innate immune cells to activate downstream inflammatory 
pathways $^{67}$. One of these is the NF- $\mathrm{B}$ inflammatory transcriptional pathway, which regulates expression of cytokines such as TNF $\alpha$, IL- 6 and IL-1 $\beta^{68-71}$ to propagate the inflammatory response by further activating other immune cells and increasing phagocytic activity $^{39-42}$. In human MS lesions, immunohistochemical studies revealed increased activated NF- $\kappa \mathrm{B}$ reactivity, especially in myeloid cells ${ }^{72,73}$

\section{NF-кB: signaling pathways}

First described by Sen and Baltimore over twenty years ago, the NF- $\kappa$ B pathway has emerged as a key transcriptional regulator of the inflammatory response ${ }^{74}$. The NF$\kappa \mathrm{B}$ response can be classified into two pathways, the canonical and non-canonical pathway. As the non-canonical pathway is primarily involved in the development of lymphoid tissues, I will focus on the discussion on the canonical pathway.

The NF- $\kappa \mathrm{B}$ family of transcription factors is composed of two subfamilies. The

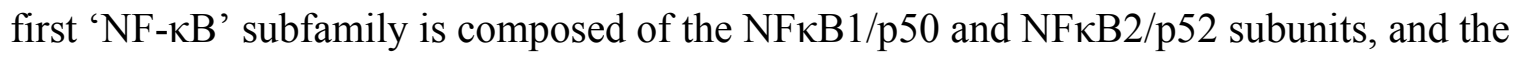
second 'Rel' subfamily consists of the RelA/p65, c-Rel, and RelB subunits. All of the proteins contain a conserved DNA binding domain called the Rel homology domain (RHD), and the Rel subfamily contains C-terminal transactivation domain. These subunits can form either homo or heterodimers, and the most abundant dimer is the p50/p65 heterodimer ${ }^{75}$.

$\mathrm{NF}-\kappa \mathrm{B}$ dimers are constitutively expressed and bound to a repressor I $\kappa \mathrm{B}$ protein

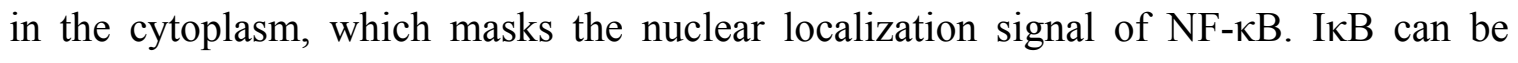
phosphorylated by the I $\mathrm{B}$ kinase (IKK) complex, composed of three subunits; Ikk $\alpha$, Ikk $\beta$, and Ikk $\gamma / \mathrm{NEMO}$, which is the regulatory subunit. Inflammatory signaling such as 
TLR activation causes downstream activation of the IKK complex, which then phosphorylates $\mathrm{I} \kappa \mathrm{B}$ and causes its ubiquitination and degradation by the proteasome ${ }^{76}$. The NF- $\mathrm{BB}$ dimer is then free to translate to the nucleus and induce target gene transcription. A schematic of NF- $\mathrm{BB}$ signaling downstream of TLR signaling, as well as our working hypothesis regarding the role of LRP1 in regulating this signaling, is found in Figure 3.

The NF- $\mathrm{B}$ pathway regulates innate and adaptive immune responses. Numerous studies show that NF- $\mathrm{B}$ contributes to MS progression. Increased NF- $\kappa \mathrm{B}$ activity is correlated with CNS disease severity ${ }^{77}$. In EAE, there is also increased NF- $\kappa \mathrm{B}$ transcriptional activity in the mouse spinal $\operatorname{cord}^{78}$. Furthermore, NF- $\mathrm{Bp} 50^{-/-}$mice are resistant to $\mathrm{EAE}^{79}$, and mice deficient in upstream kinases of the $\mathrm{NF}-\kappa \mathrm{B}$ signaling cascade IKK $\beta$ or IKK $\gamma / \mathrm{NEMO}$, are also resistant to $\mathrm{EAE}^{80}$. When there is constitutive activity of the inflammatory transcription factor NF- $\kappa \mathrm{B}$ pathway in infiltrating myeloid cells, the mice have exacerbated EAE disease ${ }^{50}$. Deleting the NF- $\kappa \mathrm{B}$ regulating IKK $\beta$ subunit in the peripheral myeloid compartment under control of the $L y s M^{\text {Cre }}$ promoter protects mice against EAE severity ${ }^{51}$. Thus NF- $\kappa \mathrm{B}$ has been suggested as a promising therapeutic target for MS.

$\mathrm{NF}-\kappa \mathrm{B}$ governs an important inflammatory transcriptional response in immunity, but is also an important transcription factor involved in numerous physiological functions such as apoptosis and growth. Targeting NF- $\kappa \mathrm{B}$ in MS has therefore been focused on products of inflammatory $\mathrm{NF}-\kappa \mathrm{B}$ signaling, such as $\mathrm{TNF} \alpha$. Autopsy samples of MS patients show TNF $\alpha$ staining associated with astrocytes and macrophages in the lesion site $^{81}$. Higher levels of TNF $\alpha$ in patient cerebrospinal fluid (CSF) is also correlated to 
disease severity and increased neurologic disability and deterioration ${ }^{82}$. Anti-TNF $\alpha$ prevented EAE development, and so was hailed as a potentially putative therapeutic for $\mathrm{MS}^{83}$. Therefore, it was of great surprise to the field when MS patients who enrolled in a clinical trial for $\mathrm{TNF} \alpha$ receptor fusion protein experienced increased and more severe exacerbations of disease, causing the early termination of the trial ${ }^{84}$. Further studies suggest that this surprising result may be due to the differential effects of signaling through TNFR1 vs. TNFR2 during disease progression; the former appears to promote the proinflammatory effects of MS, while the latter appears to be important for the termination of inflammation ${ }^{85,86}$. Current murine studies now attempt to address the role of TNF $\alpha$ at different stages of disease by varying time of treatment ${ }^{87}$. In terms of antibody treatment, administration of $\mathrm{TNF} \alpha$ antibody to patients with secondary progressive MS had no effect ${ }^{88}$. The failure of treatments targeting TNF $\alpha$ points to an urgent need to not only address the clinically robust symptoms of inflammation correlated with increased TNF $\alpha$ levels, but to also understand the mechanisms of these molecules at the cellular level. In particular, cellular processes that modulate inflammation could be extremely beneficial towards understanding alternative mechanisms for targeting immune activity during MS.

\section{The crosstalk between phagocytosis and inflammation}

During MS attacks, in addition to the rampant inflammation induced by the immune system, there are copious amounts of myelin and apoptotic cell debris produced. Although phagocytic activity is increased following inflammatory cues, the internalization of this debris appears anti-inflammatory. The professional phagocytes 
found in the MS lesion, the peripherally derived macrophages and CNS resident microglia, are also active producers of $\mathrm{NF}-\kappa \mathrm{B}$ regulated inflammatory cytokines such as TNF $\alpha$. Phagocytosis seems to be implicated in the resolution of inflammation, and this will be discussed in the next section.

A central function of myeloid cells in the MS lesion is to efficiently initiate phagocytosis, defined as the cellular engulfment of large particles $(>0.5 \mu \mathrm{m}){ }^{89}$. This function is initiated by the recognition of microbial epitopes or apoptotic/necrotic cell material by PRRs that are abundantly expressed on microglia and macrophages. Although dependent on the binding receptor, the phagocytic process can be broadly outlined as follows ${ }^{90}$ :

a) Receptor-mediated binding of extracellular particles to cell surface receptors, such as LRP1;

b) Clathrin-mediated endocytosis of the receptor-bound particle(s),

c) phagosome maturation and increasing acidification in the mature phagolysosome,

d) destruction of the receptor-bound particle by digestive enzymes in the lysosome or resecretion of the ligands from recycling endosomes or trafficking of the ligand from apical to the basolateral membrane ${ }^{91}$, and

e) recycling of the receptor back to the cell surface

Lysosomal activity is essential for the regulation of degradation of particles and recycling of phagocytic receptors back to the cell surface. Increased lysosomal activity leads to translocation of the transcription factor EB (TFEB) into the nucleus, where it increases lysosomal biogenesis and promotes further degradation of complex molecules. 
Various genes are also upregulated in response to TFEB overexpression, including chloride channel 7 (CLCN7), ATPase $\mathrm{H}+$ Transporting V1 Subunit $\mathrm{H}(A T P 6 V 1 H)$, Cathepsin A (CTSA), Hexosaminidase Subunit Alpha (HEXA), and Lysosomalassociated membrane protein $1(\operatorname{Lamp} 1)^{92}$. Therefore, monitoring the expression of these genes is useful for detecting perturbations in the phagocytic activity.

\section{Phagocytosis in MS: a restorative, anti-inflammatory mechanism}

During MS attacks, there are copious amounts of myelin debris produced. Myelin debris clearance is crucial for regeneration and repair of neurons, as well as regeneration of healthy myelin ${ }^{93}$. Presence of myelin debris in the CNS arrests OPC differentiation ${ }^{94}$. In the cuprizone remyelination model, it has been shown that mice lacking the fractalkine receptor CX3CR1 lose ability to clear myelin debris, preventing proper remyelination ${ }^{95}$. The literature suggests that phagocytosis of myelin debris by myeloid cells decreases the inflammatory phenotype of macrophages derived from human MS patients, as myelinladen macrophages showed decreased cytokine production in response to LPS stimulation compared to non-myelin treated macrophages ${ }^{96}$. Within the MS lesion, foamy myelin laden macrophages also have an anti-inflammatory phenotype as they show increased expression of IL-1ra, IL-10, and TGF ${ }^{96}$. This suggests that myelin phagocytosis inhibits inflammation through a negative feedback mechanism.

Furthermore, apoptotic disintegration of cells in situ and release of intracellular components is known to exacerbate local inflammatory responses based on recognition by PRRs such as TLRs. However, in vitro studies on human PBMC derived macrophages demonstrate that ingestion of apoptotic cells actually promotes an anti-inflammatory and 
immune-suppressive effect ${ }^{97}$. In LPS treated cultures of human macrophages, pretreatment of apoptotic cells resulted in decreased protein expression of TNF $\alpha$, IL-1 $\beta$ and Il-12 levels ${ }^{98}$. In vivo administration of LPS with injection of apoptotic Jurkat cells also decreases local tissue inflammatory cytokine levels and increases production of antiinflammatory TGF- $\beta$ in a murine model $^{99}$. Opsonization of the apoptotic cells reverses the anti-inflammatory effect observed. During MS, numerous T cells and myeloid cells infiltrate into the CNS; it has been shown that ingestion of apoptotic $\mathrm{T}$ cells and peripheral myeloid cells promotes an anti-inflammatory program in microglia ${ }^{100,101}$. Thus, phagocytosis is also recognized as an essential effector of inflammation resolution.

\section{Toll like receptors in phagocytosis}

Myeloid cells are the professional phagocytes in the body. They express a variety of receptors that recognize and internalize debris during homeostasis and disease. One of these receptor families is the Toll like receptors (TLRs). This group of receptors, highly expressed on the surface of innate immune cells, binds to DAMPs and PAMPs and signal through downstream adaptor protein signaling pathways to initiate transcriptional pathways NF- $\kappa$ B. TLRs preferentially use either the MyD88 or the TRIF dependent adaptor protein pathways, and the TLR-mediated induction of scavenger receptors/phagocytosis has been shown to be MyD88 dependent ${ }^{102}$. TLR4 is the only receptor that uses both the MyD88 and TRIF dependent adaptor protein pathways. TLR3 exclusively uses the TRIF dependent pathway, while the other TLRs exclusively use the MyD88 dependent pathway. 
TLR stimulation and subsequent activation of microglia results in increased cytokine and chemokine production, as well as proliferation ${ }^{103,}{ }^{104}$. Two photon microscopy reveals that in vivo injection of LPS (TLR4 ligand) also results in increased activity of microglial processes ${ }^{60}$. In vivo administration of A $\beta$ (TLR2 ligand) has been shown to increase microglial phagocytic activity and neuronal loss, while blocking phagocytosis seems to prevent neuronal death ${ }^{105}$. TLR stimulation also increases phagocytic activity in macrophages through appropriating cellular structures involved in the autophagosome pathway ${ }^{106}$.

\section{Scavenger receptors in phagocytosis: TREM2}

Besides TLRs, scavenger receptors are also robust regulators of the inflammatory response, and also of debris clearance, expressed on myeloid cells. In 2012, 2 independent groups identified several heterozygous variants of the Triggering receptor expressed on myeloid cells 2 (TREM2) gene that increases susceptibility to Alzheimer's Disease $(A D)$ with an odds ratio similar to that of Apo\&4 ${ }^{107,108}$. TREM2 is a scavenger receptor expressed at the cell surface of myeloid cells, including macrophages and microglia, and negatively regulates inflammatory and TLR responses by pairing with downstream adaptor protein DAP12 $2^{109,110}$. TREM2 also mediates clearance of apoptotic neurons and bacteria ${ }^{111,112}$.

There is an abundance of plaque associated myeloid cells found in the AD mouse model, and they express high levels of TREM2. The anti-inflammatory and phagocytic ability of TREM2 suggests that mice should have exacerbated disease when TREM2 is 
deleted $^{113}$. However, researchers have found that both Trem 2 deficiency, and overexpression, is protective against disease pathogenesis in AD mouse models ${ }^{111,114,115}$.

As TREM2 is also a receptor for myelin phagocytosis, there is also increasing interest in the role of this receptor in $\mathrm{MS}^{116}$. Early investigations have found that a cleaved form of soluble TREM2 is found at high levels in CSF of MS patients ${ }^{117}$. $T R E M 2^{-/}$mice have defective clearance of myelin debris and more axonal pathology in a mouse model of $\mathrm{MS}^{116}$, and administering TREM2 blocking antibody exacerbates EAE disease progression ${ }^{118}$.

Therefore, scavenger receptors, especially those that bind apoptotic cells and myelin, are of great interest to researchers in the context of neuroinflammatory and neurodegenerative diseases.

\section{LRP1: a key regulator of inflammation and phagocytosis}

Scavenger receptors are essential to the recognition and engulfment of the cell debris in MS. As previously discussed, inflammation and phagocytosis are intrinsic functions of myeloid cells during MS pathology progression. Our laboratory has studied the function of a receptor, low-density lipoprotein receptor 1 (LRP1), which I show is involved in regulation of inflammation and phagocytosis.

\section{Structure}

LRP1 is a member of the LDL receptor superfamily, which includes very lowdensity lipoprotein (VLDL) and LDL receptor. Unlike deletion of Vldlr and Ldlr, however, deletion of the Lrpl gene is embryonic-lethal in mice ${ }^{119}$. LRP1 is a cell surface apolipoprotein receptor known to recognize, endocytose, and regulate the signaling 
pathways of a multitude of ligands, ranging from surface receptors to matrix metalloproteinases ${ }^{120}$. LRP1 transcription is regulated by a sterol regulatory element (SRE) binding sequence and expression of the transcript is responsive to hormones and growth factors ${ }^{121}$ In macrophages, transcription of LRP1 is increased by CSF-1 and downregulated by IFN $\gamma$ and $\operatorname{LPS}^{122}$. Synthesized as a $600 \mathrm{kD}$ protein, LRP1 is cleaved in the trans Golgi network by furin into an extracellular $515 \mathrm{kD}$ alpha chain and transmembrane $85 \mathrm{kD}$ beta chain ${ }^{123}$. The beta chain also contains multiple protein domains which are involved in tyrosine and serine phosphorylation, endocytosis signaling, protein maturation, and internalization of the receptor ${ }^{124}$. The alpha and beta chains are non-covalently bound at the cell surface. Cleavage of LRP1 at the cell surface by ADAM17 and subsequent production of a truncated, soluble form of LRP1 (sLRP1) can be induced by inflammatory LPS and IFN $\gamma$ signaling ${ }^{125}$.

\section{LRP1 Function: Phagocytosis}

LRP1 was first characterized as an apolipoprotein E receptor, responsible for the recognition and internalization of ApoE on the cell surface. However, further studies have revealed that it is a promiscuous receptor which binds to over 100 identified ligands, including tissue transglutaminases, tPA, thrombospondin, ApoE, $\mathrm{A} \beta$ peptide, and $\mathrm{C} 1 \mathrm{q}^{126-}$ ${ }^{128}$. Thus, it has since been classified as a scavenger receptor. Examples of LRP1 ligands are presented in Table 1.

A multitude of downstream signaling pathways are affected by the phagocytic activity of LRP1 due to the diversity of its ligands. For instance, PDGF $\beta$ receptor signaling leads to the phosphorylation of LRP1, which then acts as a competitor for SHP- 
2 binding downstream of PDGF signaling ${ }^{129}$. LRP1 also associates with PDGF receptor in the endosome, and is necessary for PDGF mediated ERK signaling ${ }^{130}$.

Our lab was the first to show that LRP1 is also involved in myelin phagocytosis, and we identified specific LRP1 ligands, myelin basic protein and myelin-associated glycoprotein, within myelin ${ }^{131,132}$. Using mass spectrometry, we also discovered an array of intracellular proteins that are recognized by LRP1, pointing to a function in the removal of necrotic cell debris ${ }^{133}$.

\section{LRP1 Function: Inflammation}

In addition to its role in phagocytosis, our lab previously showed that LRP1 is a regulator of the $\mathrm{NF}-\kappa \mathrm{B}$ signaling pathway mediated inflammatory response. LRP1 deficient fibroblasts have increased levels of NF- $\mathrm{BB}$ binding activity, as well as downstream activation of NF- $\kappa \mathrm{B}$ transcriptional targets such as IL-6 and inducible nitric oxide synthase (iNOS) ${ }^{134}$. Overton et al. demonstrated that LRP1 dampens the inflammatory response by suppressing expression of mediators such as MCP-1 (CCL2), TNF $\alpha$, and MMP9 in thioglycollate-derived peritoneal murine macrophages ${ }^{135}$.

In the peripheral nervous system, injured Schwann cells increase expression of LRP1 and shed a soluble form of LRP1 (sLRP1) with an intact alpha chain. Injecting this purified sLRP1 into sciatic nerves prior to injury decreases expression of TNF $\alpha$ and IL$1 \beta^{136}$. In inflammatory autoimmune conditions such as rheumatoid arthritis and systemic lupus erythematosis, patients also have increased amount of sLRP1 in their circulation ${ }^{125}$.

In a sepsis model of LPS injection, peripheral macrophage LRP1 deficient animals showed decreased survival rates and corresponding increased systemic amounts of circulating TNF $\alpha$ and CCL3 ${ }^{137}$. There was also increased IL-6 and iNOS mRNA 
expressed in the organs of these mice. Inhibiting LRP1 through pharmacological inhibition with its antagonist, RAP, also increased the production of proinflammatory cytokines in bone marrow macrophages ${ }^{137}$. Independently, LRP1-deficient BMDM showed increased cellular homing and migration, as they secrete increased levels of CCL2 and CCL3 in response to LPS stimulation, and LRP1-deficient monocytes also increase migratory activity in a model of pancreatic cancer ${ }^{138}$. When cultured with low FCS medium, LRP1-deficient BMDM also downregulate anti-inflammatory markers Arg1, SOCS3, and IL-1RA ${ }^{139}$. LRP1 has also been shown to downregulate cell surface TNFR1, which possibly contributes to its anti-inflammatory function ${ }^{134}$. LRP1 is also implicated in other axis of TNF $\alpha$ signaling. Deleting LRP1 in macrophages abrogates the anti-atherogenic effect of administering anti-TNF $\alpha$ to mice, suggesting that LRP1 is involved in the atheroprotective effect of antagonizing TNF $\alpha$ activity ${ }^{140}$.

Zurhove et al. proposed that LRP1's anti-inflammatory effect is based on an IRF3 dependent mechanism. They show that with LPS stimulation, LRP1 is cleaved by $\gamma$ secretase and the intracellular, cleaved beta chain enters the nucleus. The beta chain prevents NF- $\kappa \mathrm{B}$ binding to the IFN $\gamma$ promoter and also mediates IRF3 export from the nucleus to downregulate transcription of IRF3-dependent cytokines ${ }^{141}$. Other groups have shown that expression of LRP1 promotes expression of genes linked to the M2 phenotype independently of IL-4 stimulation, and macrophages express higher levels of LRP1 when skewed to the M2 phenotype ${ }^{142,143}$. Antagonizing LRP1 activity through administration of its antagonist RAP or lactoferrin was also shown to increase NF-kB activity within 5 minutes of treatment. This inflammatory activity was linked to the upregulation of 
microRNA miR-155 ${ }^{144}$. Thus, there are many lines of evidence across different disease models suggesting that LRP1 plays an important role in the regulation of inflammation.

\section{LRP1 in neurodegeneration: findings in MS}

Due to its versatile functions and involvement in the critical lipoprotein transport cycle, LRP1 is expressed at a basal level throughout the body. Within the immune system, LRP1 is most highly expressed on myeloid cells, which include macrophages and microglia ${ }^{145}$ (Fig. 4a). In the CNS, LRP1 transcript is also expressed on multiple cell types, including neurons and endothelial cells (Fig. 4b) ${ }^{146}$. Recent studies reveal that LRP1 is expressed highly on microglia at the RNA level at baseline and during EAE (Fig. $4 \mathrm{c}-\mathrm{d})^{65,147}$. LRP1 has been implicated in a number of neurodegenerative diseases. Ever since its discovery as an $\mathrm{A} \beta$ receptor, there has been increasing interest in LRP1 in Alzheimer's research ${ }^{148,}{ }^{149}$, especially with evidence that endothelial cell LRP1 transports $\mathrm{A} \beta$ across the blood brain barrier $(\mathrm{BBB}){ }^{150}$. LRP1 expression on pericytes has also been implicated in the integrity of the $\mathrm{BBB}^{151}$.

In the context of MS, failure to clear products of myelin degeneration and apoptotic cell debris may contribute to initiating the immune response ${ }^{152}$. As LRP1 recognizes and clears apoptotic cells and myelin debris, found in abundance in MS lesions, the activity of LRP1 may be involved in the disease pathogenesis of $\mathrm{MS}^{131,133}$. Furthermore, LRP1 expression is upregulated in areas of demyelinated MS lesions by qPCR analysis ${ }^{153}$. By mRNA expression analysis, LRP1 expression on microglia has been shown to be 1000 -fold that of peripheral blood monocytes at baseline ${ }^{65}$. During the development of EAE, LRP1 expression on monocytes increases to nearly the level of 
expression found in microglia ${ }^{147}$. This elevated level of LRP1 expression in myeloid cells, especially microglia, suggests that LRP1 may play an important role in microglia function in homeostasis and during autoimmune disease.

Intriguingly, microglial phagocytosis of apoptotic inflammatory $\mathrm{T}$ cells was shown to decrease levels of microglia activation ${ }^{101}$. This has resounding implications for MS, as there is an abundance of apoptotic and necrotic infiltrating immune cells, including $\mathrm{T}$ cells. Based on these findings, I postulated that LRP1's role as a putative anti-inflammatory protein may be causing its upregulation in the active, inflammatory lesion in efforts to provide negative feedback in response to inflammatory cues (Fig. 5).

\section{Rationale and hypothesis}

MS is a disease with a heavy quality of life burden and affects global populations. Since patients are usually diagnosed between 20-30 years of age, the disease has a large impact on their adult working life. The disease etiology remains unidentified, but we do know that the disease course can be modified by immune targeting treatments. Many of these drugs work to decrease the inflammatory responses, especially of $\mathrm{T}$ cells, in the CNS. Additional studies of the inflammatory response mediated by different populations of immune cells during MS disease pathogenesis will prove useful both in understanding the biological mechanisms of MS as well as paving the way to developing different treatments. Thus, I focus on the contributions of different myeloid cell populations to the inflammatory response in MS.

Aside from T cells, myeloid cells are the most numerous immune cell type in the MS lesion. Myeloid cells contribute to disease progression in several ways. First, they are 
producers of inflammatory mediators such as TNF $\alpha$, IL-1 $\beta$, and IL-6, which are linked to disease exacerbations in MS. Second, they are also professional phagocytes involved in the clearance of the myelin and apoptotic debris in the MS lesion. These functions both contribute to disease progression in MS, but the potential of crosstalk between phagocytosis and inflammation is still largely unexplored.

The CD68+ myeloid cells in MS come from two different origins: the CNS resident microglia and the peripheral monocyte-derived macrophages. The literature on the contributions of these populations to disease pathogenesis remains controversial. In EAE, microglia have been shown to be relatively immune silent compared to peripheral macrophages, but ablation of the inflammatory NF- $\mathrm{kB}$ transcriptional response, through deleting an upstream kinase, protects mice from EAE. Simultaneously, infiltration of the monocyte-derived macrophages is essential for disease pathogenesis. Mice exhibit exacerbated EAE disease when NF- $\mathrm{KB}$ transcriptional activity is constitutively active in infiltrating macrophages and exhibit an ameliorated disease course when NF- $\mathrm{kB}$ activity is genetically ablated in infiltrating macrophages ${ }^{50,51}$. Therefore, understanding their precise contributions to the inflammatory events is useful for exploring ways in which inflammation may be further regulated by pharmacological methods during EAE, and perhaps MS.

By immunohistochemistry alone, there is no unique expression marker that can differentiate these two populations. Therefore, I turn to using mouse models that can target genes in either population using specific promoter-driven cre recombinase. My target gene of choice is LRP1, an apolipoprotein receptor involved in both phagocytosis and inflammation. LRP1 expression on glial cells is increased in CNS injury and 
neoplasia, and its expression has been shown to regulate NF-kB transcription. Thus I hypothesized that deleting LRP1 in myeloid cells, in either microglia or peripheral macrophages, may cause uninhibited NF- $\mathrm{kB}$ activity, causing exacerbation of disease in EAE. I further sought to explore whether deletion of LRP1 could decrease phagocytic activity of these myeloid cells. 


\section{Figures}

Figure 1: History of MS and Current Therapies

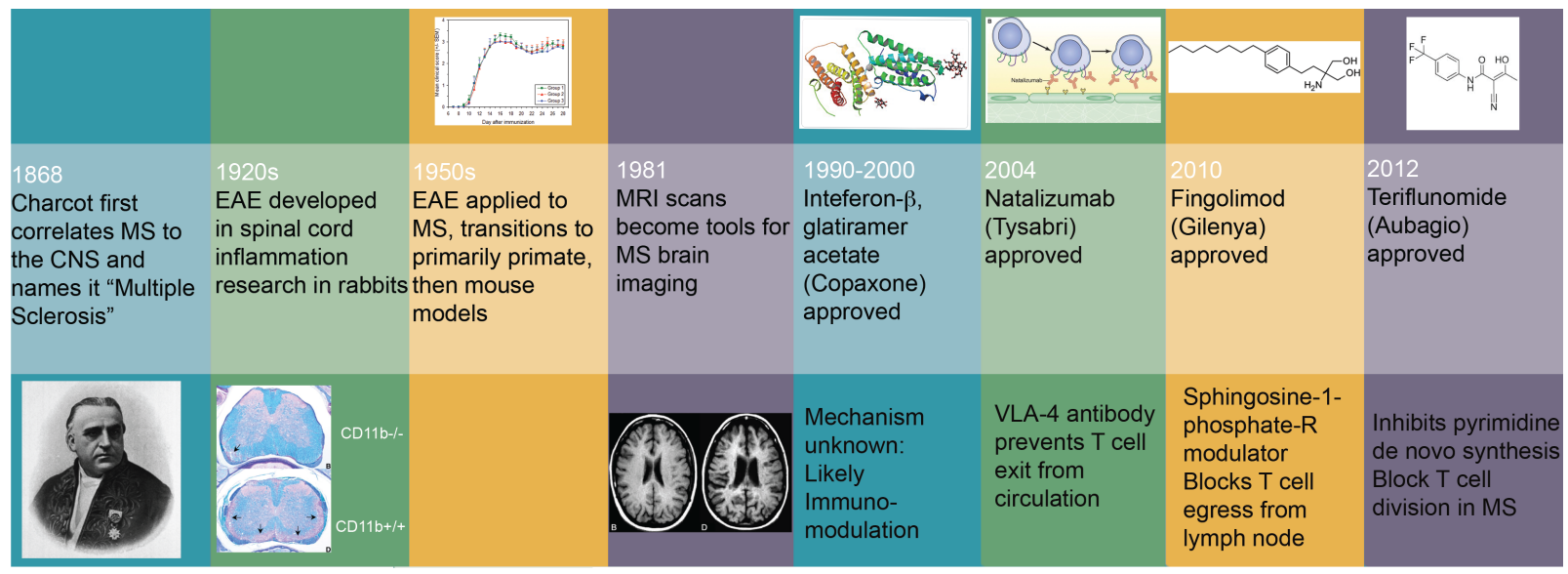

The timeline outlines the discovery of MS, EAE, and some of the disease modifying drugs available today for MS treatment. 
Figure 2: Genetic tools for interrogating LRP1 function on myeloid cells of different origin during EAE

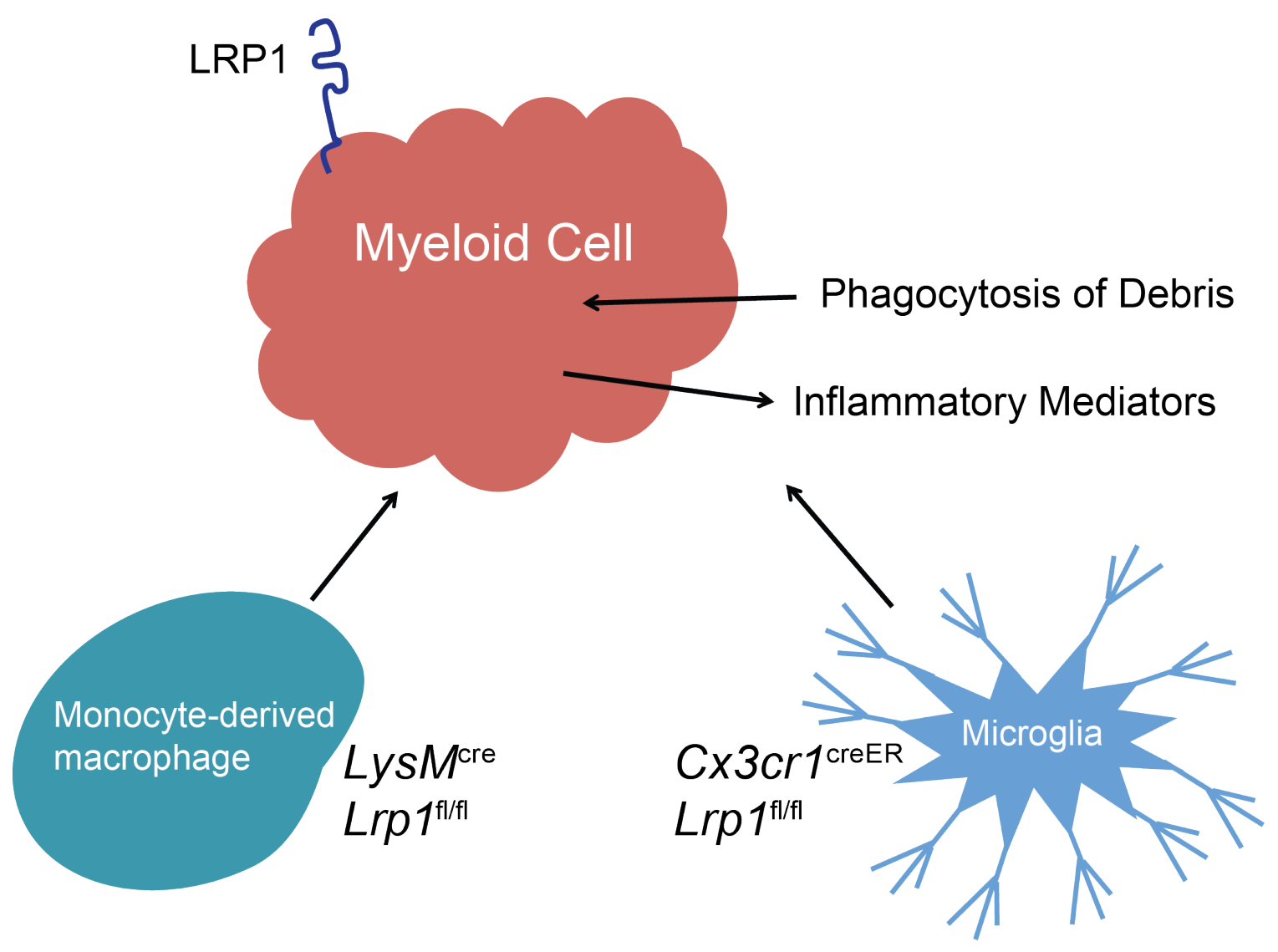

Myeloid cells express high levels of LRP1. During EAE, these cells originate from the monocyte-derived macrophages, whose LRP1 expression can be targeted in $L y s M^{\text {cre }}$ $L R P 1^{\mathrm{fl} / \mathrm{fl}}$ mice, or microglia, whose LRP1 expression can be targeted in $C x 3 \mathrm{cr} 1^{\text {creER }}$ $L R P 1^{\mathrm{fl} / \mathrm{fl}}$ mice. 
Figure 3: TLR/NF-xB signaling schematic

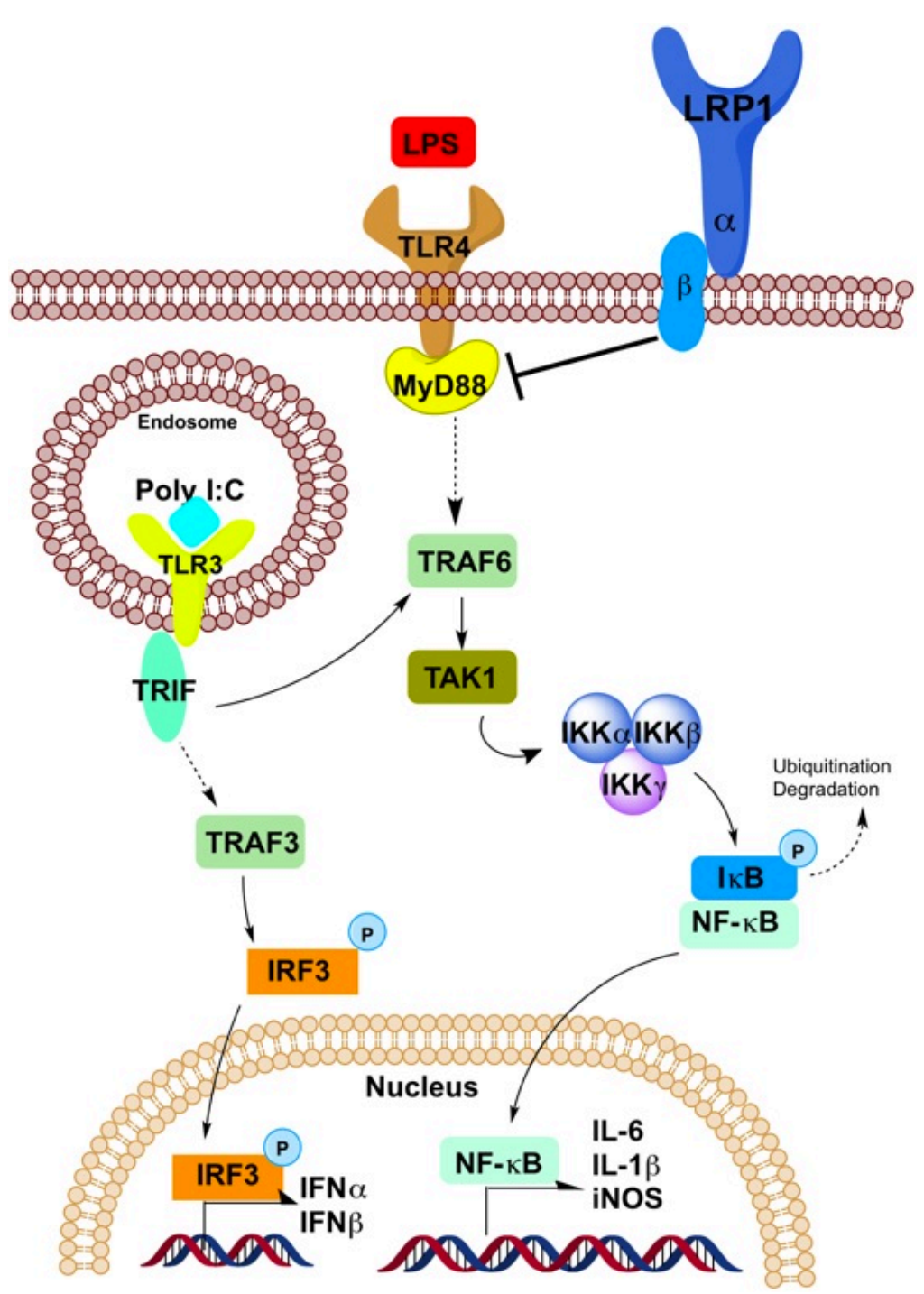

Signaling downstream of TLR activation is mediated by either the MyD88 or TRIF dependent signaling pathways. TLR4 uses both the TRIF and MyD88 dependent pathways, TLR3 exclusively uses the TRIF dependent pathway, and all other TLRs exclusively use the MyD88 dependent pathway. NF- $\mathrm{kB}$ is activated downstream of TLR signaling. In this work I show that LRP1 is involved in regulating TLR signaling by inhibiting the MyD88 dependent pathway. 
Figure 4: Examining LRP1 expression by mRNA analysis

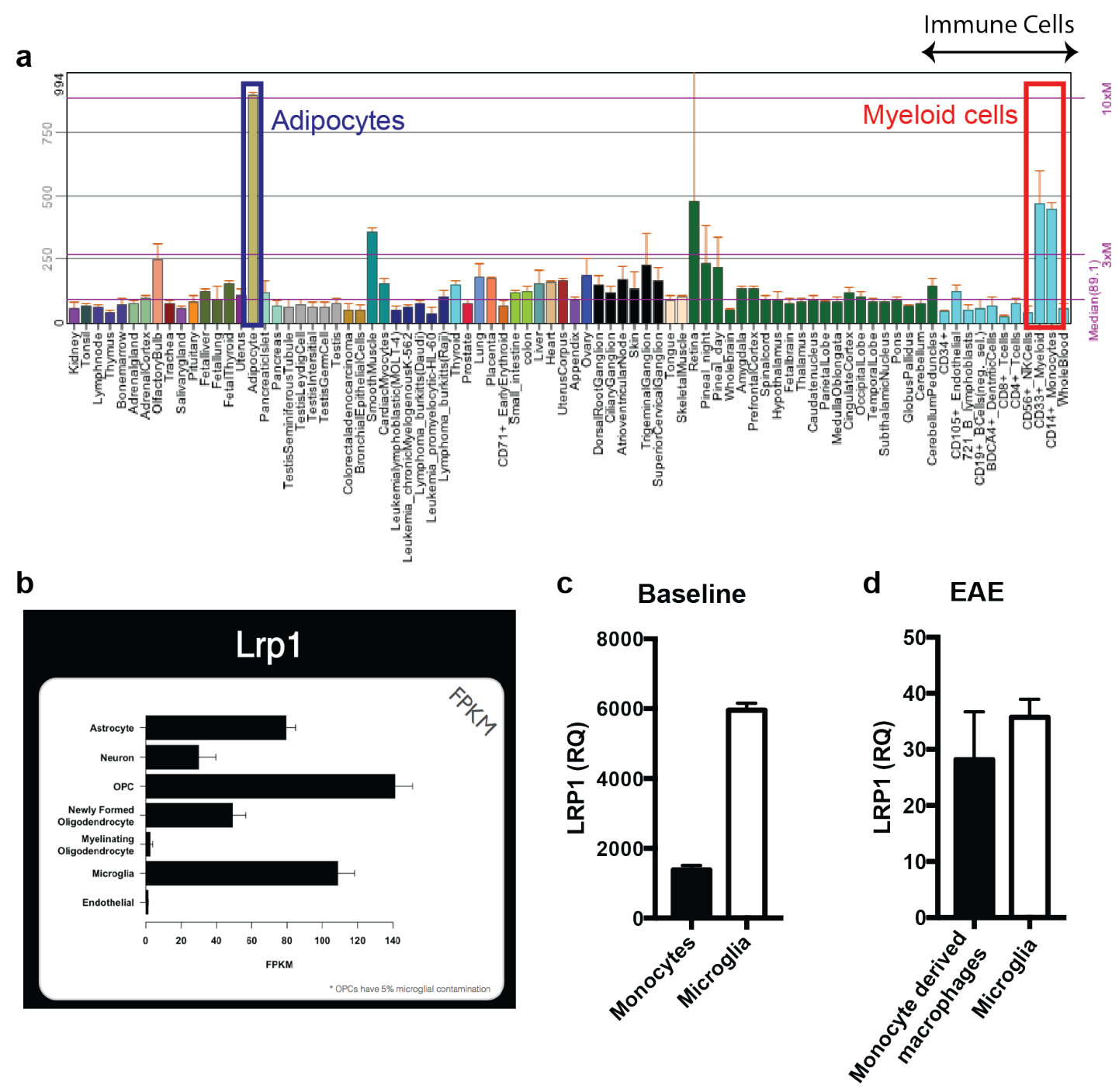

a LRP1 expression in different organs and tissues as analyzed by the human genome atlas $(\mathrm{GNF})^{154}$. LRP1 is the most highly expressed on adipocytes, and within the immune cell subset, on myeloid cells. b LRP1 expression in isolated CNS cell types ${ }^{146}$. c LRP1 expression by qPCR analysis on monocytes and microglia from wild type mice ${ }^{65}$. d LRP1 expression on monocytes and microglia during EAE ${ }^{147}$. 
Figure 5: LRP1 at the crossroads of phagocytosis and inflammation

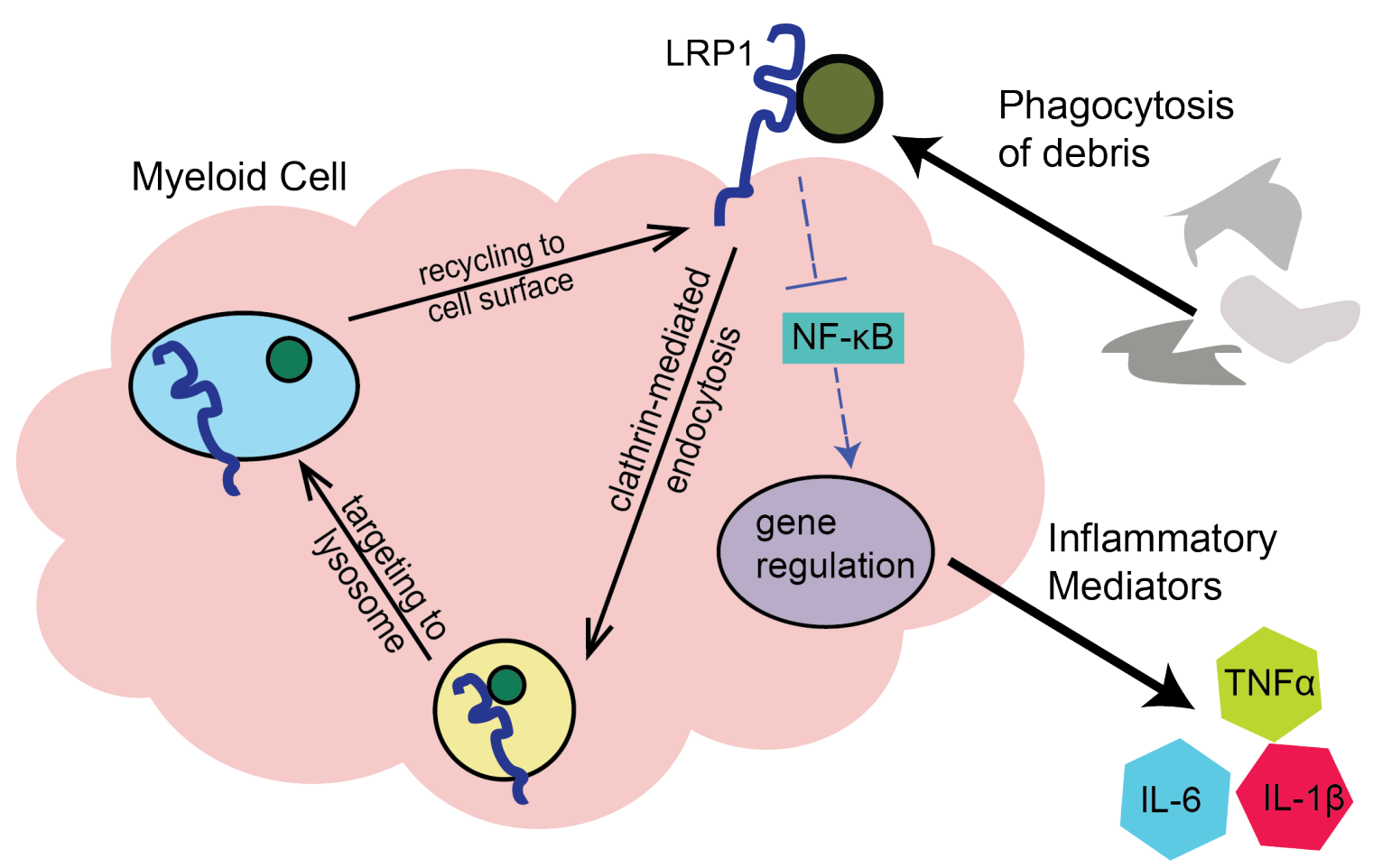

On myeloid cells, LRP1 is found on the cell surface, where it recognizes and binds to extracellular ligands and mediates phagocytosis of the ligands, including cell debris. LRP1 initiates clathrin-mediated endocytosis and targets its ligands to the lysosome, where acidification causes dissociation of the ligand from the receptor. The ligand is degraded and LRP1 is recycled back to the cell surface. LRP1 also involved regulating the production of inflammatory mediators, which it can do throughout its phagocytic cycle. 
Table 1: LRP1 functions as a scavenger receptor for multiple ligands

Adapted from Lillis et al., $2008^{120}$.

\begin{tabular}{|c|c|}
\hline Proteins involved in lipoprotein metabolism & \\
\hline $\begin{array}{l}\text { apolipoprotein E-enriched lipoproteins } \\
\text { (chylomicron and VLDL remnants) }\end{array}$ & \\
\hline$\beta$-VLDL & hepatic lipase \\
\hline lipoprotein lipase & sphingolipid activator protein \\
\hline Proteases and protease/inhibitor complexes & \\
\hline$\alpha 2 M^{*} \& \alpha 2 M^{*}$ protease complexes & \\
\hline pregnancy zone protein-protease complexes & \\
\hline aprotinin & pro-uPA, uPA \\
\hline uPA/PAI-1 complexes & tPA \\
\hline tPA/PAl-1 complexes & thrombin/PAI-1 complexes \\
\hline thrombin/anti-thrombin III & thrombin/heparin cofactor II \\
\hline thrombin/protease nexin-1 & neuroserpin \\
\hline neuroserpin/tPA complexes & elastase/a1-anti-trypsin \\
\hline C1s/C1q inhibitor & protease/protein C inhibitor \\
\hline MMP-9 & MMP-13 \\
\hline TSP-2/MMP-2 complexes & TFPI \\
\hline factor VIIa/TFPI & fVIII/fVIIIa \\
\hline factor Ixa & fXla/protease nexin- 1 \\
\hline$\beta$-amyloid precursor protein & \\
\hline Matrix proteins & \\
\hline thrombospondin-1 & thrombospondin-2 \\
\hline fibronectin & \\
\hline Intracellular proteins & \\
\hline RAP & HIV Tat protein \\
\hline Calreticulin & \\
\hline Growth factors & \\
\hline PDGF & midkine \\
\hline connective tissue growth factor (CTGF/CCN2) & \\
\hline transforming growth factor- $\beta$ & \\
\hline Others & \\
\hline circumsporozoite protein & gentamicin \\
\hline lactoferrin & polymycin B \\
\hline $\operatorname{ricin} \mathrm{A}$ & Pseudomonas exotoxin A \\
\hline saposin & complement $\mathrm{C} 3$ \\
\hline rhinovirus & collectins (via calreticulin) \\
\hline$\beta$ peptide (monomer) & Myoglobin \\
\hline
\end{tabular}




\section{Chapter 2: MATERIALS AND METHODS}

\section{Human Samples}

The human pathology study was approved by the Institutional Review Board of Mayo Clinic, Rochester, MN. Six clinically and pathologically confirmed multiple sclerosis autopsy cases were used in this study. Formalin-fixed paraffin-embedded $5 \mu \mathrm{m}$ thick sections were stained for H\&E, Luxol fast blue and periodic acid Schiff (LFB/PAS), and Bielschowsky's silver stain for routine pathology analysis. Immunohistochemistry was performed with the avidin-biotin-complex method according to the manufacturer's instructions (Vectorlab). The following primary antibodies are used: LRP1 (1:200, Abcam) myelin proteolipid protein (PLP, 1:500, Serotec), myelinassociated glycoprotein (MAG, 1:1000, Abcam), myelin oligodendrocyte glycoprotein (MOG, 1:1000, Abcam), CD68 (1:100, DAKO), and glial fibrillary acidic protein (GFAP, 1:100, DAKO). Steamed antigen retrieval with citrate buffer ( $\mathrm{pH}$ 6.0) was performed for LRP1, MAG, MOG, CD68, and GFAP. The demyelinating activity in the parenchyma for each block was evaluated in regions derived from the 6 cases, according to the myelin debris in macrophages as previously described ${ }^{155}$.

\section{Mice}

C57BL/6 mice with loxP sites flanking the LRP1 gene were crossed with $C x 3 c r 1^{\text {cre }}, C x 3 c r 1^{\text {creER }}$ and $L y s M^{\text {cre }}$ mice to generate $C x 3 c r 1^{\text {cre }}-L R P 1^{\mathrm{fl} / \mathrm{fl}}, C x 3 c r 1^{\mathrm{creER}}$. $L R P 1^{\mathrm{fl} / \mathrm{fl}}$ and $L y s M^{\mathrm{cre}}-L R P 1^{\mathrm{fl} / \mathrm{fl}}$ mice. To induce Cre recombinase activity, 4 to 6-weeks old $L R P 1^{\mathrm{fl} / \mathrm{fl}} C x 3 c r 1^{\mathrm{creER}}-L R P 1^{\mathrm{fl} / \mathrm{fl}}$ mice were injected twice i.p. with tamoxifen $(250 \mathrm{mg} / \mathrm{kg}$ in corn oil, Sigma) one week apart as described in the literature. For controls, littermates 
carrying loxP-flanked LRP1 alleles without Cre recombinase expression or age and sex matched mice with $C x 3 c r 1^{\text {creER }}$ activity without loxP-flanked LRP1 alleles, were used. All animal procedures were approved by the University of Virginia's Animal Care \& Use Committee.

\section{Mouse Genotyping}

The universal Cre genotyping was used to detect Cre from the generated mouse strains. The Cre transgene was maintained as a single copy in all experiments. Genotyping was performed on tail DNA which were lysed in $50 \mathrm{mM} \mathrm{NaOH}$ and neutralized with Tris-HCl. $1 \mu \mathrm{l}$ of $20 \mathrm{mM}$ primer dilution in a $20 \mu \mathrm{l}$ PCR reaction was used. The sequences are found in Table 2 .

\section{Cell Isolation from the CNS}

Brain and/or spinal cords were removed and digested in HBSS containing collagenase IV $(2 \mathrm{mg} / \mathrm{ml})$ and DNase $(20 \mathrm{U} / \mathrm{ml})$ for 45 minutes at $37^{\circ} \mathrm{C}$. Cells were isolated using a 30\%/70\% Percoll gradient (GE Healthcare). For adult primary microglia culture, cells were positively selected using CD11b magnetic microbeads (Miltenyi Biotec) after digestion.

\section{Experimental Autoimmune Encephalomyelitis (EAE)}

EAE was induced in female mice ( 8 to 12 weeks) by subcutaneous injection of $\mathrm{MOG}_{35-55}$ peptide $(100 \mu \mathrm{g}, \mathrm{CSBio})$ emulsified in complete Freund's adjuvant containing Mycobacterium tuberculosis (1mg/ml, BD). Pertussis toxin (200ng, List Biologicals) was administered i.p. on the day of and $1 \mathrm{~d}$ after MOG immunization. For experiments with $C x 3 c r 1^{\text {creER }}$ mice, EAE was induced 4 weeks after the tamoxifen treatment as previously described. For clinical evaluation, mice were scored daily: 0-no clinical disease, 1-limp 
tail, 2-hindlimb weakness, 3-hindlimb paralysis, 4-partial front limb paralysis, 5moribund. A schematic of EAE is presented in Figure 6.

\section{Antigen Recall Assay}

Antigen recall assay was performed as previously described. Briefly, single cell

suspensions were prepared from draining inguinal lymph nodes of $C x 3 c r 1^{\text {creER }} \operatorname{Lrp} 1^{\mathrm{fl} / \mathrm{fl}}$ and control mice 7 days post immunization with $\mathrm{MOG}_{35-55}$ peptide. Cells were treated with various concentrations of $\mathrm{MOG}_{35-55}$ peptide as indicated in the manuscript for $24 \mathrm{~h}$. ELISA was used to measure IFN- $\gamma$ and IL-17A production. To determine proliferation, $\operatorname{BrdU}(10 \mu \mathrm{M}, \mathrm{BD})$ was added to the culture media and BrdU incorporation was measured $16 \mathrm{~h}$ after treatment by flow cytometry.

\section{Blood Brain Barrier Assay}

6 hours prior to the assay, mice were injected i.p. with LPS $(6 \mathrm{mg} / \mathrm{kg})$. Heparin (20U/mouse) was administered and 30 minutes later, $2 \%$ sodium fluorescein $(200 \mu 1, \mathrm{Na}-$ F) injected i.p.. After another 30 minutes, the mice were perfused, the meninges removed, and the brains weighed and isolated in $50 \%$ trichloroacetic acid overnight at $4{ }^{\circ} \mathrm{C}$. The tissue was finely diced and homogenized. After centrifugation $\left(13,000 \times \mathrm{g}, 10 \mathrm{~min}, 4^{\circ} \mathrm{C}\right)$ and neutralization of the acid with $\mathrm{NaOH}(5 \mathrm{M}), 200 \mu \mathrm{l}$ of the clarified homogenate was plated in 96 well black/clear bottom plates and fluorescence measured. Data is expressed as amount of tracer per gram of tissue.

\section{Cuprizone}

8 week old mice were treated with $0.2 \%$ cuprizone [bis(cyclohexanone)oxaldihydrazone; 14690-25G, Sigma-Aldrich, Inc] mixed with chow 
for 5 weeks. Animals were then either immediately euthanized or removed from the diet and allowed to recover for 2 weeks.

\section{ELISA}

ELISA analyses for IL- 6 and TNF $\alpha$ were performed as previously described ${ }^{156}$. Antibodies used were: anti-mouse IL-6 $(0.5 \mu \mathrm{g} / \mathrm{mL}, \mathrm{MP} 5-20 \mathrm{~F} 3$, Biolegend); biotin antimouse IL-6 (1 $\mu \mathrm{g} / \mathrm{mL}$, MP5-32C11, Biolegend,); anti-mouse TNF $\alpha(0.5 \mu \mathrm{g} / \mathrm{mL}$, AF-410NA, R\&D systems); biotin anti-mouse TNF $\alpha(0.25 \mu \mathrm{g} / \mathrm{mL}$, BAF410 R\&D systems).

qPCR

RNA was extracted using the Isolate II Kit (Bioline) according to manufacturer's instructions and cDNA prepared with the iScript cDNA synthesis kit (Bio-Rad). qPCR was conducted with TaqMan primers (Applied Biosystems) for LRP1, IL-6, TNF- $\alpha$, Il$1 \beta$, and normalized to GAPDH. Reactions were run on a PikoReal PCR system (Thermo Fisher) with 2x NO-ROX Sensifast Mix or 2x SYBR Sensifast Mix (Bioline). Designed SYBR probes for the following murine genes are found in Table 3.

\section{Immunofluorescence}

Anesthetized mice were perfused transcardially with PBS, followed by $4 \%$ paraformaldehyde (PFA) prepared in PBS. Tissues were post-fixed for at least $24 \mathrm{~h}$ in $4 \%$ PFA, and transferred to $20 \%$ sucrose solution before sectioning. Cryosections $(40 \mu \mathrm{m})$ were permeabilized in $0.5 \%$ PBS-Tween for 15 minutes and washed twice with PBS. After blocking in 5\% serum in PBS for 2 hours at room temperature, sections were incubated overnight at $4^{\circ} \mathrm{C}$ with antibody against Iba-1 (ab5076, 1:400, Abcam), LRP1 (ab92544, 1:250, Abcam) and CD68 (14-0688, 1:50, eBioscience). For cuprizone treated mice, sections were stained with antibody against CCL1 (OP80, Millipore) and Olig2 
(MABN50, Millipore). After washing, sections were incubated with secondary antibodies conjugated to Alexa488 or Alexa647 (Life Technologies). Sections were mounted with Prolong Gold Anti-Fade Reagent containing DAPI (Life Technologies). All sections were imaged with a Leica TCS SP8 confocal microscope and analyzed with ImageJ. Sholl analysis was performed as described ${ }^{133}$.

\section{Immunohistochemistry}

Slides were deparaffinized using xylenes and an ethanol gradient. Heat induced antigen retrieval was performed for 10 min (Sodium Citrate Buffer, $\mathrm{pH}$ 6). Staining for CD68, Iba-1, and LRP1 was conducted using ImmPress reagents according to manufacturer's instructions (Vector lab). Adjacent sections were stained with LFB for demyelinating plaques.

\section{Preparation of Bone Marrow Derived Macrophages}

Mice were euthanized and dissected to retrieve femurs and tibiae. BMDM cells were cultured at $37^{\circ} \mathrm{C} 5 \% \mathrm{CO}_{2}$ in high glucose DMEM with L-glutamine (SH30022, GE Life Sciences) supplemented with 10\% fetal bovine serum (s12450, Atlanta Biologicals), sodium pyruvate (11360-070, Life Technologies), 1x Penicillin-Streptomycin (151490122, Life Technologies) and 30\% L929-conditioned media. Fresh media was supplemented at day 4 , and the cells were replated on day 6. In some experiments, BMDM were treated with LPS (Sigma) and other TLR ligands (InvivoGen) as described in the text.

\section{Immunoblotting}

Proteins were extracted in RIPA buffer supplemented with protease inhibitor cocktail (Roche) and the phosphatase inhibitor sodium orthovanadate (2mM). After 
incubation for 15 minutes on ice, the lysates were centrifuged $\left(13000 \times \mathrm{g}, 4^{\circ} \mathrm{C}\right)$ and protein quantified using BCA assay. Protein samples were run on a Protean TGX gel (Bio-Rad) and transferred to a PVDF membrane. After blocking with 5\% milk in TBSTween for $1 \mathrm{hr}$ at room temperature, membranes were incubated overnight with primary antibody. After washing, membranes were incubated with HRP conjugated secondary antibodies (Pierce) for $1 \mathrm{~h}$ at room temperature and developed with Western Lighting Plus ECL (Perkin Elmer).

\section{Flow Cytometry/Fluorescence Activated Cell Sorting}

For flow cytometry analysis, single cell suspensions were stained with antibody against CD45 (30-F11, eBioscience), CD11b (M1/70, eBioscience), TCR $\beta$, CD4, CD8, CD19, TNF $\alpha$ (MP6-XT22, eBioscience), Il-1ß (NJTEN3, eBioscience) and LRP1 (ab92544, 1:1000, Abcam). Flow cytometry analyses were performed on a 10-color Beckman Coulter Gallios flow cytometer and data were analyzed with FlowJo software

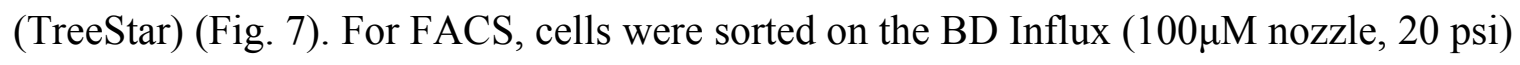
and purity verified to be $>98 \%$ for each cell population. FACS sorted myeloid cells were either frozen for RNA extraction or cultured in L929 media.

\section{Phagocytosis assays}

$\mathrm{pH}$ sensitive protein dyes were used to assess the phagocytic activity of myeloid cells. For preparation of fluorescent myelin, myelin was first purified from rat brain. Myelin was then labeled with CypHer5e NHS Ester (GE Healthcare Life Sciences) according to manufacturer's protocol. Lyophilized pHrodo-labeled ligands from bacteria (E.coli) and yeast (Zymosan) (Life Technologies) were resuspended according to 
protocol. Cells were plated at a density of $10 \mathrm{e}^{5} /$ well in a 96 well black walled plate with clear bottoms. The fluorescence over time was measured in a reader (SPECTRAmax).

\section{Cell Culture}

Unless otherwise specified, cells were cultured in DMEM media (high glucose, Invitrogen) supplemented with $10 \% \mathrm{FBS}$ and $1 \%$ Pen/Strep (Invitrogen) at $37^{\circ} \mathrm{C}, 5 \%$ $\mathrm{CO}_{2}$.

\section{Statistical analysis}

Statistical comparisons were conducted by unpaired Student's two-tailed t-test, Log-rank (Mantel-Cox) test, or two-way ANOVA using GraphPad Prism version 6.0. All results are expressed as means \pm sem. Results with a $p$-value $<0.05$ were considered significant. 


\section{Figures \& Tables}

Figure 6: Experimental protocol for experimental autoimmune encephalomyelitis (EAE)

a

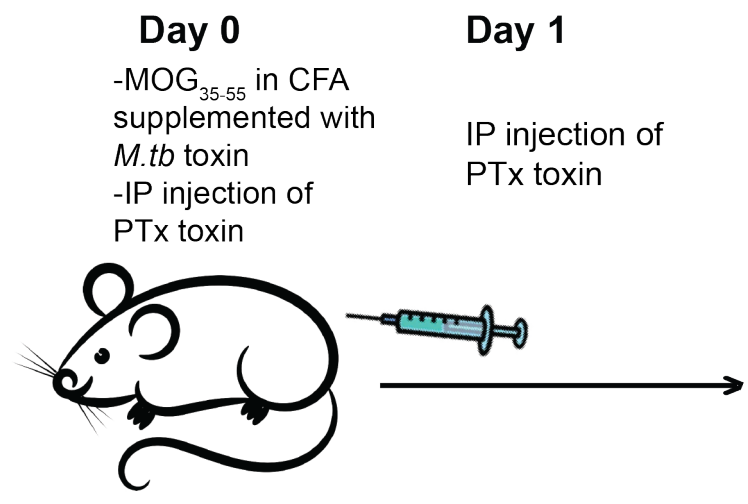

Day 7-Day 28

Monitor Clinical Signs of Paralysis

M.tb toxin

IP injection of -IP injection of

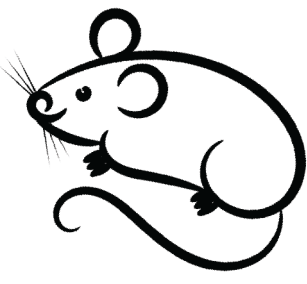

Clipart by AnimalsClipart.com

b

Score

Mouse Posture

0

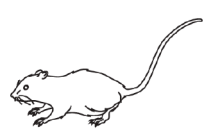

Normal

0.5

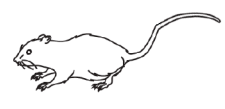

Weak tail

1

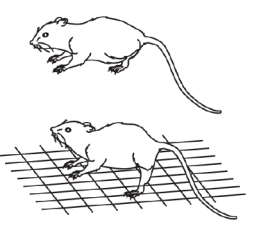

Paralyzed Tail

2

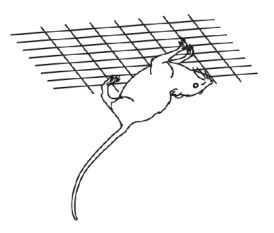

Mild Hindlimb

dysfunction

3

Severe Hindlimb

dysfunction

4

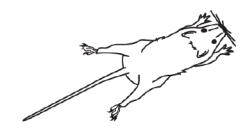

Hindlimb Paralysis

5

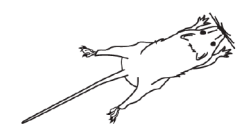

\section{Dead/Moribund}

a Schematic depicts immunization protocol for optimal EAE induction by active immunization in C57/BL6 mice. b Scoring criteria used during EAE (modified from Current Protocols in Neuroscience, 2001). 
Figure 7: Flow cytometry gating strategy to identify macrophages, microglia and

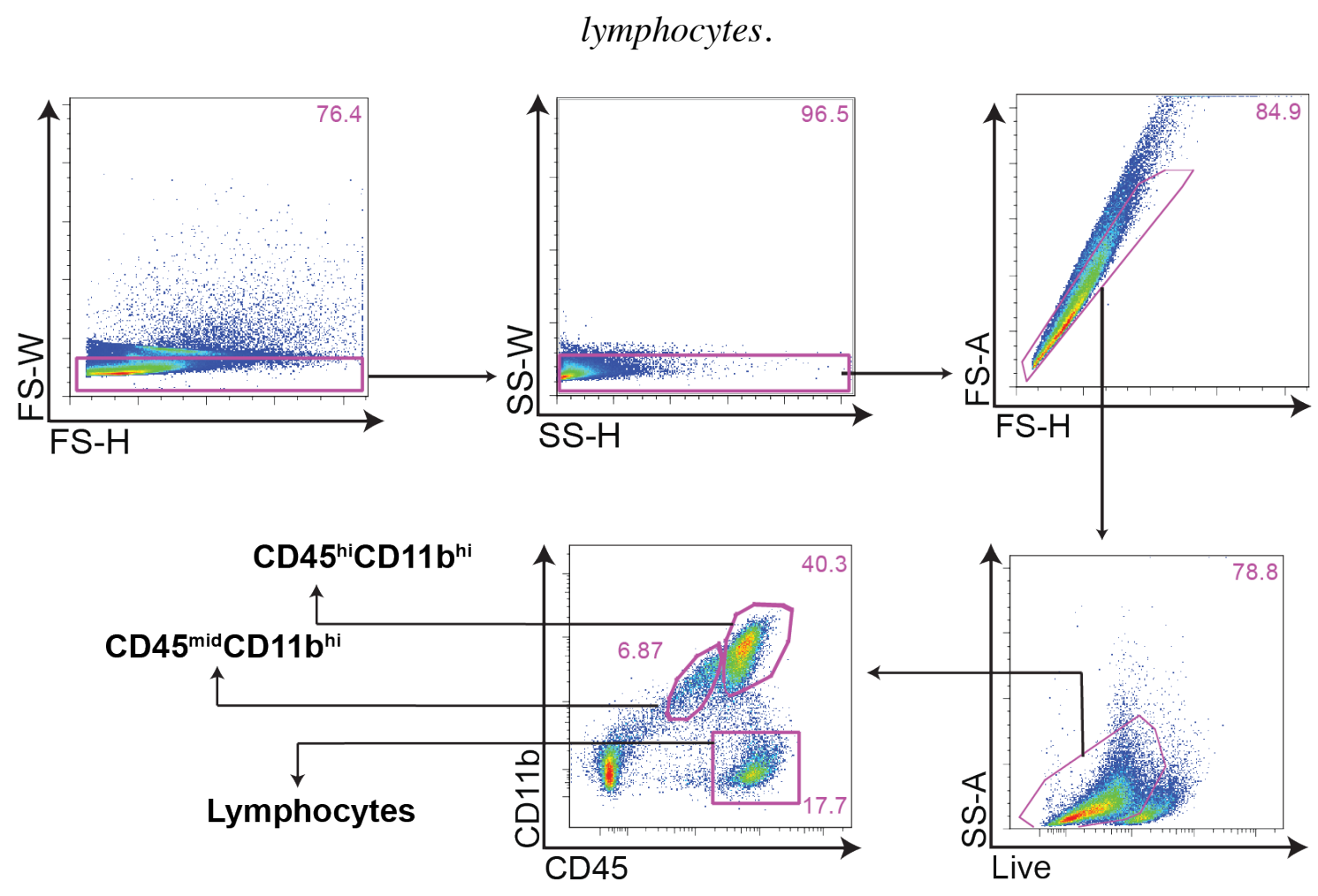

Singlet live cells are analyzed on a CD45 against CD11b plot and peripheral infiltrating myeloid cells $\left(\mathrm{CD} 45^{\mathrm{hi}} \mathrm{CD} 11 \mathrm{~b}^{\mathrm{hi}}\right)$, microglia $\left(\mathrm{CD} 45^{\mathrm{mid}} \mathrm{CD} 11 \mathrm{~b}^{\text {hi }}\right)$, and lymphocytes $\left(\mathrm{CD} 45^{\mathrm{hi}}\right.$ $\mathrm{CD} 11 \mathrm{~b}^{\text {neg }}$ ) identified. Lymphocytes were further classified as $\mathrm{T}$ and $\mathrm{B}$ cells by staining of TCR $\beta$ and CD19. 
Table 2. Sequencing primers

\begin{tabular}{|c|c|c|}
\hline Gene & Forward & Reverse \\
\hline LRP1 & CAT ACC CTC TTC AAA CCC CTT \\
CCT G & $\begin{array}{c}\text { GCA AGC TCT CCT GCT CAG } \\
\text { ACC TGG A }\end{array}$ \\
\hline CX3CR1 WT & TTCACGTTCGGTCTGGTGG & GGTTCCTAGTGGAGCTAGGG \\
\hline CX3CR1 Tg & CACGGGGGAGGCAGAGGGTTT & GCGGAGCACGGGCCACATTTC \\
\hline LysM Cre WT & TTACAGTCGGCCAGGCTGAC & CTTGGGCTGCCAGAATTTCTC \\
\hline LysM Cre Tg & CCCAGAAATGCCAGATTACG & CTTGGGCTGCCAGAATTTCTC \\
\hline $\begin{array}{c}\text { Cre } \\
\text { recombinase }\end{array}$ & $\begin{array}{c}\text { GCATTACCGGTCGATGCA } \\
\text { ACGAGTGATGAG }\end{array}$ & $\begin{array}{c}\text { GAGTGAACGAACCTGGTCGAA } \\
\text { ATCAGTGCG }\end{array}$ \\
\hline Internal Control & $\begin{array}{c}\text { TGGACAGGACTGGACCTCTGCTTTCC } \\
\text { TAGA }\end{array}$ & $\begin{array}{c}\text { TAGAGCTTTGCCACATCA } \\
\text { CAGGTCATTCAG }\end{array}$ \\
\hline
\end{tabular}

Table 3. List of SYBR qPCR probe sequences

\begin{tabular}{|c|c|c|}
\hline Gene & Forward & Reverse \\
\hline TFEB & TAACACGCTGCCCCTGTCTA & CTTCTGAGTCAGGTCGGCAG \\
\hline ATP6V1H & GCCAATGTTGAATCGCCAGG & CACATACTGAGAACTCTGTGAACTC \\
\hline CLCN7 & CGCCAGTCTCATTCTGCACT & GCTTCTCGTTGTGTGGAATCT \\
\hline CTSA & CCCTCTTTCCGGCAATACTCC & CGGGGCTGTTCTTTGGGTC \\
\hline HEXA & TCCGTCGTCACAGCTGAATG & CTCCAGACCTCGGAGAGCG \\
\hline LAMP1 & CAGCACTCTTTGAGGTGAAAAAC & ACGATCTGAGAACCATTCGCA \\
\hline HEXB & CTGGTGTCGCTAGTGTCGC & CAGGGCCATGATGTCTCTTG \\
\hline LAMP2 & ATGCCCCTCTGGGAAGTTCT & TGCACTGCAGTCTTGAGCTGT \\
\hline
\end{tabular}




\title{
Chapter 3: LRP1 EXPRESSION IN MICROGLIA IS PROTECTIVE DURING AUTOIMMUNITY
}

This chapter expands on the publication authored by Tzu-Ying Chuang, Yong Guo, Scott M. Seki, Abagail M. Rosen, David M. Johanson, James W. Mandell, Claudia F. Lucchinetti and Alban Gaultier. Acta Neuropathologica communications (2016)

\begin{abstract}
Multiple sclerosis (MS) is a devastating neurological disorder characterized by the autoimmune destruction of the central nervous system (CNS) myelin. While T cells are known orchestrators of the immune response leading to MS pathology, the precise contribution of CNS resident and peripheral infiltrating myeloid cells is less well described. Here I explore the myeloid cell function of LRP1, a scavenger receptor involved in myelin clearance and the inflammatory response, in the context of MS. Supporting its central role in MS pathology, I find that LRP1 expression is increased in the MS lesions in comparison to the surrounding healthy tissue. Using two genetic mouse models, I show that deletion of LRP1 in microglia, but not in peripheral macrophages, negatively impacts the progression of experimental autoimmune encephalomyelitis (EAE), an animal model of MS. I further show that this is increase in EAE disease severity is not due to haplodeficiency of the $\mathrm{Cx} 3 \mathrm{crl}$ locus. Furthermore, microglia lacking LRP1 adopt a pro-inflammatory phenotype characterized by amoeboid morphology and increased production of the inflammatory mediator TNF $\alpha$. I also show that LRP1 functions as a robust inhibitor of NF- $\mathrm{\kappa B}$ activation in myeloid cells via a
\end{abstract}


MyD88 dependent pathway, potentially explaining the increase in disease severity observed in mice lacking LRP1 expression in microglia. Taken together, our data suggest that the function of LRP1 in microglia is to keep these cells in an anti-inflammatory and neuroprotective status during inflammatory insult, including experimental autoimmune encephalomyelitis and potentially in MS.

\section{Introduction}

MS is an inflammatory autoimmune disease characterized by the destruction of myelin in the central nervous system (CNS) and irreversible neurodegeneration ${ }^{6}$. As discussed, there is great diversity in MS disease course and severity for individual patients. The etiology of MS is still unknown, and so a curative treatment still does not exist. However, the presence of demyelinating MS lesions in nearly $90 \%$ of patients shows evidence of dysregulated immune activity in the CNS. According to traditional models of disease pathogenesis, myelin destruction is initiated by myelin-reactive $\mathrm{T}$ cells infiltrating across the $\mathrm{BBB}$ into the $\mathrm{CNS}$, and is further amplified by the inflammatory response of myeloid cells, including the brain resident microglia and the brain infiltrating inflammatory macrophages ${ }^{37,157}$.

Although there are several lines of evidence indicating that microglia and macrophages participate in the pathogenesis of MS, their precise functions remain extremely controversial. On one hand, microglia are proposed to be protective and relatively immune silent in MS, when compared to macrophages ${ }^{158}$, while other studies demonstrate that microglia themselves can contribute to disease initiation. Indeed, mice with the microglia-specific deficiency in the activation of the transcriptional master 
regulator of inflammation, NF- $\kappa B$, are protected from EAE ${ }^{159}$. Mice exhibit exacerbated EAE disease when NF- $\mathrm{kB}$ transcriptional activity is constitutively active in infiltrating macrophages and exhibit an ameliorated disease course when NF- $\kappa \mathrm{B}$ activity is genetically ablated in infiltrating macrophages ${ }^{50,51}$. These results illustrate the importance of inflammatory signaling in myeloid cells during disease pathogenesis during EAE. Understanding the initiation, maintenance and resolution of inflammation in MS has been critical to development of currently available therapeutics, and continues to be a viable method for investigating novel mechanisms of regulating immune activity in the CNS. Strikingly, the presence of myelin and apoptotic debris in the MS lesion has also been shown to influence disease progression, as absence of debris clearance leads to lack of remyelination and subsequent neuronal death.

It has been previously reported that the presence of scavenger receptors in the MS lesion is greatly increased. Low-density lipoprotein receptor-related protein-1 (LRP1), or CD91, is a scavenger receptor involved in the removal of myelin debris, as well as necrotic and apoptotic cells ${ }^{131,133,160}$. There are increased levels of LRP1 mRNA expression at the rim of the MS lesion ${ }^{153}$. The increased expression of LRP1 may be linked to its phagocytic function, as the clearance of myelin debris generated during demyelination episodes is critical for the regenerative capacity of the CNS. Improper myelin debris clearance by microglia has been shown to delay recovery in a mouse model of demyelination ${ }^{95}$.

Besides mediating the removal of cellular debris, LRP1 can also directly influence cellular signaling pathways ${ }^{120}$. Our laboratory previously showed that LRP1 functions as a broad inhibitor of NF- $\mathrm{kB}$ activity and inflammatory mediator production in murine 
fibroblasts ${ }^{134}$. Because inhibition of NF- $\mathrm{KB}$ is known to offer protection during the experimental autoimmune encephalomyelitis (EAE), a mouse model of MS ${ }^{78-80,161}$, placement of LRP1 at the crossroads of inflammation and phagocytosis raises the possibility of its involvement as a central regulator of MS pathology.

In this work, I explore the precise contribution of LRP1 during MS, using EAE as an animal model. I hypothesized that LRP1's role as a scavenger receptor and regulator of inflammation could influence myeloid cell function. I show that LRP1 protein expression is increased in the myeloid and astrocytic compartments during active disease in MS, in comparison to the healthy CNS. To elucidate the role of LRP1 in myeloid cells, I induced EAE in mice lacking LRP1 in either the peripheral or resident myeloid cells by using the $L R P I^{\mathrm{fl} / \mathrm{fl}} L y s M^{\text {cre }}$ or tamoxifen inducible $L R P I^{\mathrm{fl} / \mathrm{ll}} C x 3 c r I^{\text {creER }}$ mice. To our surprise, deletion of LRP1 in peripheral macrophages had no effect on the disease severity, whereas disease severity was intensified in mice lacking LRP1 specifically in microglia, suggesting a protective role for LRP1 expression. Further experiments revealed that, at baseline, microglia deficient in LRP1 adopt a pro-inflammatory phenotype and exhibit morphological changes that parallel those observed in wild-type microglia under inflammatory conditions. On a mechanistic level, our results suggest that the increased pathology in mice with LRP1 deficient microglia originates from an uncontrolled NF-KB activity through specifically regulating the MyD88 pathway. I also show that LRP1 deficient myeloid cells may have deficits in myelin phagocytosis. Taken together, our results demonstrate that LRP1 has a protective role in microglia and that the alteration of their function can drastically impact EAE outcome. As I found that LRP1 expression is increased on myeloid cells during MS, developing ways to promote the 
activity of LRP1 - either by directly targeting the receptor or one of its downstream adaptor protein pathways, could potentially be applied to therapeutics for MS.

\section{Results}

LRP1 expression is increased in MS lesions

Our previous studies demonstrated that LRP1 functions as a receptor for myelin phagocytosis and a broad inhibitor of inflammation, two key functions linked with MS pathogenesis ${ }^{131,134}$. Because our lab previously observed increased expression of LRP1 in the CNS during an animal model of MS, EAE ${ }^{131}$, I decided to probe the potential function of LRP1 during the human disease. I began by examining LRP1 expression in MS lesions at different stages of demyelinating activity in collaboration with the Lucchinetti lab at Mayo Clinic who conducted the clinical and pathological analysis. The results from a total of 6 MS autopsies examined are summarized in Table 4.

By immunohistochemistry, we stained for myelin proteolipid protein (PLP), CD68, GFAP, and LRP1. In the normal appearing periplaque gray matter, LRP1 was mainly detected in neurons (Fig. 8a, lower row), in agreement with a previous publication 162. However, within the early active lesions, characterized by the presence of CD68+ myeloid cells containing myelin debris and GFAP+ reactive astrocytes, LRP1 expression was increased (Fig. 8a, upper row). Morphological analysis suggested that LRP1 expression was most prominent in myeloid cells (arrow) and astrocytes (arrowhead) in the early active lesion. To confirm that myeloid cells express LRP1 within the active lesion, I performed double immunofluorescence with antibodies against LRP1 and CD68. Within the lesion, CD68+ cells are also LRP1+, while LRP1 is not detectable within the 
myeloid cells outside the lesion, confirming our immunohistochemistry results (Fig. 8b). LRP1 immunoreactivity in myeloid cells was also not present in inactive lesions (Table 4). Taken together, my results demonstrate that LRP1 expression in myeloid cells and astrocytes is increased during MS pathology.

\section{Generation of Microglial LRP1 deficient mice}

In order to generate mice that have myeloid populations deficient in LRP1, I crossed our $\operatorname{Lrp} 1^{\mathrm{fl} / \mathrm{fl}}$ strain to the $C x 3 c r 1^{\text {cre }}$ and $C x 3 c r 1^{\text {creER }}$ strains kindly shared with us by Dr. Steffen Jung. I injected the $C x 3 c r 1^{\text {creER }} \operatorname{Lrp}^{\mathrm{fl} / \mathrm{fl}}$ strain with tamoxifen and after 4 weeks, analyzed their CNS cell composition by flow cytometry. I validated that at baseline, the novel $C x 3 c r 1^{\text {creER }}-\operatorname{Lrp} 1^{\mathrm{fl} / \mathrm{fl}}$ strain did not have differences in CNS microglial cell composition by flow cytometry (Fig. 9a). There was no difference in the number or percentage of microglia, defined as microglia $\left(\mathrm{CD} 45^{\mathrm{mid}} \mathrm{CD} 11 \mathrm{~b}^{\text {hi }}\right.$ cells), in our $C x 3 c r 1^{\text {creER }}$ $\operatorname{Lrp}^{\mathrm{fl} / \mathrm{fl}}$ strain (Fig. 9b). Staining the cells for LRP1 expression revealed clear deletion of LRP1 in the microglia of the brain and the spinal cord. (Fig 9c, left 2 panels). I also made bone marrow-derived macrophages from these same mice and analyzed their LRP1 expression one week later at harvesting. Flow cytometry reveals that LRP1 expression is intact in the $C x 3 c r 1^{\text {creer }}-\operatorname{Lrp} 1^{\mathrm{fl} / \mathrm{fl}}$ macrophages (Fig 9c, right panel). I also used flow cytometry to compare expression between $C x 3 c r 1^{\text {creER }}-\operatorname{Lrp} 1^{\mathrm{fl} / \mathrm{fl}}, C x 3 c r 1^{\mathrm{cre}}-\operatorname{Lrp} 1^{\mathrm{fl} / \mathrm{fl}}$, and $\operatorname{LrpI}^{\mathrm{fl} / \mathrm{fl}}$ control microglia (Fig. 9d). Both constitutive and inducible forms of $\mathrm{Cx} 3 \mathrm{cr} I^{\text {cre }}$ efficiently excise LRP1 in microglia, as expression of LRP1 is decreased to nearly that of the fluorescence minus one (FMO) control.

Next, I confirmed LRP1 deletion in $C x 3 c r 1^{\text {cre }}-\operatorname{Lrpl}^{\mathrm{fl} / \mathrm{fl}}$ macrophages by qPCR (Fig. 9e) and western blot (Fig. 9f). As expected, $C x 3 c r 1^{\text {cre }}-L r p 1^{\text {fl/fl }}$ macrophages have 
sustained deletion of LRP1. I also concurrently analyzed LRP1 expression in $C x 3 c r 1^{\text {creER }}$ $\operatorname{Lrp} 1^{\mathrm{fl} / \mathrm{fl}}$ macrophages by western blot, confirming my flow cytometry results that these macrophages have similar expression of LRP1 as control. As I also use the Lys $M^{\text {cre }}$ $\operatorname{Lrp} 1^{\mathrm{fl} / \mathrm{fl}}$ mouse, which has LRP1 deleted in its peripheral myeloid cells, I immunoblotted bone marrow macrophages from these mice side by side with macrophages from $C \times 3 c r 1^{\text {cre }}-\operatorname{Lrp} 1^{\mathrm{fl} / \mathrm{fl}}$ mice (Fig. 9g). Although both strains of cre efficiently decrease the amount of LRP1 expression by western blot, $C x 3 c r 1^{\text {cre }}-\operatorname{Lrpl}^{\mathrm{fl} / \mathrm{fl}}$ macrophages have a lower amount of LRP1 expression remaining when compared to $L y s M^{\text {cre }}-L r p 1^{\mathrm{fl} / \mathrm{fl}}$ macrophages, suggesting that it mediates more efficient excision in macrophages.

Microglial, but not peripheral macrophage LRP1, is protective during experimental autoimmune encephalomyelitis

Myeloid cells involved in MS pathology are from two different origins, the yolk sac derived CNS resident microglia ${ }^{53}$ and the peripheral infiltrating macrophages. To explore the function of myeloid LRP1, I used the well-accepted animal model of MS, EAE, in two different mice strains lacking LRP1 either in peripheral myeloid cells or in microglia. To study the function of LRP1 in peripheral myeloid cells, I induced EAE in LRP1 deleted $L y s M^{\mathrm{cre}}-\operatorname{Lrp} 1^{\mathrm{fl} / \mathrm{fl}}$ or the control $\operatorname{Lrp} 1^{\mathrm{fl} / \mathrm{fl}}$ mice with the myelin oligodendrocyte glycoprotein $\left(\mathrm{MOG}_{35-55}\right)$ peptide and scored the mice daily. While LysM is highly expressed in peripheral myeloid inflammatory cells, LysM expression in microglia is variable ${ }^{32,159}$. Using $L y s M^{\text {cre }}-L r p 1^{\mathrm{fl} / \mathrm{fl}}$ mice ${ }^{135}$, I observed complete deletion of LRP1 in bone marrow derived macrophages (BMDM), while I observed little to no decrease in basal microglial LRP1 expression, as demonstrated by flow cytometry (Fig. 10a). Given that our lab previously showed that macrophage LRP1 functions as an inhibitor of 
inflammation, I was surprised to find that deletion of LRP1 in peripheral myeloid cells in $L y s M^{\mathrm{cre}}-L r p I^{\mathrm{f} / / \mathrm{fl}}$ mice had no effect on the clinical score or incidence of EAE (Fig. 10b-c).

Next, I generated a new mouse strain to study the role of LRP1 in microglia during EAE. The $L r p 1^{\mathrm{fl} / \mathrm{fl}}$ strain ${ }^{163}$ was crossed to the recently developed $\mathrm{Cx} 3 \mathrm{cr} 1^{\mathrm{creER}}$ strain ${ }^{55}$ to generate $C x 3 c r 1^{\text {creER }}-L r p I^{\mathrm{fl} / f l}$ animals. Because CX3CR1 is expressed in microglia and monocytes ${ }^{159}$, tamoxifen treatment initially leads to the deletion of LRP1 in both cell types. However, the pool of circulating monocytes that can give rise to macrophages during inflammation is continuously renewed by progenitors from the bone marrow, while the long-lived brain resident microglia are not replenished and remain LRP1 deleted ${ }^{37,159}$. Indeed, 1 month after the administration of tamoxifen, I could still detect deletion of LRP1 in microglia, while LRP1 expression in bone marrow derived macrophages was comparable to control cells (Fig. 10d). EAE was induced by immunization with $\mathrm{MOG}_{35-55}$ peptide. LRP1 deletion in microglia results in a significant increase in clinical scores and overall incidence of EAE (Fig. 10e-f). My results suggested that LRP1 expression in microglia has a protective role in EAE, significantly reducing the disease severity.

To confirm that $L y s M^{\mathrm{cre}}-\operatorname{Lrp} I^{\mathrm{fl} / \mathrm{fl}}$ mice did not have worse EAE disease compared to control with increased immune activation, I increased the amount of mycobacterium to induce stronger EAE disease course. I found that the disease course was similar, confirming our findings that LRP1 deletion in peripheral myeloid cells does not affect EAE progression (Fig. 11a). I also addressed questions concerning literature indicating that CX3CR1 haploinsufficiency may cause exacerbation of EAE by inducing disease in $C x 3 c r 1^{\text {creER }}-\operatorname{Lrp} 1^{\mathrm{fl} / \mathrm{fl}}$ and $\operatorname{Lrp} 1^{\mathrm{fl} / \mathrm{fl}}$ animals that had not been injected with tamoxifen. The 
induction of EAE in these animals showed no significant difference between groups, indicating that $\mathrm{CX} 3 \mathrm{CR} 1$ haplodeficiency does not contribute to EAE progression (Fig. 11b).

Demyelination and peripheral immune cell recruitment are elevated in mice lacking $L R P 1$

To explore the pathological conditions leading to an exacerbated disease course in EAE mice lacking LRP1 expression in microglia, I performed histological analysis of the spinal cord during the chronic phase of the disease (day 30). Myelin staining of the spinal cord sections with Luxol Fast Blue revealed that $C x 3 c r 1^{\text {creER }}-\operatorname{Lrp} I^{\mathrm{fl} / \mathrm{fl}}$ mice have significantly more white matter demyelination than the control group (Fig. 12a). This difference was statistically significant at all of the spinal cord levels that were examined: Lumbar, thoracic and cervical levels. Immune cells are responsible for driving demyelination during EAE. To explore if LRP1 deficiency in microglia could alter immune cell numbers, I performed flow cytometry and immunohistochemical analysis of the spinal cords at the onset of disease (day 15). Histologically, I find increased numbers of $\mathrm{CD} 3+\mathrm{T}$ cells and Iba1+ cells in the $C x 3 c r 1^{\text {creER }}-L r p 1^{\mathrm{fl} / \mathrm{fl}}$ mice at disease onset (Fig. $12 b-c)$, especially evident in the dense infiltrates in the white matter of the spinal cord. By flow cytometry, my results reveal that the number of $\mathrm{T}$ cells and peripheral myeloid $\left(\mathrm{CD} 11 \mathrm{~b}^{\mathrm{hi}} \mathrm{CD} 45^{\mathrm{hi}}\right.$ ) cells was significantly increased in animals lacking LRP1 (Fig. 12d-e). There was a trend towards increased numbers of microglia $\left(\mathrm{CD} 45^{\mathrm{mid}} \mathrm{CD} 11 \mathrm{~b}^{\mathrm{hi}}\right)$ and $\mathrm{B}$ cells $\left(\mathrm{CD} 19^{+}\right)$(Fig. 12f-g). Of note, the expression levels of activation marker MHC II was the same between the groups on both microglia and macrophages, suggesting that the 
activation status of the cells was not measurably different by MHC II expression (Fig. 12h-i). Taken together, these results show that mice lacking LRP1 in microglia had increased EAE susceptibility that is associated with robust demyelination and increased infiltration of immune cells from the innate and adaptive arms of the immune system.

Lymphocyte activation is not affected in the absence of LRPI

LRP1 is a multifunctional receptor with roles that could impact EAE progression at different levels ${ }^{120}$. As LRP1 functions in antigen presentation ${ }^{164}$, and can be expressed in subsets of antigen presenting cells (APC) in the periphery ${ }^{33}$, I investigated the antigen recall response of inguinal lymph node $\mathrm{T}$ cells in the $C x 3 c r 1^{\text {creER }}-\operatorname{Lrp} 1^{\mathrm{fl} / \mathrm{fl}}$ mice 7 days after immunization. Treatment with $\mathrm{MOG}_{35-55}$ peptide did not reveal any significant differences in T cell production of IFN- $\gamma$ and IL-17A, as determined by ELISA (Fig. 13ab). Furthermore, $\mathrm{T}$ cell proliferation measured by $\mathrm{BrdU}$ incorporation was also comparable to control cells (Fig. 13c). Taken together, these data suggest that increased disease severity in EAE for $C x 3 c r 1^{\text {creER }}-\operatorname{Lrp} 1^{\mathrm{f} / / \mathrm{fl}}$ mice is not linked to impaired antigen presentation in the periphery.

Function of the blood brain barrier is intact in mice lacking microglial LRP1

Blood brain barrier (BBB) permeability disruption is an essential event in providing access to peripheral lymphocyte infiltration into the CNS during EAE ${ }^{165}$, and microglia are known to modulate the function of the BBB ${ }^{166,167}$. To investigate if LRP1 deletion in microglia can impact BBB permeability, I induced microgliosis in $C x 3 c r 1^{\text {creER }}-\operatorname{Lrp} 1^{\mathrm{fl} / \mathrm{fl}}$ and control mice by injecting LPS, as previously described ${ }^{167}$. LPS 
administration leads to increased extravasation of fluorescein in comparison to the basal level, as expected ${ }^{166}$. However, BBB permeability was not affected by the status of LRP1 expression in microglia (Fig. 13d). These results suggest that LRP1 function in microglia is likely not critical for the maintenance of the BBB function.

Microglial morphology is altered by LRPI deficiency

Since our results suggested that $\mathrm{T}$ cell and BBB function is normal in the absence of LRP1 expression in CX3CR1+ cells, I hypothesized that the increased disease severity is due to an intrinsic function of LRP1 in microglia. Therefore, I analyzed the morphology of microglia in the cortex of $C x 3 c r 1^{\text {creER }}-\operatorname{Lrp} 1^{\mathrm{fl} / \mathrm{fl}}$ and control mice at the resting state, and after inducing microgliosis via peripheral administration of low dose LPS for 4 consecutive days, as described ${ }^{168}$. I chose this model instead of EAE because of the lack of good markers to differentiate microglia from peripheral myeloid cells by immunochemistry. Because this model of neuroinflammation lacks recruitment of peripheral immune cells, a more focused analysis of microglia morphology is possible ${ }^{168}$. Brain sections were stained with Ibal specific antibody and microglial ramifications were quantified by Sholl analysis in the cortex (Fig. 14a-b). In the healthy control mice, microglia appear thin and ramified (Fig. 14b, grey curve). Surprisingly, microglia lacking LRP1 expression appeared more amoeboid than the control cells at baseline, with a denser cell body and thickened proximal dendrites (Fig. 14b, grey vs. orange curves). Whereas LPS administration in control mice leads to the transition of microglial morphology into the bushy and thickened appearance (Fig. 14b, grey vs black curves), LPS had no effect on the appearance and branching of LRP1 deficient microglia (Fig. 
$14 \mathrm{~b}$, orange vs red curves). LPS administration significantly increased the soma size of control microglia, but not the microglia lacking LRP1 expression (Fig. 14c). Quantification of the area covered by Iba1+ cells confirms that LRP1 deletion leads to microglial hypertrophy. The morphology of these LRP1 deficient microglia are similar in appearance to the microglia observed in LPS treated control mice (Fig. 14d). These morphological changes were not associated with an overall change in numbers of microglia (Fig. 14e). Furthermore, LRP1 status in microglia did not affect the weight of the animals injected with LPS, suggesting that systemic inflammatory cues were similar between the cohorts, unlike the altered morphology found in the CNS (Fig. 15). Our results suggest that the removal of LRP1 alters microglial morphology in a manner similar to treatment with LPS, a potent neuroinflammatory stimulus.

\section{Microglia lacking LRP1 have a proinflammatory signature}

One potential mechanism by which microglia lacking LRP1 could affect EAE pathology is through increased production of inflammatory mediators, as LRP1 functions as an inhibitor of the inflammatory response ${ }^{134}$. To test the inflammatory response in LRP1 deficient microglia, I isolated primary microglia from $\operatorname{Lrp} 1^{\mathrm{fl} / \mathrm{fl}}$ and $C x 3 c r 1^{\mathrm{cre}}-$ $\operatorname{Lrp} 1^{\mathrm{fl} / \mathrm{fl}}$ mice and analyzed their production of $\mathrm{TNF} \alpha$, a key cytokine involved in EAE pathology ${ }^{156}$. LPS stimulation of LRP1 deficient microglia results in increased production of TNF $\alpha$ at both the transcript (Fig. 16a) and the protein level (Fig. 16b). This increase in TNF $\alpha$ is comparable to the one observed in primary cultures of BMDM (Fig. $16 \mathrm{c}-\mathrm{d}$ ), as our lab has previously reported ${ }^{134}$. In BMDM, the pro-inflammatory signature generated by the lack of LRP1 also extends to IL-1 $\beta$ and IL-6 production (Fig. 16e-f). 
More importantly, this pro-inflammatory signature was also observed in a CNS homogenate from $C x 3 c r 1^{\text {creER }}-L r p 1^{\mathrm{fl} / f l}$ mice at the disease onset, as detected by $\mathrm{qPCR}$ (Fig. 16g). To further confirm that this pro-inflammatory signature is reflected in the microglia population of $C x 3 c r 1^{\mathrm{creER}}-\operatorname{Lrp} 1^{\mathrm{fl} / \mathrm{fl}}$ mice, I sorted the microglia from the CNS of mice at baseline and during EAE disease onset. qPCR analysis reveals that $C x 3 c r 1^{\text {creER }}$ $\operatorname{Lrp} 1^{\mathrm{fl} / \mathrm{fl}}$ microglia have a trend towards increased expression of inflammatory cytokines IL-6 and TNF $\alpha$, as well as proteins involved in the production of reactive oxygen species, iNOS and Arginine (Fig. 17). Taken together, these results demonstrate that, in the absence of LRP1, microglia adopt a pro-inflammatory phenotype that could contribute to increased pathology in EAE.

\section{LRP1 blocks NF- $\kappa B$ function in myeloid cells}

Production of cytokines, like TNF $\alpha$, IL-6 and IL-1 $\beta$, is tightly controlled via signaling through NF- $\kappa \mathrm{B}$, a transcriptional master regulator of the inflammatory response ${ }^{169}$. Indeed, recent studies have demonstrated that blocking NF- $\mathrm{B}$ activation specifically in microglia is sufficient to offer protection during $\mathrm{EAE}^{159}$. Previous reports have highlighted the fact that LRP1 can act as an inhibitor of inflammation through regulation of NF- $\kappa B$ activity ${ }^{134}$. I therefore hypothesized that LRP1 may function as a regulator of $\mathrm{NF}-\kappa \mathrm{B}$ mediated inflammatory response in microglia. Because the inflammatory mediator production appears similar in cultured microglia and BMDM lacking LRP1 (Fig. 16), I began by treating $\operatorname{Lrp} 1^{\mathrm{fl} / \mathrm{fl}}$ and $C x 3 c r 1^{\mathrm{cre}}-\operatorname{Lrp} 1^{\mathrm{fl} / \mathrm{fl}}$ BMDM with LPS and collecting protein extracts at several time points after stimulation. Protein extracts were analyzed for the phosphorylation status of the NF- $\kappa \mathrm{B}$ subunit p65. As is shown in Fig. 
18a, LPS stimulated LRP1 deficient macrophages have increased levels of phosphorylated p65 (p-p65), in comparison to control cells. LPS is the only Toll-like receptor (TLR) ligand known to activate two different signaling pathways: the MyD88 and the TRIF pathway ${ }^{169}$. To elucidate which arm of the signaling cascade is regulated by LRP1, I treated BMDM with a panel of TLR ligands that are associated with the TRIF (TLR3 ligand: Poly I:C), or the MyD88 adaptor response (TLR1 and 2 ligand: Pam3CSK4; TLR5 ligand: FLA-ST; TLR6 ligand: FSL-1; TLR9 ligand: ODN1826) ${ }^{170}$. BMDM were treated for $3 \mathrm{~h}$ and IL- 6 expression was determined by qPCR analysis. IL- 6 expression was increased in LRP1 deficient macrophages when the MyD88 pathway activated by TLR 1, 2, 5, 6, and 9. On the contrary, TLR3-mediated production of IL-6 was not affected by the status of LRP1, showing that LRP1 may inhibit the NF-kB signaling pathway in a MyD88, but not TRIF dependent manner (Fig. 18b). Together, these results suggest that LRP1 functions as a regulator of NF- $\mathrm{kB}$ activity through modulation of the MyD88 dependent arm of the inflammatory response. This function of LRP1 might explain why its removal in the microglial compartment is detrimental during CNS autoimmunity. 


\section{Discussion:}

In this study, I explore the function of LRP1 expression in myeloid cells during multiple sclerosis (MS) using the mouse model EAE. To begin, I have made the novel observation that LRP1 protein expression is significantly increased in human MS lesions, compared to normal appearing brain tissue. This finding corroborates with the previous work of Hendrickx et al. on the increased mRNA expression of scavenger receptors, including LRP1, in the MS lesion ${ }^{153}$. I find that the cellular compartments involved in the increase of LRP1 immunoreactivity included the myeloid cells and astrocytes. This observation is also in agreement with our group's previous work showing that LRP1 expression is upregulated during EAE in the spinal cord ${ }^{131}$. LRP1 expression increase by glia is not limited to MS, as previous work has demonstrated a similar pattern during CNS injury and neoplasia ${ }^{171}$. All of these disease conditions involve an increased proinflammatory environment in the CNS, with numerous inflammatory inputs. This suggests that LRP1 expression in glial cells is altered during CNS inflammation, and also during MS. In this study, we examined the functional role of LRP1 in the myeloid cells in the CNS during a mouse model of MS. In addition to myeloid cells, however, astrocytes also have increased expression of LRP1 in the MS lesion. Astrocytes are the most abundant glial cell type, and reactive astrocytes are also a feature of CNS inflammation. In addition to producing IL-6, IL-1 $\beta$, and TNF $\alpha$, astrocytes can also control the integrity of the blood brain barrier by acting on endothelial cells and tight junctions ${ }^{172}$. Therefore, although our study centered on the LRP1 function in myeloid cells, future studies will be needed to understand the role of LRP1 in astrocytes in neuroinflammation. 
Here, we have explored the contribution of LRP1 expression during EAE in two distinct myeloid cell populations: peripheral macrophages and microglia. The $L y s M^{\text {cre }}$ promoter allowed us to delete LRP1 expression in myeloid cells from the periphery, including monocyte-derived macrophages and neutrophils. To our surprise, we have discovered that LRP1 excision in peripheral macrophages had no detectable impact on EAE progression. This result contrasted with the consequence of LRP1 deletion in microglia, which leads to a significant worsening of disease progression in EAE.

We, and others, have previously demonstrated that macrophages lacking LRP1 expression display a pro-inflammatory phenotype in vitro, characterized by increased production of inflammatory mediators, chemokines and a M1-type macrophage skewing 134, 138, 143. This molecular mechanism suggested that the removal of LRP1 in macrophages could cause unbridled inflammatory activity, resulting in a more severe EAE disease course. Therefore, we were surprised to find that LRP1 deletion in Lys $M^{\text {cre }}$ $\operatorname{Lrp} \mathrm{f}^{\mathrm{f} / \mathrm{fl}}$ mice did not impact disease severity in two distinct EAE protocols, varying in the amount of mycobacterium used for the preparation of the complete Freund's adjuvant emulsion. One potential explanation for these observations is that the peripheral immune cells are primed for a powerful inflammatory response prior to their entry into the CNS, and the removal of LRP1 does not alter their function in the diseased tissue. Future studies are necessary to distinguish whether LRP1 deletion in peripheral myeloid cells alters their function under homeostatic conditions.

On the contrary, ablation of LRP1 in microglia had a significant impact on EAE disease severity and progression. Microglia are the resident myeloid cells of the CNS and their function in EAE remains actively debated; while some studies suggest that they 
actively participate in disease initiation and peripheral immune cell recruitment ${ }^{159,173}$, others show that microglia remain relatively inert during EAE ${ }^{158}$. Microglia have also been reported to express higher levels of the LRP1 transcript, compared to the peripheral macrophages, in both EAE and the homeostatic conditions ${ }^{65,147}$. This suggests that although both microglia and peripherally derived macrophages are professional phagocytes present in the CNS during EAE, they may have distinct biological roles related to the relative abundance of LRP1 located on their cell surface.

We proposed that microglia lacking LRP1 acquire an inflammatory phenotype that leads to disease exacerbation in EAE. In agreement with this hypothesis are our findings that LRP1 deficient microglia appear amoeboid and hypertrophic even in the absence of ongoing disease. These microglia have the distinct hallmarks of inflammation as indicated by increased soma size and thickening of the cellular processes. Multiple groups have described this transition from a ramified morphology to a dense, compact appearance by microglia as a clear sign of inflammation ${ }^{168,174}$. The fact that LRP1 deficient microglia appear activated in the healthy brain suggests that they are primed to produce a more robust inflammatory response. In response to in vivo stimulation by LPS, the LRP1 deficient microglia do not become appreciably more ramified as seen by morphological analysis. When isolated from the CNS via enzymatic digestion and treated with LPS in vitro, microglia lacking LRP1 secrete more TNF $\alpha$, a key inflammatory cytokine involved in EAE pathology ${ }^{156}$. These findings suggest that LRP1 deficient microglia are more proinflammatory at baseline.

On a mechanistic level, we propose that LRP1 is an inhibitor of NF-KB signaling in myeloid cells, building on previous studies from our laboratory regarding the role of 
LRP1 in primary mouse fibroblasts and fibroblast cell lines ${ }^{134,138}$. Here for the first time, we show clear evidence that LRP1 regulates the phosphorylation, and thus activation, of the canonical NF- $\kappa \mathrm{B}$ signaling pathway. A time course reveals that this activation can be observed within 15 minutes of LPS treatment, indicating a rapid cellular signaling response towards LPS.

Since LRP1 resides on the cell surface and is known to interact with a myriad of signaling receptors, we decided to investigate whether LRP1 differentially regulates members of the TLR family. TLR4, and by extension its ligand LPS, is the only member of this family to use both MyD88 and TRIF dependent signaling pathways. Our results indicate that LRP1 only influences the MyD88 arm of TLR signaling, suggesting that LRP1 only has an effect on the extracellular TLR ligands ${ }^{169}$. Further studies are needed to understand if the role of LRP1 as an NF- $\kappa \mathrm{B}$ inhibitor intersects with LRP1 function in the removal of myelin debris.

\section{Acknowledgement of co-author contributions}

TYC designed, performed and analyzed the research and wrote the paper. YG, SS, $\mathrm{AR}, \mathrm{DJ}, \mathrm{JM}$ and $\mathrm{CF}$ performed research. AG designed and analyzed the research and wrote the paper. All authors read and approved the manuscript. 
Table 4: LRP1 expression in MS

\begin{tabular}{llll}
\hline \multirow{2}{*}{$\begin{array}{l}\text { Lesion stage from MS } \\
\text { autopsy }\end{array}$} & \multicolumn{3}{l}{ LRP1 expression in the Lesion } \\
\cline { 2 - 4 } & Myeloid Cells & Neurons & Astrocytes \\
\hline Early Active & $100 \%(2 / 2)$ & $\mathrm{NA}^{\mathrm{a}}(0 / 2)$ & $100 \%(2 / 2)$ \\
Late Active & $0 \%(0 / 1)$ & $\mathrm{NA}^{\mathrm{a}}(0 / 1)$ & $100 \%(1 / 1)$ \\
Periplaque White Matter & $0 \%(0 / 5)$ & $\mathrm{NA}^{\mathrm{a}}(0 / 5)$ & $100 \%(5 / 5)$ \\
Non-Affected White Matter & $0 \%(0 / 5)$ & $\mathrm{NA}^{\mathrm{a}}(0 / 5)$ & $100 \%(5 / 5)$ \\
Chronic Demyelination & $0 \%(0 / 4)$ & $100 \%(4 / 4)$ & $100 \%(4 / 4)$ \\
Non-Affected Gray Matter & $0 \%(0 / 5)$ & $100 \%(5 / 5)$ & $100 \%(5 / 5)$ \\
\hline
\end{tabular}

6 clinical and pathologically confirmed multiple sclerosis autopsy samples were analyzed. Formalin-fixed paraffin-embedded $5 \mu \mathrm{m}$ thick sections were stained for H\&E, Luxol fast blue and periodic acid Schiff (LFB/PAS), and Bielschowsky's silver stain for routine pathology analysis. For cellular analysis, slides were stained for LRP1, myeloid marker CD68, and reactive astrocyte marker GFAP. Neurons were not detected in white matter

${ }^{a} N A$ not applicable 
Figure 8: LRP1 expression is increased in MS lesions

a

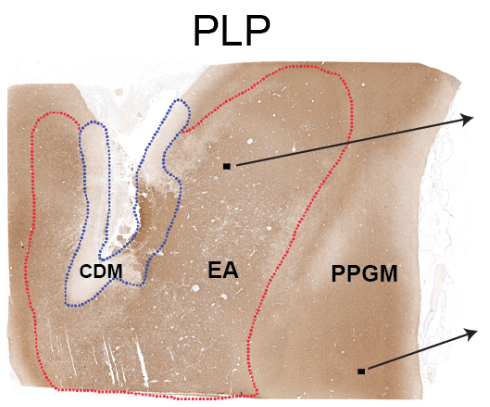

PLP

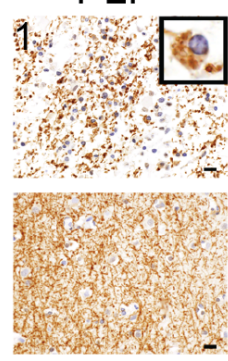

$\mathrm{CD68}$

GFAP

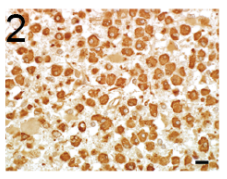

3
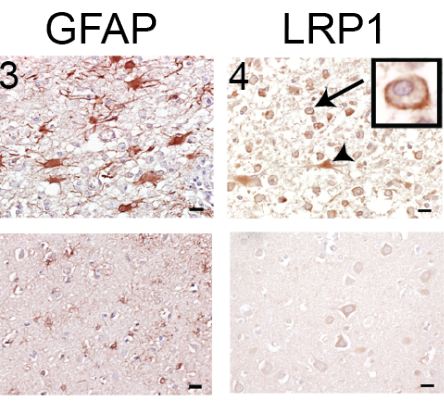

b

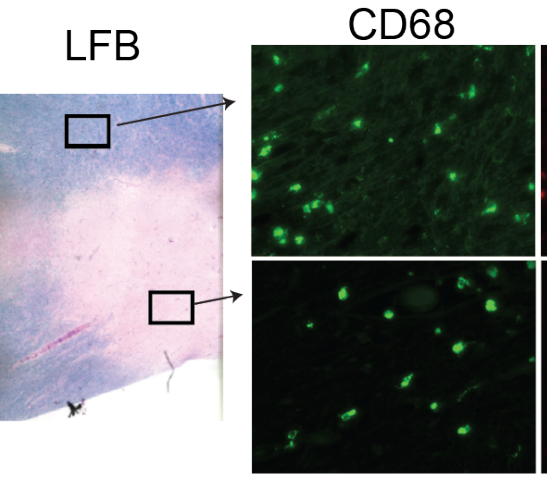

LRP1
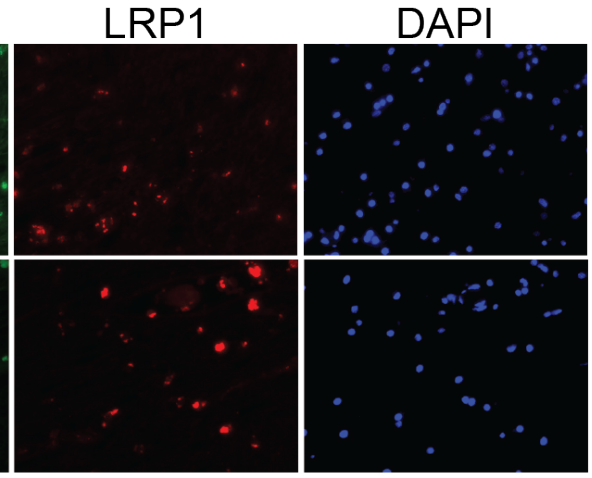

Merge

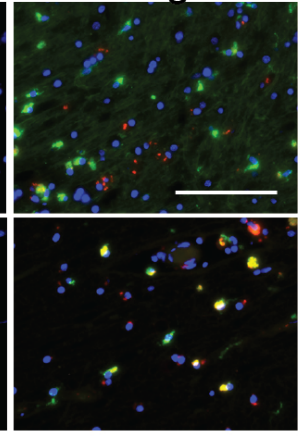

a Immunohistochemistry on consecutive sections from an early active (EA) MS lesion (upper row) highlights: (1) myelin (PLP) laden macrophages consistent with ongoing demyelinating activity, (2) abundant macrophage infiltration (CD68), (3) hypertrophic reactive astrocytes indicating gliosis (GFAP), and (4) LRP1 immunoreactivity present on both astrocytes (arrowhead) and macrophages (arrow and inset). In contrast, the periplaque gray matter (PPGM, lower row) shows: (1) normal appearing myelin (PLP), (2) limited microglial reactivity (CD68), (3) astrocytes with regular size and morphology (GFAP), and (4) limited LRP1 immunoreactivity. (Scale bars $=20 \mu \mathrm{m}$ ). b Luxol Fast Blue (LFB) histology and immunofluorescence for CD68 and LRP1 (Scale bar=100 $\mu \mathrm{m}$ ). 
Figure 9: LRP1 is deleted from microglia of the novel mouse strain Cx3cr $1^{\text {creER }}-L R P I^{f l f l}$, while in $\mathrm{Cx} 3 \mathrm{cr}^{1^{\text {cre }}-L R P 1^{f l t l}}$ mice, LRPI is deleted from both the microglia and the bone marrow-derived macrophages.

$C x 3 c r 1^{\text {creER }}-L R P I^{\text {fl/fl }} \quad$ and $C \times 3 c r I^{\text {cre }}-L R P I^{\mathrm{fl} / \mathrm{fl}}$ mice were perfused, and their CNS harvested, digested, and cleared of myelin by percoll gradient for flow cytometry analysis. The tibae and femurs were also removed from these mice and the bone marrow progenitor cells cultured in L929 media for 6 days to grow bone marrow macrophages. a Flow cytometry gating for microglia from the live singlet population, by $\mathrm{CD} 11 \mathrm{~b} / \mathrm{CD} 45$ staining, from $C x 3 c r l^{\text {creER }}$. $L R P I^{\mathrm{fl} / \mathrm{fl}}$ mice. b Quantification of the number of microglia in the brain and spinal cord of $C \times 3 c r 1^{\text {creER }}-L R P 1^{\mathrm{fl} / \mathrm{fl}}$ mice. c LRP1 expression on microglia
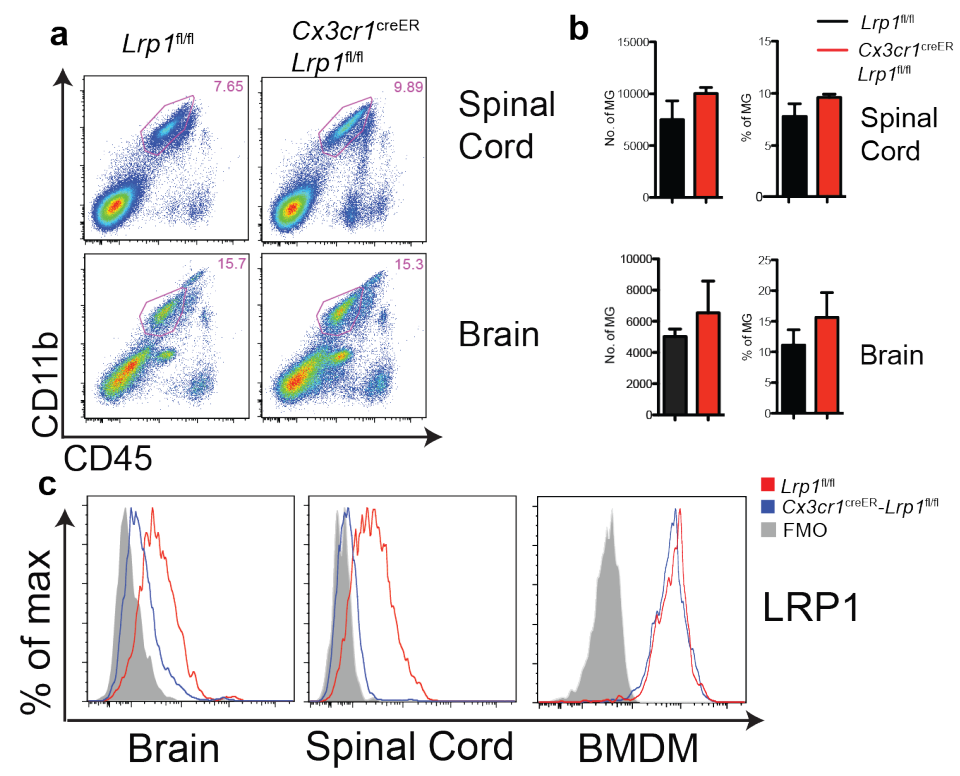

d

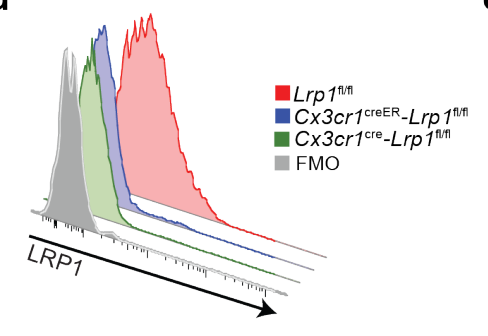

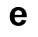

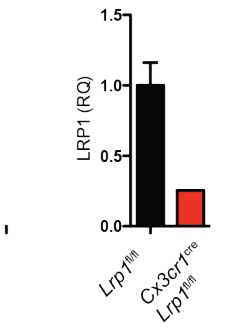

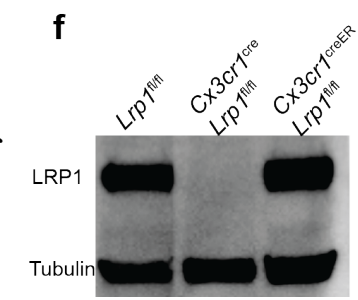

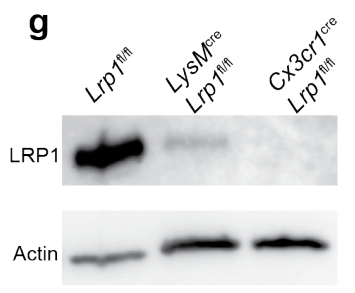

and bone marrow macrophages from $C x 3 c r 1^{\text {creER }}-L R P 1^{\mathrm{fl} / \mathrm{fl}}$ mice. $\mathbf{d}$ LRP1 expression on microglia from $C x 3 c r 1^{\text {creER }}-L R P 1^{\mathrm{fl} / \mathrm{fl}}$ mice and $C x 3 c r 1^{\text {cre }}-L R P 1^{\mathrm{fl} / \mathrm{fl}}$ mice. e LRP1 mRNA expression by qPCR from $C x 3 c r 1^{\text {cre }}-L R P 1^{\text {fl/fl }}$ macrophages. f Immunoblot of LRP1 expression from bone marrow derived macrophages from $C x 3 c r 1^{\text {creER }}-L R P 1^{\mathrm{fl} / \mathrm{ll}}$ mice mice and $C x 3 c r I^{\text {cre }}-L R P I^{\mathrm{fl} / \mathrm{fl}}$ mice mice. $\mathrm{g}$ Immunoblot of LRP1 expression from bone marrow derived macrophages from $C x 3 c r 1^{\text {creER }}-L R P I^{\mathrm{fl} / \mathrm{ll}}$ mice and $L y s M^{\mathrm{cre}}-L R P 1^{\mathrm{fl} / \mathrm{fl}}$ mice. 
Figure 10: Deletion of LRP1 from microglia, but not from peripheral myeloid cells, exacerbates EAE progression.
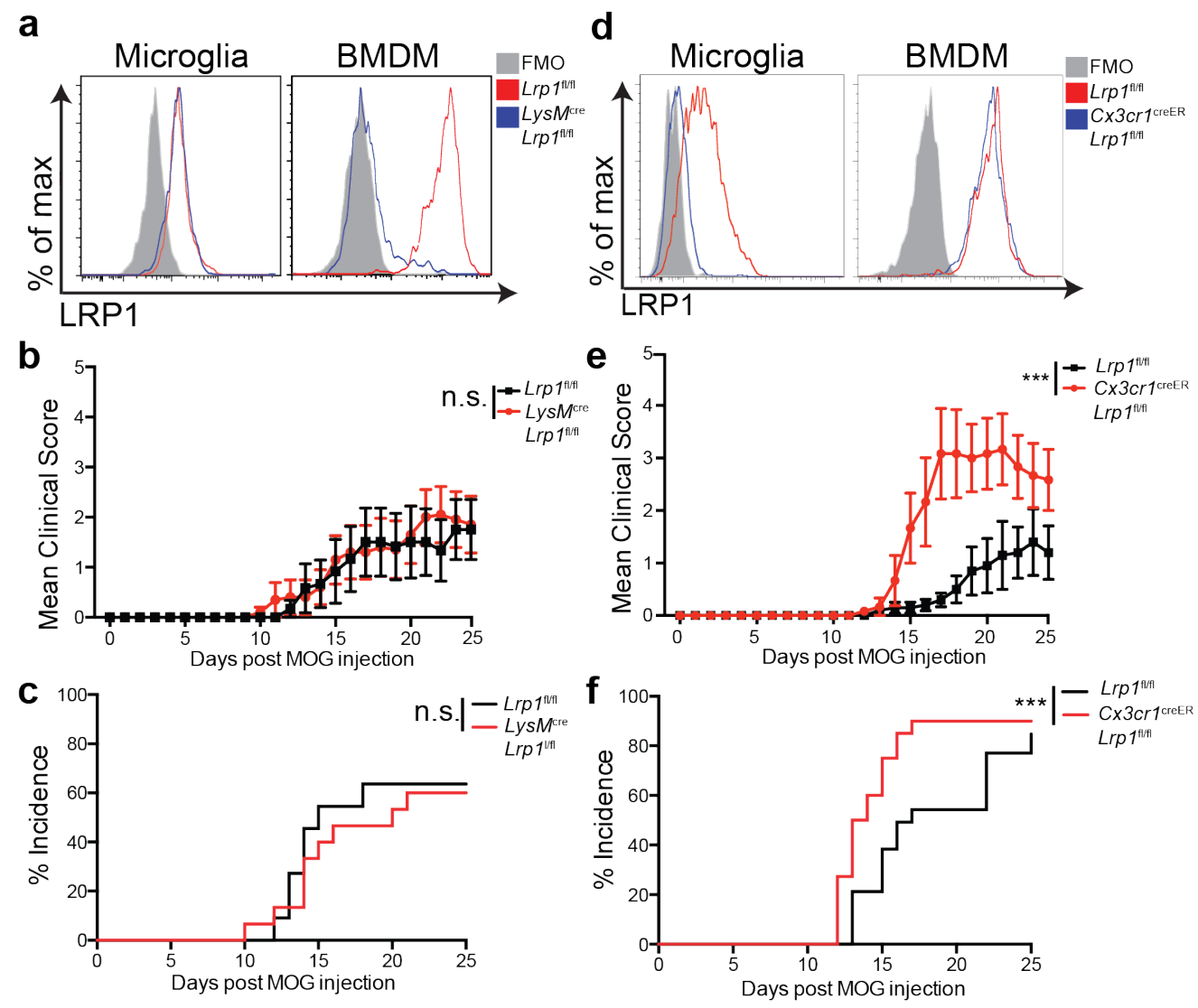

a LRP1 expression was determined by flow cytometry from microglia and BMDM isolated from $L y s M^{\mathrm{cre}}-\operatorname{Lrp} I^{\mathrm{fl} / \mathrm{fl}}$ and $\operatorname{Lrp} 1^{\mathrm{fl} / \mathrm{fl}}$ mice. b Clinical scores and $\mathbf{c}$ incidence of $L y s M^{\text {cre }}-\operatorname{Lrp} 1^{\mathrm{fl} / \mathrm{fl}}$ and $\operatorname{Lrp} 1^{\mathrm{fl} / \mathrm{fl}}$ mice (n=7-9 for each group, representative of 3 independent experiments). d LRP1 expression was determined by flow cytometry from microglia and BMDM isolated from $C x 3 c r 1^{\text {creER }}-\operatorname{Lrp} 1^{\mathrm{fl} / \mathrm{fl}}$ and $\operatorname{Lrp} 1^{\mathrm{fl} / \mathrm{fl}}$ mice. e Clinical scores and $\mathbf{f}$ incidence of $C x 3 c r 1^{\mathrm{creER}}-\operatorname{Lrp} 1^{\mathrm{fl} / \mathrm{fl}}$ and $\operatorname{Lrp} 1^{\mathrm{fl} / \mathrm{fl}}$ mice (n=6-10 for each group, representative of 3 independent experiments.) 2-way ANOVA was used for the mean clinical score and the log-rank (Mantle-Cox) test was used for incidence. $* * * p<0.001$, mean \pm s.e.m. 
Figure 11: Higher mycobacterium stimulus in LysM ${ }^{\text {cre }}-$ Lrp $^{\text {fllfl }}$ mice and CX3CR1

haplodeficiency do not affect EAE progression.

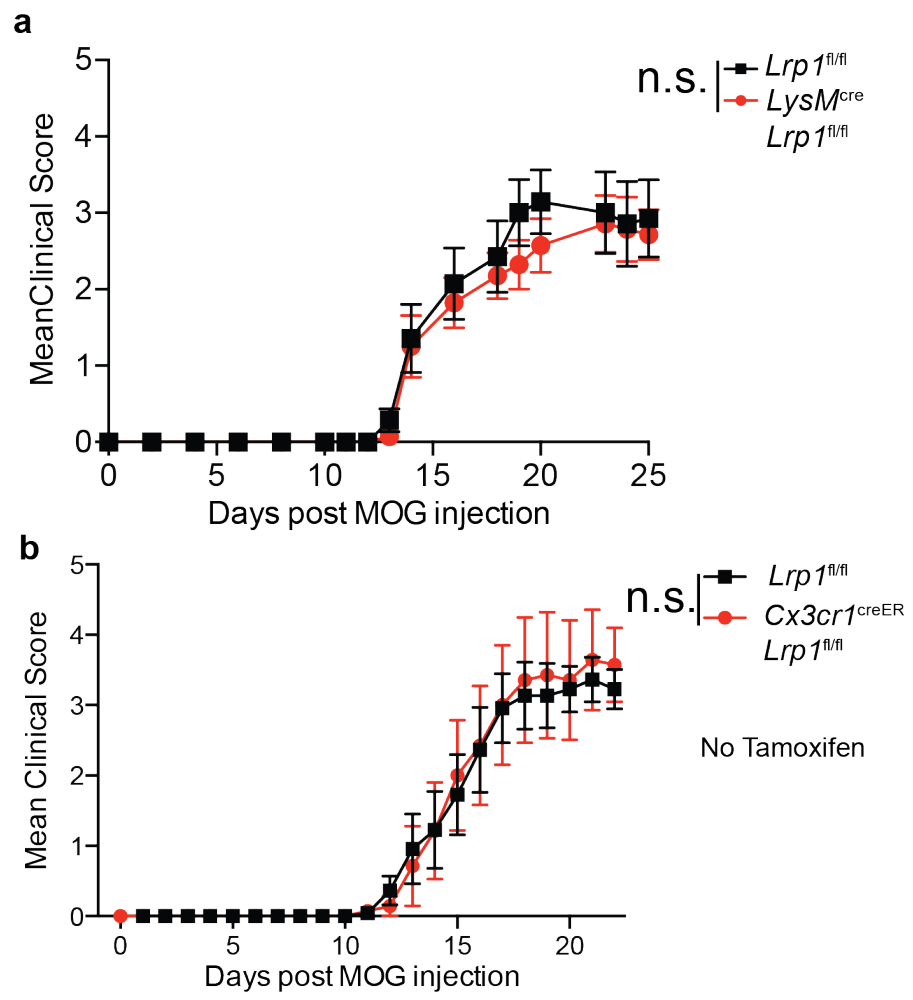

EAE was induced in a $L y s M^{\mathrm{cre}}-\operatorname{Lrp} 1^{\mathrm{fl} / \mathrm{fl}}$ and $\operatorname{Lrp} 1^{\mathrm{fl} / \mathrm{fl}}$ mice with a higher dose of mycobacterium $(2 \mathrm{mg} / \mathrm{ml})$ and in $\mathbf{b} C x 3 c r 1^{\text {creER }}-\operatorname{Lrp} 1^{\mathrm{fl} / \mathrm{fl}}$ and $\operatorname{Lrp} 1^{\mathrm{fl} / \mathrm{fl}}$ mice without tamoxifen injection. 2-way ANOVA, mean \pm s.e.m. 
Figure 12: Microglial LRP1 deficient mice have increased numbers of immune cells in the CNS during EAE.

Spinal cord sections of $C x 3 c r 1^{\text {creER }}$ $\operatorname{Lrp} 1^{\mathrm{fl} / \mathrm{fl}}$ and $\operatorname{Lrp} 1^{\mathrm{fl} / \mathrm{fl}}$ mice were stained with Luxol Fast Blue a at day $30(n=4$ for each group), and CD3 $\mathbf{b}(\mathrm{n}=4$ for each group) and IBA1 $\mathbf{c}(\mathrm{n}=4$ for each group) at day 15. Cells were manually quantified. For LFB, 2 sections per spinal cord level are plotted from each animal. For CD3 and Iba1, each point represents one animal. Immune cells were isolated by percoll gradient from spinal cords of $C x 3 c r 1^{\text {creER }}-L r p 1^{\mathrm{fl} / \mathrm{fl}}$ and $\operatorname{Lrp} 1^{\mathrm{fl} / \mathrm{fl}}$ mice. Number of $\mathbf{d}$ peripheral myeloid cells $\left(\mathrm{CD} 11 \mathrm{~b}^{\mathrm{hi}} \mathrm{CD} 45^{\mathrm{hi}}\right)$, e $\mathrm{T}$ cells $(\mathrm{TCR} \beta+)$ cells, f microglia $\left(\mathrm{CD} 11 \mathrm{~b}^{\mathrm{hi}} \mathrm{CD} 45^{\mathrm{mid}}\right)$, and $\mathrm{g} \mathrm{B}$ cells (CD19+) were determined by flow cytometry analysis $(n=3$ for each $\mathbf{f}$ group). ${ }^{*} \mathrm{p}<0.01, * * \mathrm{p}<0.001$; Student's
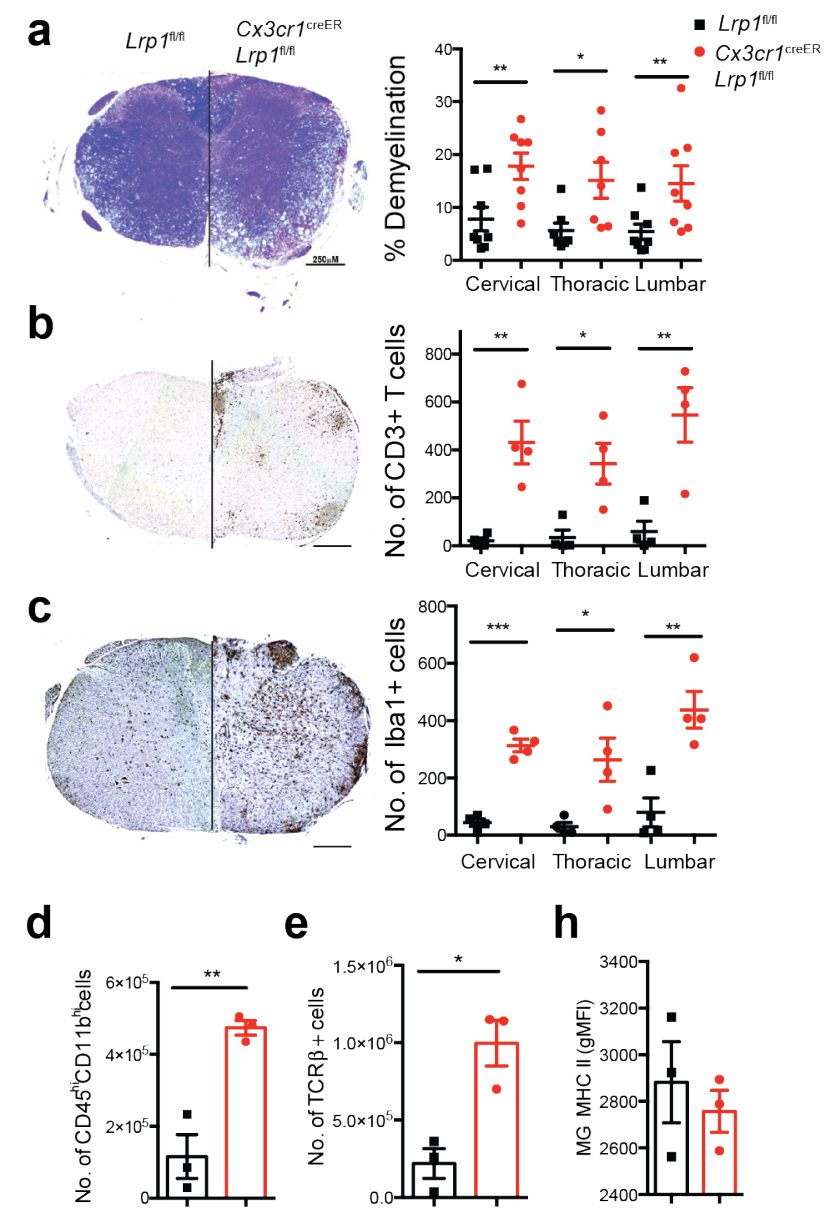
t-test, mean \pm s.e.m.

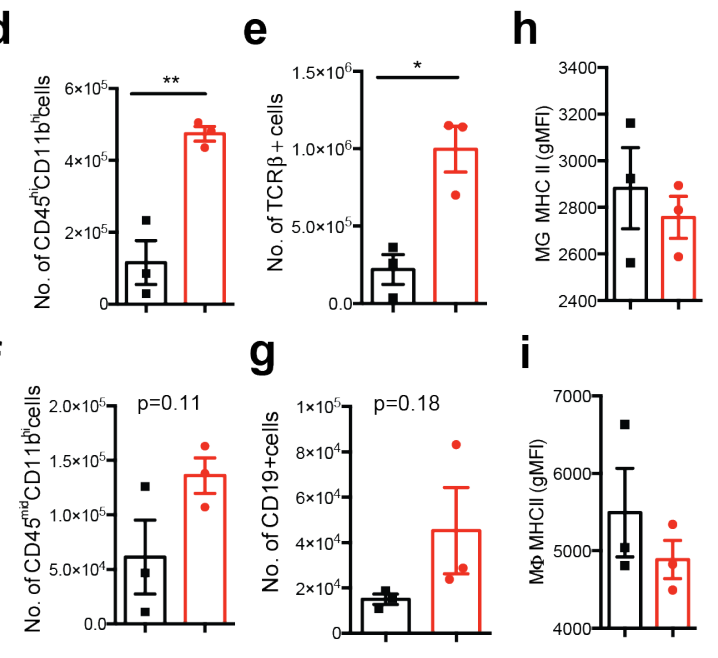


Figure 13: Immune response and blood-brain barrier permeability are not altered in

Microglial LRPI deficient mice.
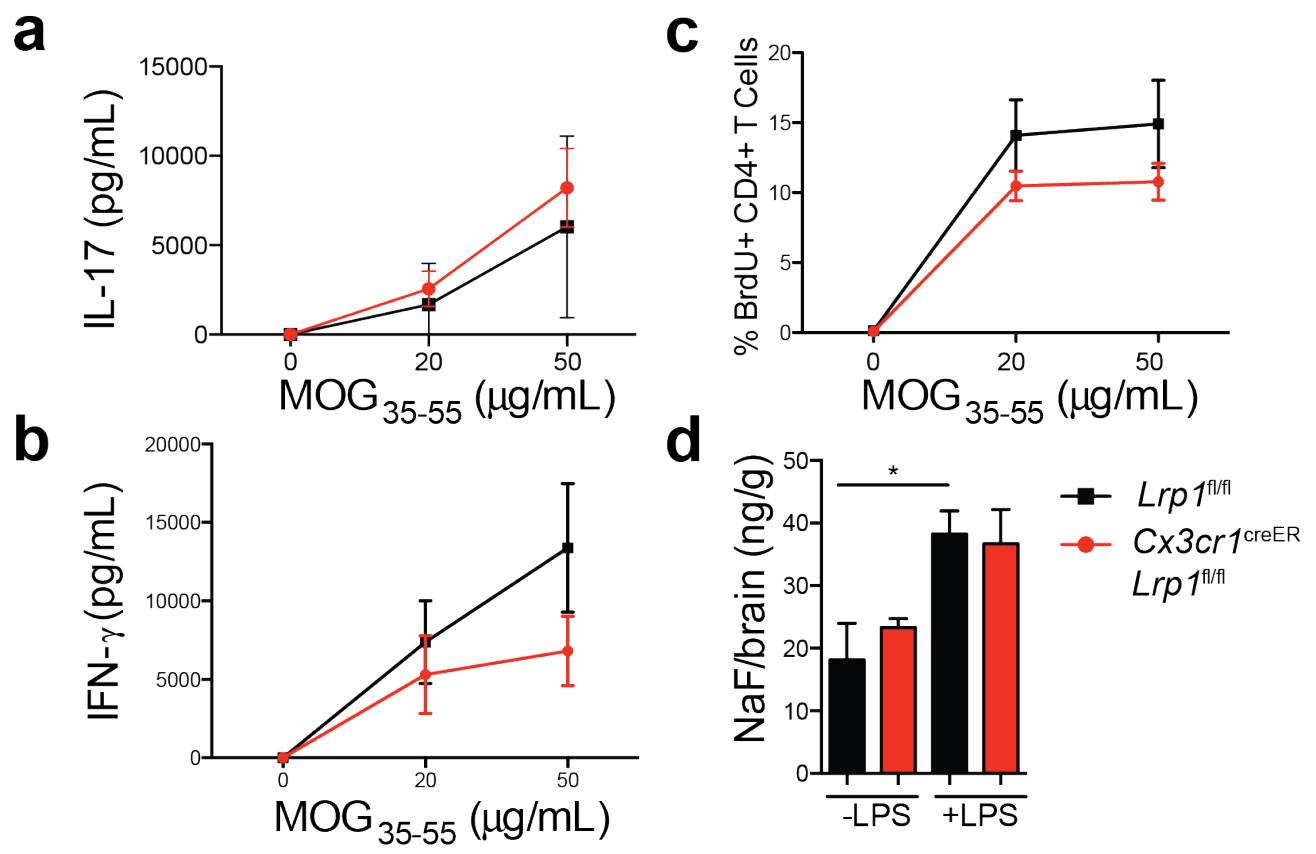

7 days after MOG immunization, draining lymph nodes were isolated from $C x 3 c r 1^{\text {creER }}$ $\operatorname{Lrp} 1^{\mathrm{fl} / \mathrm{fl}}$ and $\operatorname{Lrp} 1^{\mathrm{fl} / \mathrm{fl}}$ mice and incubated with MOG. a-b IFN- $\gamma$ and IL-17A production were determined by ELISA after 24h. c BrdU incorporation by $\mathrm{T}$ cells was determined after $16 \mathrm{~h}$ by flow cytometry ( $\mathrm{n}=3-5$ mice were used per group per experiment). 2-way ANOVA, mean \pm s.e.m. d The amount of fluorescein present in brains following LPS treatment $\left(6 \mathrm{mg} / \mathrm{kg}\right.$ for 6 hours) was measured in $C x 3 c r 1^{\text {creER }}-\operatorname{Lrp} 1^{\mathrm{fl} / \mathrm{fl}}$ and $\operatorname{Lrp} 1^{\mathrm{fl} / \mathrm{fl}}$ mice to assess function of the blood brain barrier. Student's t-Test, mean \pm s.e.m. 
Figure 14: LRP1 deficient microglia have an amoeboid morphology.

a

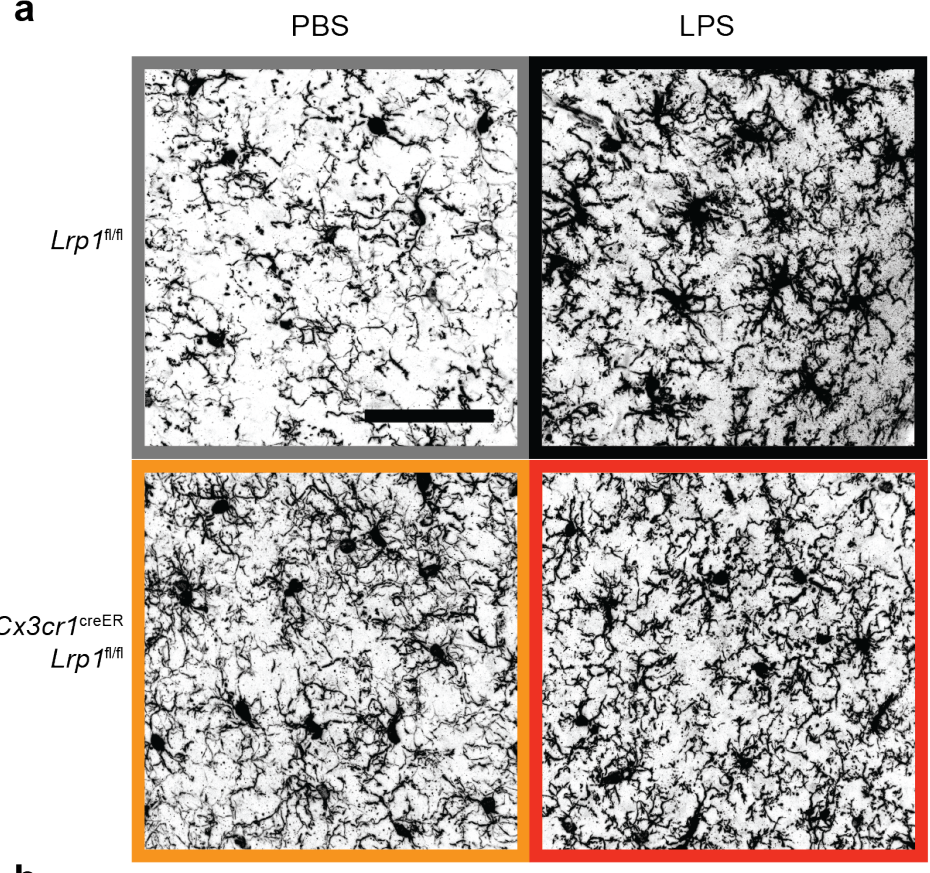

b

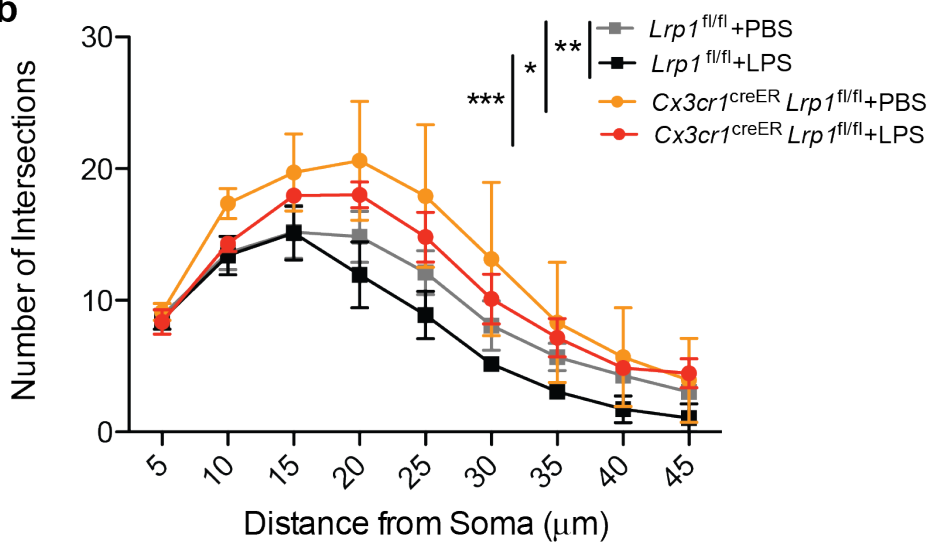

C

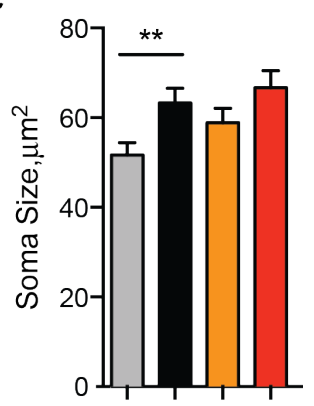

d

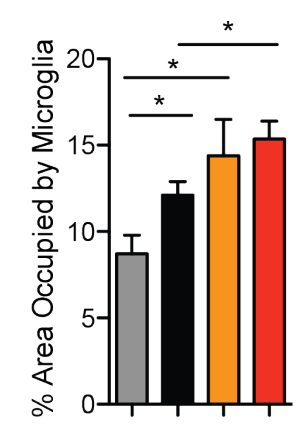

e

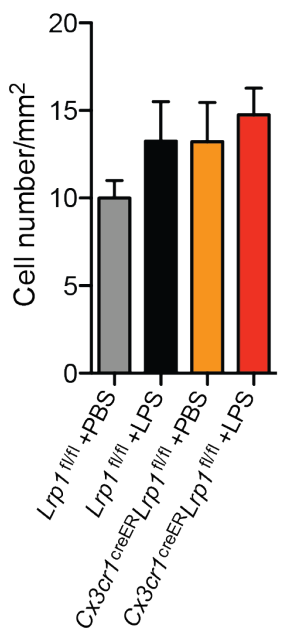

$C x 3 c r 1^{\text {creER }}-L r p 1^{\mathrm{fl} / \mathrm{fl}}$ and $L r p 1^{\mathrm{fl} / \mathrm{fl}}$ mice were injected daily with LPS $(1 \mathrm{mg} / \mathrm{kg})$ for 4 consecutive days. Brain sections were stained with IBA1 antibody, imaged by confocal microscopy and analyzed with ImageJ. a Representative images of microglia and corresponding Sholl analyses. ${ }^{*} \mathrm{p}<0.1 ;{ }^{*} \mathrm{p}<0.01$; ${ }^{* * *} \mathrm{p}<0.001,2$-way ANOVA. (Scale bar $=60 \mu \mathrm{m})$. b Quantification of area covered by IBA1+ microglia per image. ${ }^{*} \mathrm{p}<0.1$, Student's t-test. c Numbers of microglia per unit area (3-4 mice per condition). d Soma size of the microglia. 10 microglia per animal were quantified for each experimental group. ${ }^{*} \mathrm{p}<0.01$, Student's t-test; mean \pm s.e.m. 
Figure 15: LRP1 deficiency in microglia does not alter weight loss after LPS treatment.

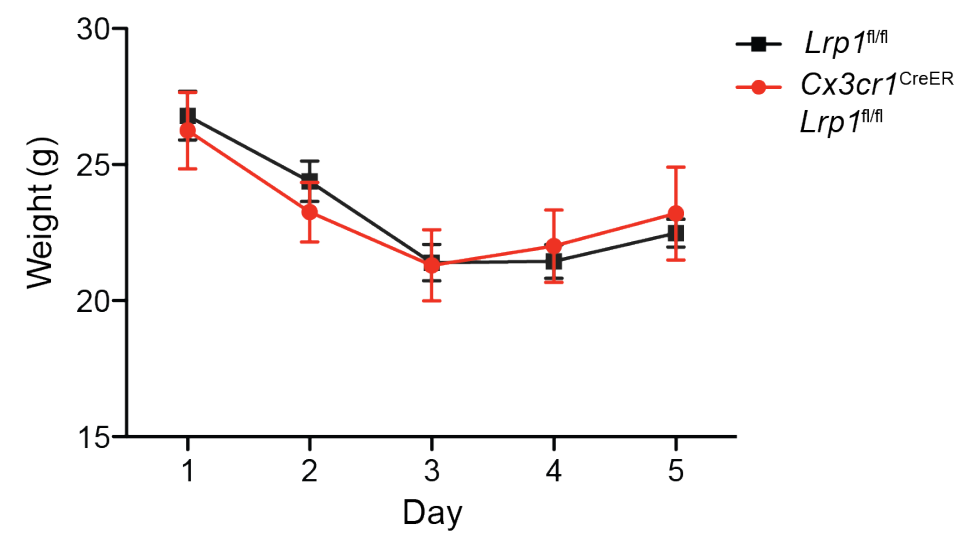

$C x 3 c r 1^{\text {creER }}-L r p 1^{\mathrm{fl} / \mathrm{fl}}$ and $\operatorname{Lrp} 1^{\mathrm{fl} / \mathrm{fl}}$ mice were injected daily with LPS $(1 \mathrm{mg} / \mathrm{kg})$ for 4 consecutive days and weight of the animals was monitored daily. 2-way ANOVA; mean \pm s.e.m. 
Figure 16: LRP1 deficient myeloid cells have a pro-inflammatory signature.

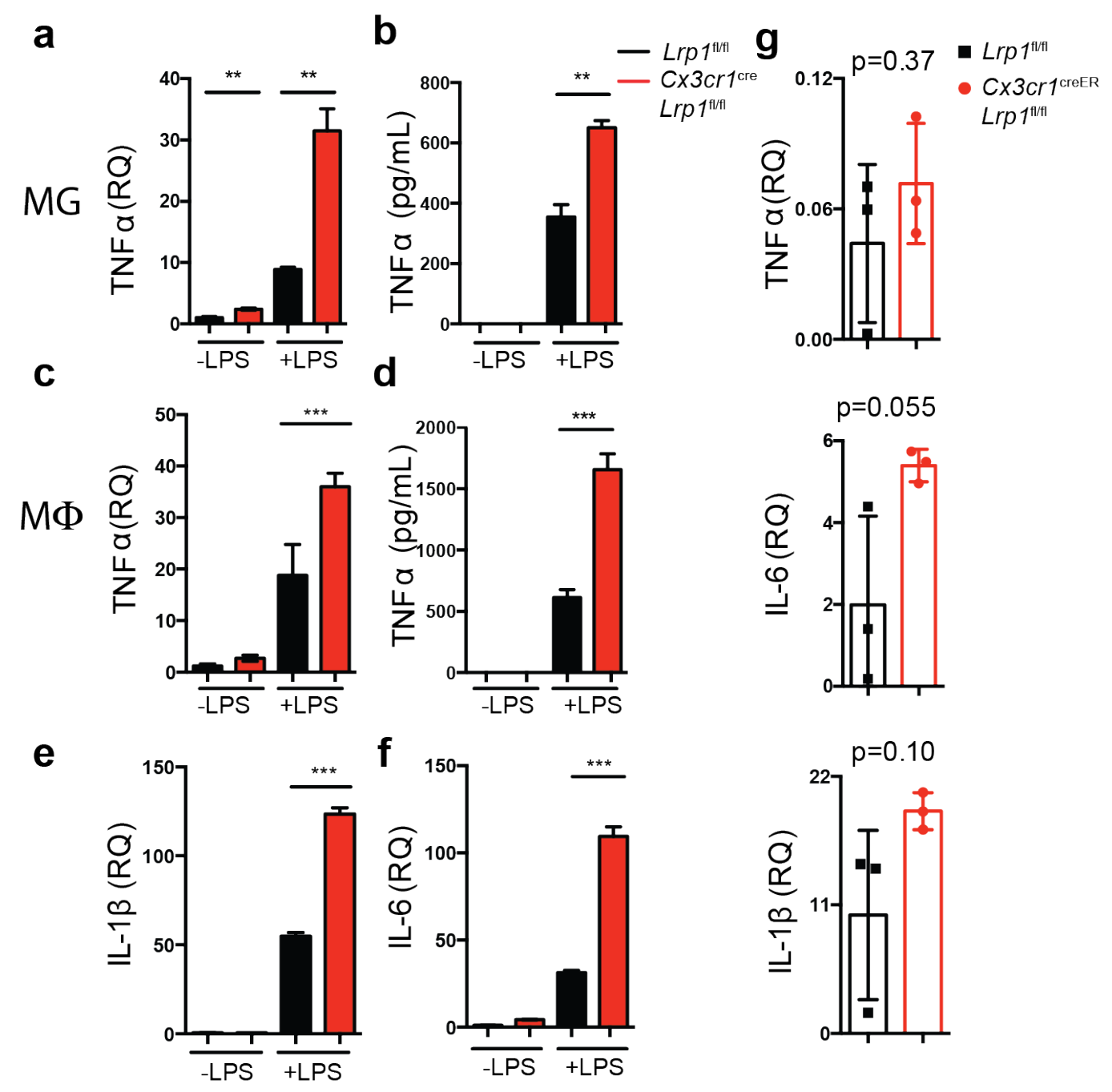

Microglia (MG) and BMDM (MФ) isolated from $C x 3 c r 1^{\text {cre }}-L r p 1^{\mathrm{fl} / \mathrm{fl}}$ and $L r p 1^{\mathrm{fl} / \mathrm{fl}}$ mice were treated with LPS $(1 \mu \mathrm{g} / \mathrm{ml})$ for 3 hours (qPCR) or 24 hours (ELISA). TNF $\alpha$ expression in microglia was determined by $\mathbf{a} \mathrm{qPCR}$ and $\mathbf{b}$ ELISA. TNF $\alpha$ expression in macrophages was determined by $\mathbf{c}$ qPCR and $\mathbf{d}$ ELISA. Expression of $\mathbf{e}$ IL- $1 \beta$ and $\mathbf{f}$ IL- 6 in macrophages was determined by qPCR. $\mathbf{g}$ Transcript expression of IL-1 $\beta$, IL-6, and TNF- $\alpha$ from the CNS of $C x 3 c r 1^{\text {creER }}-L_{r p} I^{\mathrm{fl} / \mathrm{fl}}$ and $\operatorname{Lrp} 1^{\mathrm{fl} / \mathrm{fl}}$ mice at the peak of EAE. ${ }^{*} \mathrm{p}<0.01,{ }^{* *} \mathrm{p}<0.001,{ }^{* * *} \mathrm{p}<0.001$; Student's t-test, mean \pm s.e.m. 
Figure 17: LRP1 deficient microglia have an increased pro-inflammatory signature in $E A E$
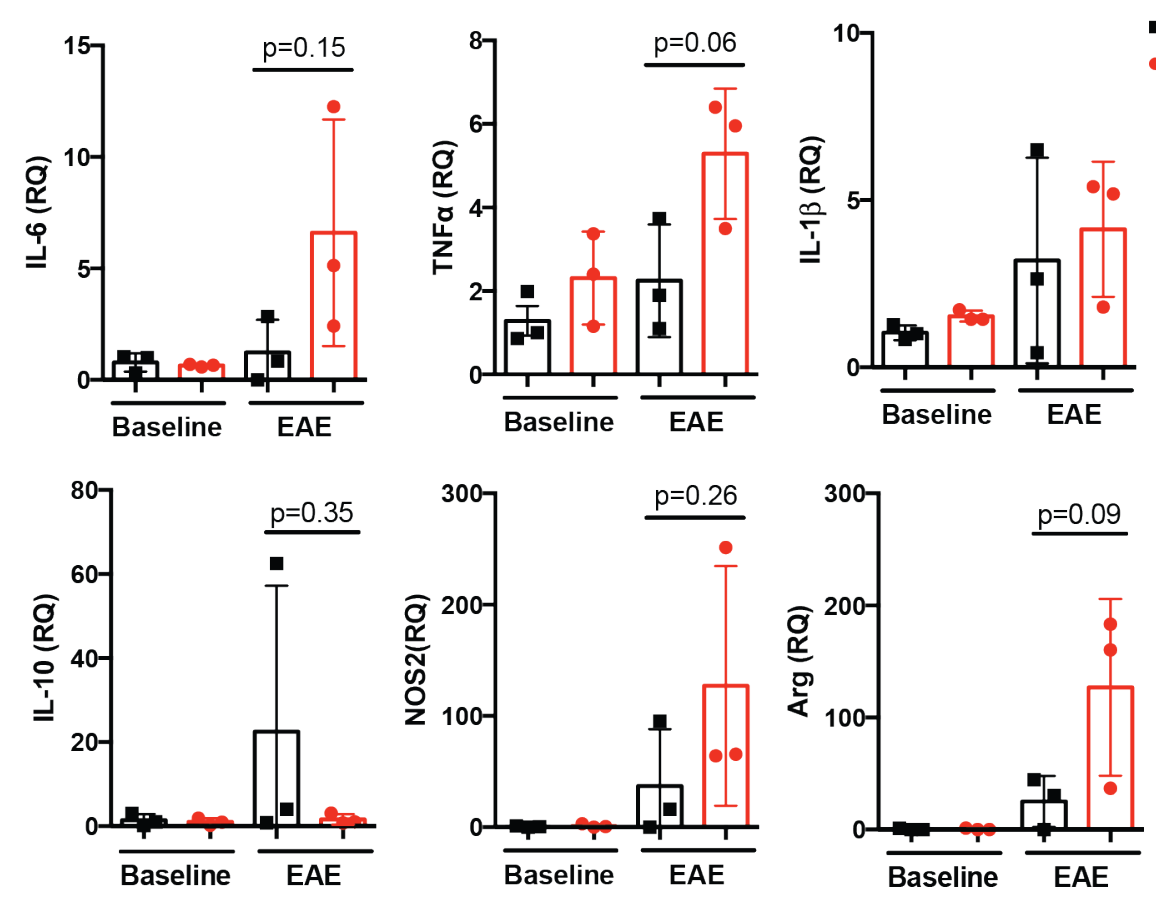

Microglia isolated by digestion and FACS sort were obtained from $C x 3 c r 1^{\mathrm{cre}}-\operatorname{Lrp} 1^{\mathrm{fl} / \mathrm{fl}}$ and $\operatorname{Lrpl} 1^{\mathrm{fl} / \mathrm{fl}}$ mice with and without EAE. Expression of a panel of pro-inflammatory and activation markers was determined by qPCR analysis. Student's t-test, mean \pm s.e.m. 
Figure 18: LRP1 is an inhibitor of NF-xB through the MyD88 pathway.

a

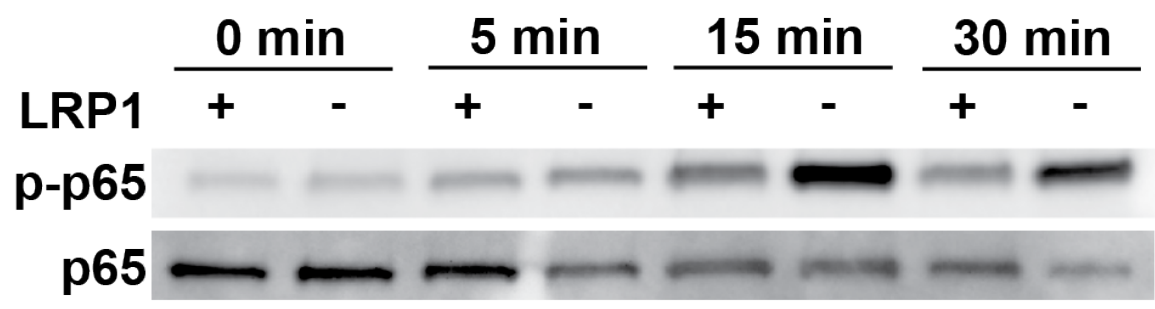

LRP1

Actin

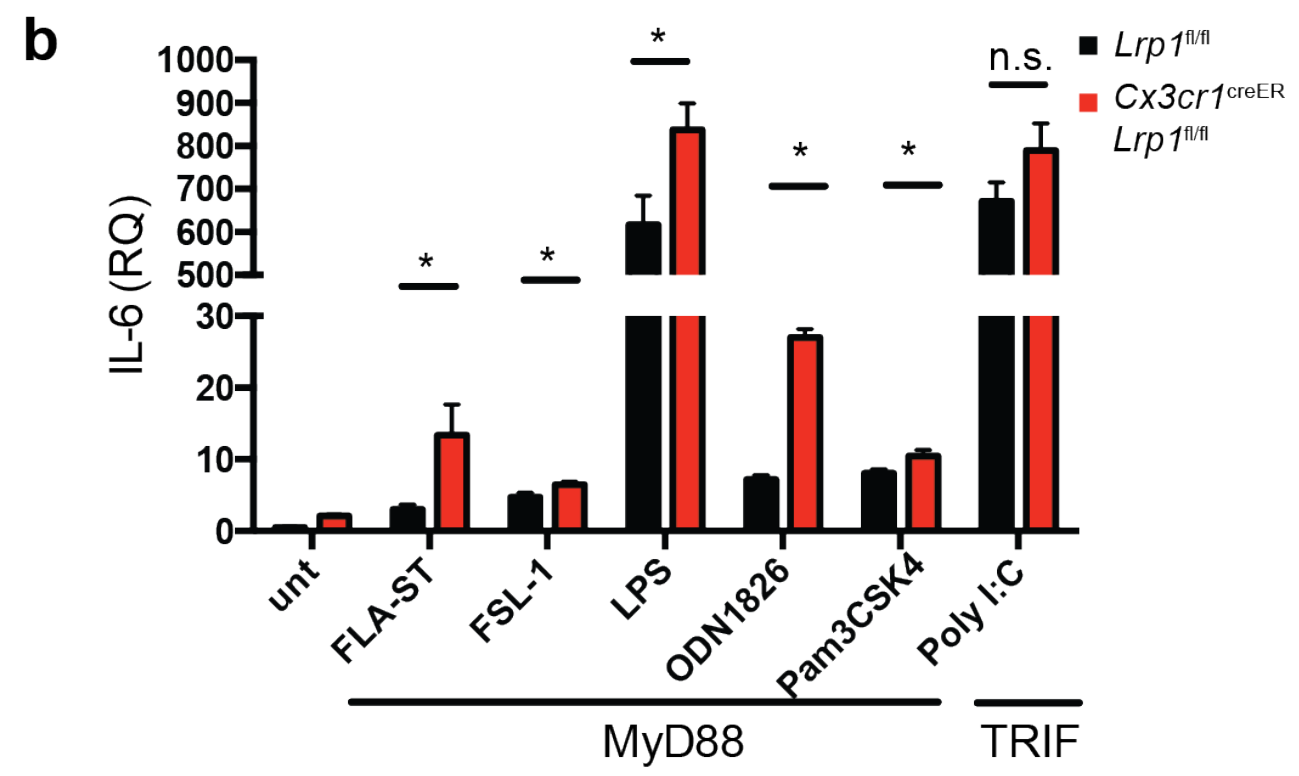

a Macrophages isolated from $C x 3 c r 1^{\text {cre }}-\operatorname{Lrpl}^{\mathrm{fl} / \mathrm{fl}}(\mathrm{LRP} 1-)$ and $\operatorname{Lrpl} 1^{\mathrm{fl} / \mathrm{fl}}(\mathrm{LRP} 1+)$ mice were treated with LPS $(1 \mu \mathrm{g} / \mathrm{ml})$ and the expression of p-p65, p65, LRP1 and actin were determined by immunoblot. (Data is representative of 3 independent experiments). b LRP1+ and LRP1-macrophages were treated with TLR ligands and expression of IL-6 was determined by qPCR analysis. (Data is representative of 2 independent experiments). ${ }^{*} \mathrm{p}<0.05 ;$ Student's t-test, mean \pm s.e.m. 


\title{
Chapter 4: LRP1 IS ESSENTIAL FOR THE PHAGOCYTOSIS OF MYELIN AND CELLULAR DEBRIS IN MS
}

\begin{abstract}
:
In the previous chapter, I have demonstrated that LRP1 was protective during EAE by an anti-inflammatory, MyD88 dependent mechanism. However, LRP1 is mostly understood in the context of its function as a scavenger receptor and the question of LRP1's role in phagocytosis during EAE was largely unexplored. Myeloid cells are instrumental phagocytes during EAE, and the phagocytosis of myelin and apoptotic cells induces an anti-inflammatory effect on myeloid cells. As there are copious amounts of debris generated during multiple sclerosis and other neurodegenerative diseases, it is important to determine the contribution of phagocytosis to the phenotype observed in animals lacking microglial LRP1. Therefore, I began to explore the role of LRP1 in the context of phagocytosis of debris using a combination of in vitro and in vivo models. This work will provide a foundation for a future publication regarding the role of LRP1 in mediating the crosstalk between phagocytosis and inflammation.
\end{abstract}

\section{Introduction}

LRP1 is a scavenger receptor known to bind over 100 different ligands ${ }^{120}$. Normally present at the cell surface, LRP1 consists of an extracellular, 500kD $\alpha$-chain and a transmembrane, $75 \mathrm{kD}$ intracellular $\beta$-chain. Upon recognition of its ligands, LRP1 initiates clathrin-mediated endocytosis to deliver its cargo to the endosome and eventually, the lysosome. LRP1 is then recycled from this pathway back to the cell 
surface. LRP1 is known to bind, among other ligands, apoptotic and necrotic cell debris $^{133}$. Myeloid cells, particularly macrophages, are instrumental in the clearance of debris to maintain homeostasis. Beyond hepatocytes, LRP1 is most highly expressed by myeloid cells within the immune cell types ${ }^{175}$.

Multiple Sclerosis (MS) is the most common CNS demyelinating disorder. MS is characterized by the death of myelin sheaths that insulate neurons, resulting in diffuse CNS symptoms and inflammation ${ }^{5}$. The increase in myelin and apoptotic debris within a demyelinating MS lesion requires phagocytic clearance, which is largely mediated by myeloid cells such as macrophages. Within the context of MS, multiple groups have implicated microglia in the pathogenesis of disease ${ }^{63,159}$. Immunohistochemistry for CD68+ myeloid cells - which include microglia and peripherally derived macrophages have shown that there are an increased number of these cells during MS. Furthermore, microglial activation occurs before disease onset in EAE, a mouse model of MS. The resident microglia are known to regularly interact with their environment by dynamically extending and retracting their processes ${ }^{60,61}$. Microglial sensing of environmental change and neuronal activity affords them the ability to prune neurons in an activity dependent fashion $^{57}$. Thus, microglia are sensitive to environmental cues and change their phagocytic behavior accordingly.

Our lab has previously described the role of LRP1 as a phagocytic receptor for myelin and necrotic cell debris. By mRNA analysis, LRP1 is highly expressed on microglia, at a thousand-fold that of peripheral blood monocytes ${ }^{65}$. Therefore, I hypothesize that LRP1 could play a more important role in phagocytosis in microglia than in peripheral myeloid cells. Modulating phagocytosis may also contribute to LRP1's 
previously described role in mediating an anti-inflammatory effect on microglia during $\mathrm{EAE}^{176}$.

\section{Results:}

LRP1 deficient myeloid cells have impaired myelin phagocytosis, but not of foreign molecules and bacteria

To examine whether LRP1 deficient myeloid cells have a measurable defect in phagocytosis, we examined the engulfment of $\mathrm{pH}$ sensitive fluorescently labeled myelin by LRP1 deficient microglia purified from $C x 3 c r 1^{\text {creER }}-L r p 1^{\mathrm{fl} / \mathrm{fl}}$ mice (Fig. 19a). This special labeling allows detection of the late stage of phagocytosis, when the cargo becomes acidified in the lysosomal compartment. LRP1 deficient microglia show lack of myelin uptake, whereas control microglia begin to show significant increase in lysosomal myelin content at 45 minutes after treatment. To verify whether this defect was specific to myelin or extends to other ligands such as bacterial and yeast peptides, I administered $\mathrm{pH}$ sensitive fluorescently labeled zymosan and E. coli to LRP1 deficient bone marrowderived macrophages. The LRP1 expressing and LRP1 deficient macrophages have no different in uptake of fluorescently labeled peptides originating from foreign organisms (Fig. 19b). These results indicate that the phagocytic deficiency of LRP1 myeloid cells is specific to LRP1 ligands such as myelin. Furthermore, it suggests that the difference in the LPS inflammatory response in LRP1 deficient myeloid cells could originate from a mechanism independent of phagocytosis. 
During EAE, LRPI deficient microglia may have lysosomal deficits related to phagocytosis

To test if LRP1 could participate in the removal of myelin in vivo, I analyzed the mRNA expression of lysosomal pathway genes from FACS purified peripheral myeloid cells $\left(\mathrm{CD} 45^{\mathrm{hi}} \mathrm{CD} 11 \mathrm{~b}^{\mathrm{hi}}\right)$ and microglia $\left(\mathrm{CD} 45^{\mathrm{mid}} \mathrm{CD} 11 \mathrm{~b}^{\mathrm{hi}}\right)$ from our $C x 3 c r 1^{\mathrm{creER}}-\operatorname{Lrp} 1^{\mathrm{fl} / \mathrm{fl}}$ mice during onset of disease in EAE (Fig. 20). In the microglia (MG) population, there is a trend towards altered mRNA expression of certain genes involved in the lysosomal pathway. There seems to be a decrease in TFEB and CLCN7, and an increase in ATP6V1H expression in the LRP1 deficient microglia. As a comparison, the expression of these genes was not noticeably altered in the peripheral monocyte-derived macrophage population. Other targets of lysosomal activity, namely CTSA, HEXA, and LAMP1, were not affected in LRP1 deficient microglia. TFEB was identified as a master regulator of lysosomal function and response to changes in phagocytosis ${ }^{92}$. Taken together, this data suggests that there may be an alteration in lysosomal generation in the LRP1 deficient microglial population during EAE, pointing toward a defective phagocytic pathway.

$C \times 3 c 1^{\text {cre }}{ }^{2} P P I^{f l f l}$ mice have deficits in oligodendrocyte proliferation in a cuprizone model of demyelination

Another key function of myelin phagocytosis is to promote tissue repair. Indeed, failure to clear myelin has been shown to block remyelination in a mouse model of primary demyelination ${ }^{95}$. In order to examine the role of microglial LRP1 on phagocytic clearance in vivo, I maintained $C x 3 c r 1^{\mathrm{creER}}-\operatorname{Lrp} 1^{\mathrm{fl} / \mathrm{fl}}$ and $\operatorname{Lrp} 1^{\mathrm{fl} / \mathrm{fl}}$ control mice on a 
cuprizone diet for 5 weeks. Cuprizone specifically targets oligodendrocytes for apoptotic death and the demyelination can be assessed in the corpus callosum.

Quantification of images of the corpus callosum stained with CC1 (green), a mature oligodendrocyte marker, and Olig2 (red), an oligodendrocyte lineage marker (Fig. $21 \mathrm{a}-\mathrm{b})$, reveal that while there was no difference in the overall number of cells of the oligodendrocyte lineage (Fig. 21c), there is a trend towards a decreased number of oligodendrocytes progenitor cells (OPC) in the corpus callosum and an increased number of oligodendrocytes (OLG) (Fig. 21d) in the microglial LRP1 deficient mice. These preliminary data suggest that in the absence of LRP1 expression on microglia, the population of OPCs is affected during cuprizone-induced demyelination. Further work is needed to understand the potential function of LRP1 in myeloid cells in the context of remyelination.

\section{Discussion}

The function of LRP1 in the removal of degraded myelin and dying cells could be critical in maintaining CNS homeostasis under normal physiological conditions, as well as in removing the degenerating oligodendrocytes during MS ${ }^{131,133}$. Thus, I tested the phagocytic ability of LRP1 deficient myeloid cells and found that while they are deficient in myelin engulfment, they are not deficient in engulfment of TLR2 (yeast) nor TLR4 (bacterial) ligands. Since LRP1 affects the phagocytosis of myelin, we isolated microglia and macrophages purified during EAE from microglial LRP1 deficient mice and analyzed lysosomal genes known to be upregulated during phagocytosis. These studies revealed that the microglia lacking LRP1 might have a defect in phagocytic regulation, 
while the macrophages remain unaffected, suggesting that LRP1 may be important during the increase in myeloid phagocytic activity of EAE.

Proper clearance of the myelin debris is critical for brain function, as the release of degenerated myelin could further propagate inflammation and damage the CNS. Furthermore, myelin-laden macrophages isolated from the brain are known to have decreased inflammatory responses, when compared to macrophages that have not engulfed myelin ${ }^{96}$. The receptors and mechanisms that initiate the anti-inflammatory response to apoptotic cell or myelin engulfment are still poorly understood ${ }^{56}$. Since LRP1 is heavily involved in phagocytic processes of the cell, it is a potential candidate for simultaneous regulation of the cellular phagocytic and inflammatory responses. To investigate potential roles of microglial LRP1 on the remyelination process, we induced demyelination using the cuprizone model. Immunofluorescence reveals that there is no overall change in the number of cells of the oligodendrocyte lineage (Olig2+ cells), but there is a trend toward decreased proportion of OPC. Future studies into the extent of demyelination by examining the degraded myelin basic protein would implicate microglial LRP1 in the phagocytosis of myelin debris. Furthermore, analysis of the OPC cell fates - whether this decrease in proportion of OPC is due to decreased apoptosis, or increased differentiation of oligodendrocytes - in the microglial LRP1 deficient mice would illuminate the role of LRP1 during primary demyelination. These analyses would inform whether LRP1 mediated phagocytosis in microglia could initiate an antiinflammatory response, maintain oligodendrocyte health, and perhaps be protective during the disease process in MS. 


\section{Figures}

Figure 19: LRP1 deficient myeloid cells are deficient in phagocytosis of myelin, but not

$$
\text { of TLR ligands }
$$

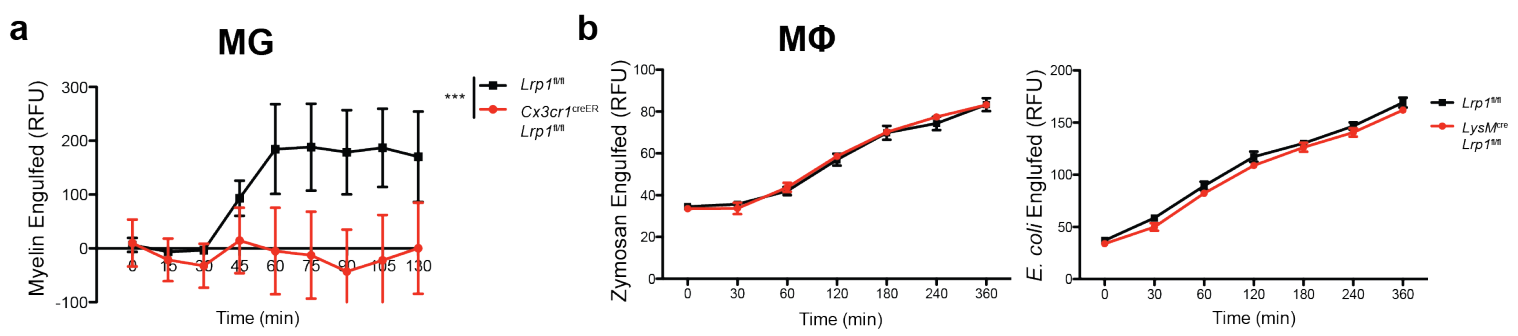

LRP1 deficient microglia $\left(C x 3 c r 1^{\text {creER }}-L r p 1^{\mathrm{fl} / \mathrm{fl}}\right)$ and macrophages $\left(L y s M^{\mathrm{cre}}-\operatorname{Lrp} 1^{\mathrm{fl} / \mathrm{fl}}\right)$ were isolated and plated into 96 well black walled plates with clear bottoms. a LRP1 deficient microglia were treated with fluorescently labeled myelin (CypHer5e), while b LRP1 deficient macrophages were treated with zymosan or E. coli (pHrodo), and the fluorescence measured over time. ${ }^{* * *} p<0.001$, 2-way ANOVA; mean \pm s.e.m.. $\mathbf{a}$ is representative of one independent experiment, while $\mathbf{b}$ is representative of 3 independent experiments. 
Figure 20: LRP1 deficient microglia have altered lysosomal gene regulation
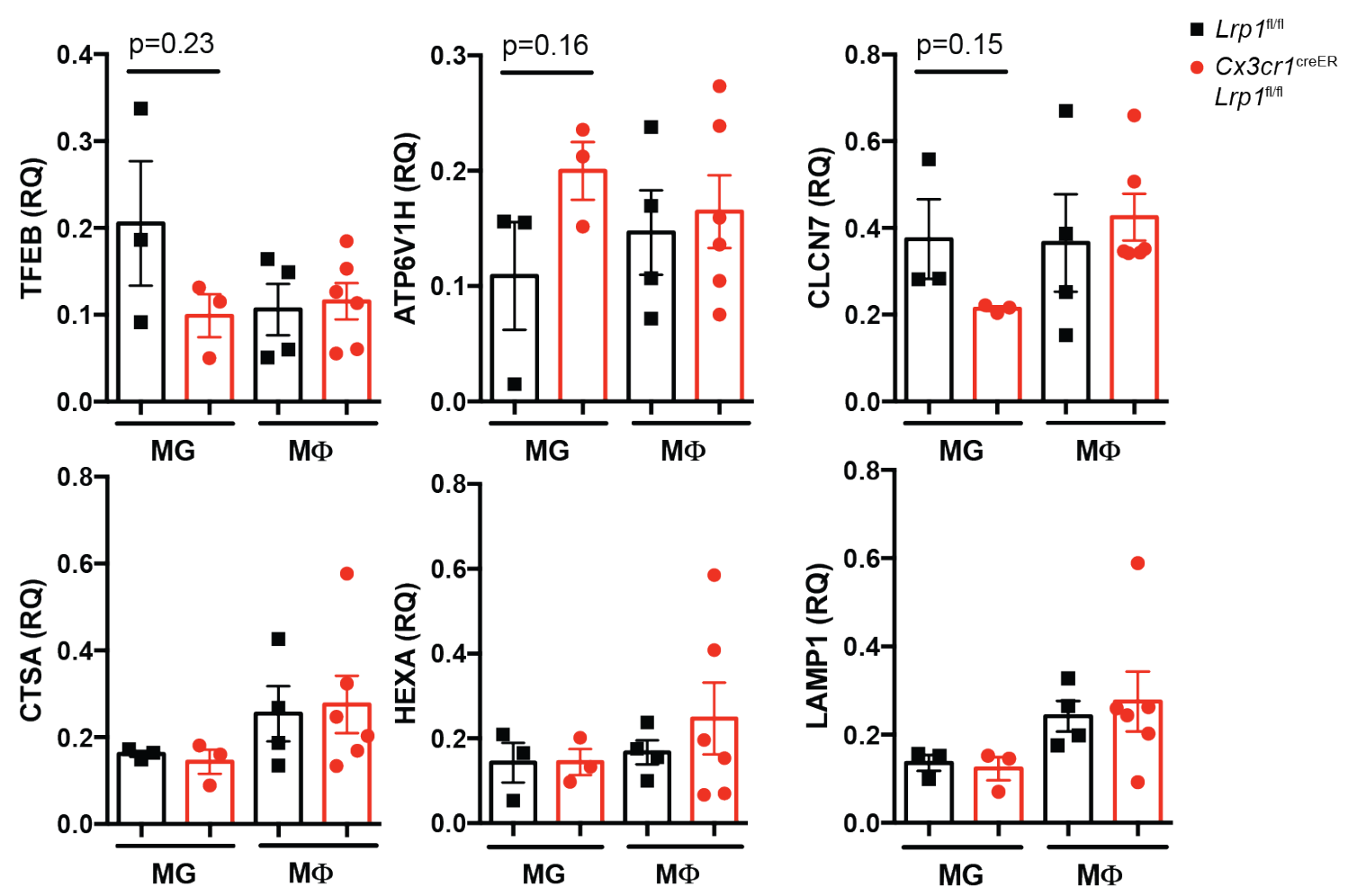

Microglia $\left(\mathrm{CD} 11 \mathrm{~b}^{\mathrm{hi}} \mathrm{CD} 45^{\mathrm{mid}}\right)$ and macrophages $\left(\mathrm{CD} 11 \mathrm{~b}{ }^{\mathrm{hi}} \mathrm{CD} 45^{\mathrm{hi}}\right)$ were FACS sorted from the spinal cords of EAE mice ( $n=5$ per group), and gene expression involved in lysosomal biogenesis and regulation were determined by qPCR analysis. Student's tTest; mean \pm s.e.m. 
Figure 21: Mice with LRP1 deficient microglia have less oligodendrocyte progenitor cells during demyelination
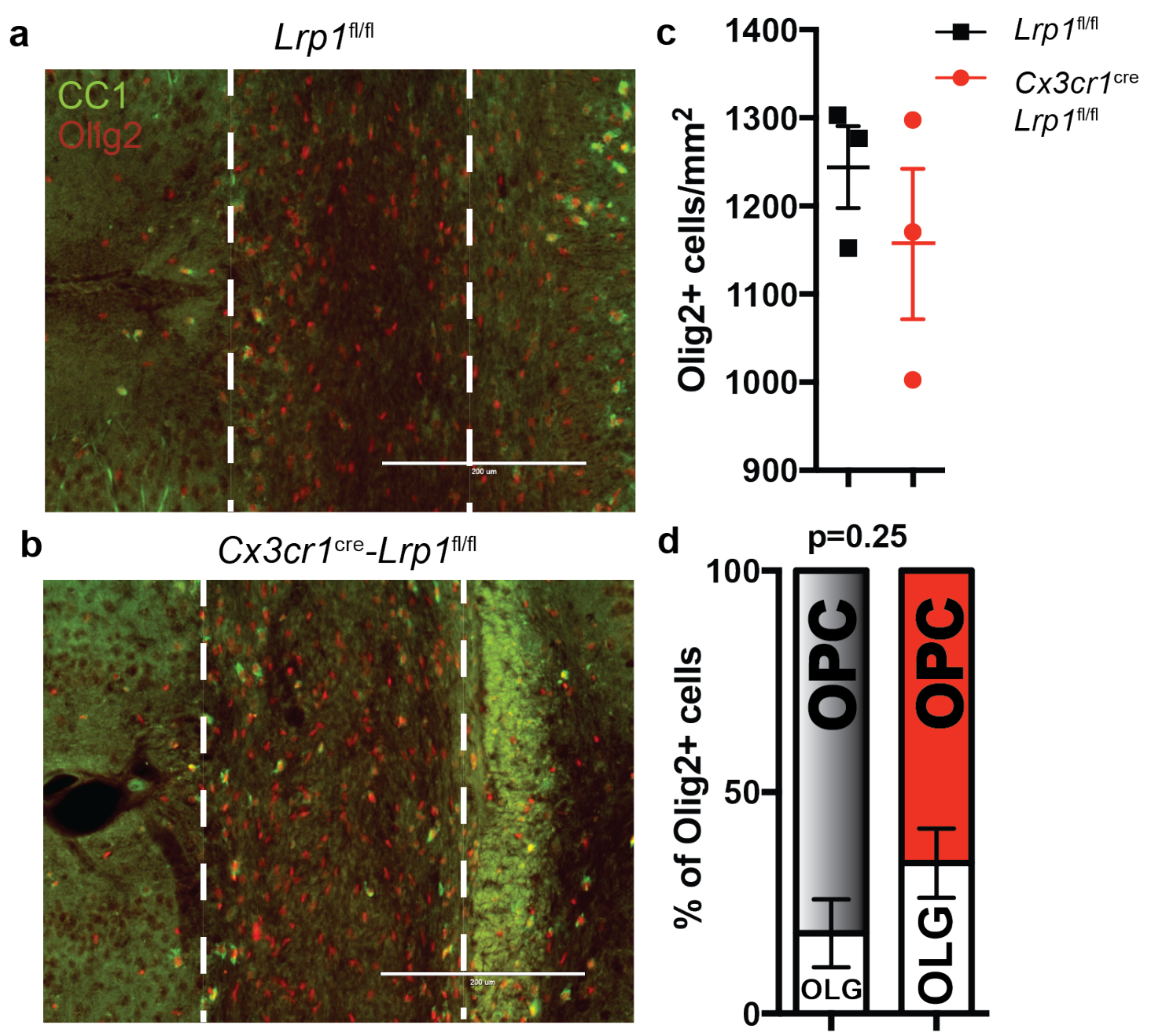

$C x 3 c r 1^{\text {cre }}-L r p 1^{\mathrm{fl} / \mathrm{fl}}$ and control $L r p 1^{\mathrm{fl} / \mathrm{fl}}$ mice were given chow containing $0.3 \%$ cuprizone for 5 weeks. The CNS was cryopreserved, sectioned, and stained with antibodies against $\mathrm{CC} 1$ (green) and Olig2 (red). Bars $=200 \mu \mathrm{M}$. Representative pictures of stained corpus callosum (white dotted line) from an a $C x 3 c r 1^{\mathrm{cre}}-\operatorname{Lrp} 1^{\mathrm{fl} / \mathrm{fl}}$ and $\mathbf{b}$ control $\left(\operatorname{Lrp} 1^{\mathrm{fl} / \mathrm{fl}}\right)$. c Number of cells of the oligodendrocyte lineage (Olig2+) normalized to area. d Percentage of mature oligodendrocytes $(\mathrm{CC} 1+/ \mathrm{Olig} 2+)$ and OPCs $(\mathrm{CC} 1+/ \mathrm{Olig} 2+-\mathrm{Olig} 2+$ cells). Student's t-Test; mean \pm s.e.m. 


\section{Chapter 5: SUMMARY}

In this work, I have extended the knowledge about the role of myeloid cells in MS. In particular, I have clarified the precise role of LRP1 in macrophages and microglia in a model of CNS autoimmunity. I also begin to address whether LRP1 deletion affects the phagocytic capability of myeloid cells, using in vitro models and a model of primary demyelination and remyelination in the CNS. This work has implications for LRP1's role as a molecular mediator between the pathways of inflammation and, potentially, phagocytosis.

Many groups have described the essential role that myeloid cells play in the pathogenesis of MS and the mouse model of MS, EAE. There are increased numbers of myeloid cells in the MS lesion, and the number of myeloid cells correlates directly with disease severity in MS ${ }^{21}$. These myeloid cells originate from the CNS resident microglia and the peripheral, monocyte-derived macrophages. Histologically, it is impossible to distinguish myeloid cells during neuroinflammation by unique protein markers, as even the recently discovered, microglia specific marker, purinergic receptor $\mathrm{P} 2 \mathrm{Y}$ (P2Y12) is known to be downregulated during microglia activation ${ }^{64,65}$.

One of the ways different groups have circumvented this problem is to use bone marrow transfer of GFP+ or RFP+ cells to study the role of peripheral vs CNS myeloid cells in $\mathrm{EAE}^{37,49}$. Although this method is useful in exploring the physiological roles of these different cell populations, it does not allow for specific targeting of gene expression in an unperturbed animal who has not been exposed to total body irradiation. Using a mouse strain that I generated, $C x 3 C r 1^{\text {creER }}-\operatorname{Lrp} 1^{\mathrm{fl} / \mathrm{fl}}$, I circumvented these issues to 
specifically delete our protein of interest, LRP1, in microglia. I also generated the strain $\mathrm{Cx} 3 \mathrm{Cr} 1^{\mathrm{cre}}-\mathrm{Lrp} 1^{\mathrm{fl} / \mathrm{fl}}$ to delete LRP1 in both microglia and peripheral-derived macrophages. As I used the $\mathrm{Cx} 3 \mathrm{Cr} I^{\text {creER }}-\operatorname{Lrp} 1^{\mathrm{fl} / \mathrm{fl}}$ mouse for the EAE disease model, I have shown that there were no differences in the number of microglia in the $C x 3 C r 1^{\text {creER }}-\operatorname{Lrp} 1^{\mathrm{fl} / \mathrm{fl}}$ mice compared to control. I ensured that LRP1 was deleted in the microglia, but not in the bone marrow derived macrophages, from the $C x 3 C r 1^{\text {creER }}-L_{r p} I^{\text {fl/fl }}$ mouse. I also show that the $\mathrm{Cx} 3 \mathrm{Cr} 1^{\text {cre }}-\mathrm{Lrp}^{\mathrm{fl} / \mathrm{fl}}$ mice had LRP1 deleted in both their microglia and bone marrow-derived macrophage populations, and that the $C x 3 C r 1^{\text {cre }}-\operatorname{Lrp} 1^{\mathrm{fl} / \mathrm{fl}}$ mice mediated a more effective deletion of LRP1 in the macrophages compared to $L y s M^{\mathrm{cre}}-L r p 1^{\mathrm{fl} / \mathrm{fl}}$.

Our group and others have shown that LRP1 is a potent anti-inflammatory protein in macrophages ${ }^{131,137,143}$. Since inflammation is a known contributor to disease severity in MS and EAE, I hypothesized that deleting LRP1 in both the peripheral bone marrow derived macrophage cells and the CNS microglia should result in increased disease severity in mice with EAE. Unexpectedly, I found that mice with LRP1 deleted in their peripheral myeloid cells do not have a difference in disease progression, whereas mice with LRP1 deleted in their microglia experience a more severe EAE progression as shown by average disease score and increased incidence. Even with an increased dose of mycobacterium to induce a more severe disease, there is no difference in $L y s M^{\text {cre }}-L r p 1^{\mathrm{fl} / \mathrm{fl}}$ mice and control. Hypotheses to explain this difference will be discussed in the future directions section, but they reflect our increasing appreciation of the dichotomous roles of these two populations during EAE. As a critical control, I showed that CX3CR1 haplodeficiency does not affect EAE progression in our mice. This had been a controversial point in the literature, as CX3CR1-deficient mice had been shown to both 
exhibit worse and better EAE outcome, while CX3CR1 haplodeficiency was suggested as a contributor to EAE severity ${ }^{177-179}$. In my hands, I have clearly shown that CX3CR1 haplodeficiency has no effect on disease outcome.

Higher disease score suggests that there is increased amount of demyelinating activity in the microglial LRP1 deficient mice. Thus, I stained the spinal cords with Luxol Fast Blue and found that indeed, the mice did have increased demyelination. This histological indication of disease severity correlated with an increased number of CD3+ T cells and Iba1+ myeloid cells in the spinal cord at disease onset. Using flow cytometry, I was able to distinguish between the infiltrating peripheral macrophages and the microglia. In the literature, a difference in disease severity is associated with an increased number of T cells and peripheral myeloid cells in the CNS, which is reflected in my flow cytometry data. However, published data from reporter mice also shows that there is a significant increase in the numbers of microglia when EAE is more severe, but this trend is not as clear in our $C x 3 C r 1^{\text {creER }}-L r p 1^{\mathrm{fl} / \mathrm{fl}}$ mice. The lack of significantly increased microglia numbers in the $C x 3 C r 1^{\text {creER }}-\operatorname{Lrp} 1^{\mathrm{fl} / \mathrm{fl}}$ mice, despite increased disease severity, demonstrates that it is possible LRP1 deficient microglia may be less proliferative in response to inflammatory stimulus. This would be interesting to explore in the context of other CNS inflammatory stimuli. Lastly, the lack of difference between MHCII expression in microglia and macrophages isolated from $C x 3 C r 1^{\mathrm{creER}}-\operatorname{Lrp} 1^{\mathrm{fl} / \mathrm{fl}}$ and $\operatorname{Lrp} 1^{\mathrm{fl} / \mathrm{fl}}$ mice suggests that activation is not affected. Another useful piece of information would be to examine whether there are other inflammatory and activation marker differences, such as CD80, CD86, TNF $\alpha$, and IL-1 $\beta$, on these myeloid cells during EAE. 
Since CX3CR1 is expressed on a subset of peripheral dendritic cells, and LRP1 has been implicated in the processing of antigen peptides ${ }^{33,164}$, I next sought to determine whether the antigen recall response in the periphery was perturbed in $C \times 3 C r I^{\text {creER }}-\operatorname{Lrp}^{\mathrm{fl} / \mathrm{fl}}$ mice. Antigen recall and $\mathrm{T}$ cell proliferation assays revealed that there was no difference between the two strains. These results indicate that there is no peripheral antigen presentation defect in the $C x 3 C r I^{\text {creER }}-\operatorname{Lrp}^{\mathrm{fl} / \mathrm{fl}}$ mice that could account for the difference in disease phenotype.

Microglial activation and LRP1 expression are both implicated in the maintenance of the blood brain barrier ${ }^{150,166,180}$. Autopsy histological analysis reveals perivascular cuffs of leukocytes in MS autopsy samples which are also thought to associate with regions of 'leaky' blood brain barrier ${ }^{181}$. Thus, we sought to assay the integrity of the blood brain barrier in $C x 3 C r 1^{\text {creER }}-\operatorname{Lrp} 1^{\mathrm{fl} / \mathrm{fl}}$ mice using a well-established model of blood brain barrier permeability ${ }^{167}$. There is an increase in blood-brain barrier permeability in both $C x 3 C r 1^{\text {creER }}-L r p I^{\mathrm{fl} / f \mathrm{l}}$ and control mice after LPS administration, but I found no difference in the amount of fluorescein between these groups, indicating that LRP1 deficiency in microglia does not impact the blood brain barrier.

Microglia morphology is a strong indicator of activation state. Microglia are normally ramified with branched, elongated processes. Inflammatory stimuli, such as systemic injection of LPS, lead to microglia activation ${ }^{167,168}$. When activated, microglia attain an amoeboid morphology where the processes are retracted and soma size is increased ${ }^{182}$. This morphological change with LPS administration is clearly shown in the control $\operatorname{Lrp} 1^{\mathrm{fl} / \mathrm{fl}}$ mouse. However, in the $C x 3 C r 1^{\text {creER }}-\operatorname{Lrp} I^{\mathrm{fl} / \mathrm{fl}}$ mouse, we can see a clear increase in the number of branched processes and an altered, activated morphology even 
without LPS injection. These results, along with the quantitation, clearly show that LRP1 deficient microglia are already activated at baseline. Further in situ analysis of cell activation in this model would be extremely beneficial in understanding how the microglia are activated. Assessing proliferation, expression of inflammatory cytokines, and lysosomal activity could all address possibilities for what may be activating the LRP1 deficient microglia at baseline. The microglial specific targeting of the $\mathrm{C} x 3 \mathrm{Cr} 1^{\mathrm{creER}}-\mathrm{Lrp}^{\mathrm{fl} / \mathrm{fl}}$ mouse did not impact the weights of mice, which were similar to the control animals. Since weight loss in sepsis models is a marker of systemic inflammation, this suggests that the peripheral response to LPS is unaltered in $C x 3 C r 1^{\text {creer }}-L_{r p} 1^{\mathrm{fl} / \mathrm{fl}}$ mice.

Since the morphology we observe in the LRP1 deficient microglia indicates an increased, pro-inflammatory state, we isolated microglia from $C x 3 C r I^{\text {cre }}-L_{r p} I^{\mathrm{fl} / \mathrm{ll}}$ mice and analyzed the production of inflammatory cytokine TNF $\alpha$ by qPCR and ELISA. It is well known that TNF $\alpha$ expression is correlated with disease severity in MS/EAE, and there have been multiple efforts to target this cytokine to assess whether disease outcomes can be improved ${ }^{81-83}$. TNF $\alpha$ expression is increased in LRP1 deficient microglia, and the increase is comparable in LRP1 deficient bone marrow-derived macrophages from the same mice. This corresponds to previous observations that LRP1 functions as an inhibitor of the inflammatory response. The production of inflammatory cytokines IL-6 and IL-1 $\beta$ in LRP1 deficient macrophages is also increased, and these same cytokines are increased during EAE in the CNS of $C x 3 C r 1^{\text {creER }}-\operatorname{Lrp} 1^{\mathrm{fl} / \mathrm{fl}}$ mice. Thus, it appears that microglia lacking LRP1 expression have an increased pro-inflammatory morphology and cytokine production, which may be responsible for the increased EAE disease severity in $C x 3 C r 1^{\text {creER }}-\operatorname{Lrpl}^{\mathrm{fl} / \mathrm{fl}}$ mice. In order to determine whether this pro- 
inflammatory signature was present in the LRP1 deficient microglia during EAE, we also isolated microglia during disease and analyzed activation markers by qPCR analysis. The LRP1 deficient microglia showed a trend towards increased inflammatory cytokine production (TNF $\alpha$, IL-6) and activation of the reactive oxygen species production pathway (NOS2, Arg). This pro-inflammatory signature was specifically present on LRP1 deficient microglia during EAE.

Production of the inflammatory cytokines TNF $\alpha$, IL-6 and IL-1 $\beta$, is meticulously regulated by the master transcriptional factor NF- $\mathrm{kB}$. Although constitutively expressed, $\mathrm{NF}-\kappa \mathrm{B}$ translocates to the nucleus only when its repressor I $\kappa \mathrm{B}$ undergoes phosphorylation by the regulatory IкB kinase (IKK) complex. Since work from our lab and others have shown that myeloid cells lacking LRP1 have an increased NF- $\mathrm{KB}$ activation signature, we sought to determine whether this could explain the increased inflammatory cytokine production in LRP1 deficient myeloid cells ${ }^{137}$. Protein immunoblot reveals that there is

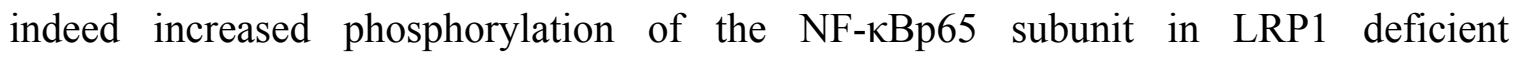
macrophages treated with LPS. To investigate potential cytoplasmic proteins involved in NF- $\kappa B$ activation that could be interacting with LRP1, we decided to use a panel of TLR ligands to test whether LRP1 showed specificity for the different adaptor protein pathways. TLR4, which is stimulated by LPS, is the only TLR that uses both the TRIF and MyD88 adaptor protein pathways. TLR3 only uses the TRIF dependent pathway, and all other TLRs use the MyD88 adaptor pathway. We sought to determine whether the expression of NF- $\kappa$ B regulated inflammatory cytokine, IL-6, changes in LRP1 deficient macrophages when treated with different TLR ligands. Our results indicate that LRP1 acts on the MyD88 dependent pathway to decrease the inflammatory NF- $\mathrm{KB}$ response, as 
the TRIF dependent pathway did not show an LRP1 dependent increase in IL-6 expression. We discuss potential binding partners and mediators of LRP1's antiinflammatory effect more thoroughly in the next section.

Besides regulating inflammation, LRP1 is also important in the phagocytic process. Based on the analysis of scavenger receptor expression and electron microscopy studies, it is now clear that microglia and peripherally derived macrophages participate in the phagocytosis of debris during $\mathrm{EAE}^{49,153}$. To probe in the contribution of LRP1 to phagocytosis during EAE, we looked at expression of lysosomal genes known to be upregulated during phagocytosis in flow sorted macrophages and microglia. Expression of the transcription factor TFEB, which is a master regulator of lysosome generation, is decreased in LRP1 deficient microglia. This suggests that there is a potential phagocytic defect in LRP1 deficient microglia, as TFEB expression was not altered in the macrophage population. Since these samples were taken from disease onset (Day 16), a more compelling difference may have been apparent during the chronic phase. Also, Mice that had EAE scores below 3 had poor microglial RNA quality, probably due to the lack of healthy cells after sorting. Therefore, it would be interesting to repeat the experiment at the chronic stage of disease to see if the lysosomal biogenesis genes are further altered. This data also presents an interesting link between the phagocytic capabilities and the inflammatory signature of the microglia. From our data, LRP1 deficient microglia seem more activated at baseline by morphology and are more proinflammatory with an LPS stimulus. Activation of myeloid cells usually leads to an increase in phagocytosis, but as LRP1 is a phagocytic receptor, the LRP1 deficient myeloid cell may also be deficient in the clearance process as well. 
In support of this hypothesis, LRP1 deficient microglia fail to engulf myelin, which could be contributing to the increased inflammatory environment during EAE in $C x 3 C r 1^{\text {creER }}-\operatorname{Lrp} 1^{\mathrm{fl} / \mathrm{fl}}$ mice. This failure to clear myelin debris would potentially result in an impaired remyelination. Surprisingly, LRP1 deficient macrophages have no different in the uptake of zymosan (TLR2) or E. coli (TLR4) ligand. These results suggest that LRP1 deficient myeloid cells do not exhibit an increased LPS response due to lack of engulfment of TLR2 and TLR4 ligands, which points to a downstream signaling effect of mediated by LRP1 in this context.

To see whether mice with LRP1 deficient myeloid cells would have problems with oligodendrocyte generation, we fed $C x 3 C r 1^{\text {cre }}-\operatorname{Lrp} 1^{\text {fl/fl }}$ mice with cuprizonesupplemented food to induce demyelination. We found that mice lacking LRP1 in their microglial population had significantly lower numbers of oligodendrocyte progenitor cells (OPC) in their white matter tract in the CNS. However, this method did not allow us to determine whether it is a problem of defective recruitment, proliferation, or increased apoptosis. Exploring these questions with analysis of the degradation of myelin in the corpus callosum would give us a better idea of how LRP1 expression on microglia may be affecting OPC development. Furthermore, behavioral testing and analysis of the white matter after the remyelination period would indicate whether the deletion of LRP1 on microglia is pathogenic in this mouse model. Since the mice used in this experiment have LRP1 deleted in both peripheral myeloid cells, whose extravasation into the brain during cuprizone induced demyelination remains controversial, and CNS microglia, we have also begun to explore the phenotype of $C x 3 C r 1^{\text {creER }}-\operatorname{Lrp}^{\mathrm{fl} / \mathrm{fl}}$ mice in the same model of demyelination/remyelination. The results will help determine whether peripheral-derived 
macrophages indeed enter the CNS during cuprizone treatment, and whether LRP1 deletion on microglia affects demyelination and remyelination. 


\section{Chapter 6: FUTURE DIRECTIONS}

In this work, I defined the participation of LRP1 on myeloid cells during the pathogenesis of disease in MS and EAE. I definitively showed that LRP1 expression is increased on myeloid cells during MS, and its expression on microglia is protective during EAE. LRP1 deficient microglia were found to be more pro-inflammatory and may also have deficient phagocytic ability, both of which would contribute to the exacerbation of disease. I will discuss potential future directions that arise from the results that I have obtained during my thesis.

\section{How does LRP1 inhibit NF-kB?}

LRP1 is widely known to be a scavenger receptor. However, our group and others have shown that LRP1 also regulates the activation of the inflammatory NF- $\mathrm{KB}$ signaling pathway ${ }^{134,137}$. In this work, I show for the first time that LRP1 can inhibit the MyD88 pathway downstream of the TLR signaling pathways. During MS, myeloid cells are a major producer of NF- $\mathrm{kB}$ regulated inflammatory cytokines such as TNF $\alpha$ and IL-6. Expression of these cytokines correlates to the severity of symptoms of MS. Therefore, exploiting the mechanism by which LRP1 mediates its anti-inflammatory effect is extremely desirable for future development of therapeutics.

Although my work describes MyD88 as an essential target of LRP1 mediated anti-inflammatory function, I was unable to identify the full signaling cascade involved in this new pathway. Using a fusion protein corresponding to the cytoplasmic domain of LRP1 as bait, I performed a mass spectrometry screen with protein lysates isolated from LPS treated primary murine macrophages. With this method, we identified two putative 
partners involved in the NF- $\mathrm{B}$ signaling pathway: TRAF6 (TNF receptor associated factor-6). TRAF6 acts as an activator, and A20 as an inhibitor, of the NF- $\kappa$ B response. Here, I discuss experiments undertaken to determine whether the expression of these proteins changes in response to LRP1 deletion, as well as attempts to specifically look at binding interaction of these proteins with LRP1. I will then go on to speculate how LRP1 could differentially modulate these proteins, and future potential experiments to further probe the interaction between TRAF6 or A20 and LRP1.

As a protein signal transducer specifically activated in the MyD88 adapter pathway and known to directly bind TAK1, a NF- $\mathrm{B}$ regulatory kinase, TRAF6 offered what seemed an exciting answer to our MyD88 specific mechanism ${ }^{183-186}$. Upon activation, TRAF6 undergoes Lys63-linked auto-ubiquitination and binds to the downstream signaling TAK1 protein complex ${ }^{185,}{ }^{187}$. TAK1 is activated and then mediates phosphorylation of the IKK complex to activate the NF- $\mathrm{BB}$, as well as JNK and p38 signaling pathways ${ }^{185}$. In addition to TAK1 binding, TRAF6 also mediates TNFR, IL-1R, and TLR family signaling through binding to a series of intermediates including serine/threonine kinases such as IRAKs ${ }^{188,}{ }^{189}$. TRAF6 expression is upregulated with activation of TLR signaling ${ }^{190}$, while its auto-ubiquitination is regulated by interaction with proteins such as another E3 ubiquitin ligase, cullin- $5^{191}$.

The literature provides multiple lines of evidence for TRAF6 regulation at the expression and post-translational modification level. Thus, I conducted experiments to address the hypothesis that TRAF6 expression is changed in LRP1 deficient cells and that TRAF6 specifically binds to LRP1. First, I did not detect changes in TRAF6 protein expression in LRP1 deficient bone marrow derived macrophages by immunoblot. 
Second, co-immunoprecipitation pull down with either LRP1 or TRAF6 antibody from primary murine macrophages after LPS treatment did not reveal any protein interaction. Third, I transfected plasmid expressing FLAG-TRAF6 ${ }^{192}$ into the murine macrophage cell line (RAW264.7) and precipitated either the overexpressed TRAF6, or LRP1, but again could not detect interaction between these two proteins. It is possible that the time points selected for analysis of the cellular lysates did not correlate with the temporal binding of LRP1 and TRAF6, as their interaction could be very transient, or that the amount of protein bound was too low to be detected. Another possibility is that the interaction between LRP1 and TRAF6 may be mediated by a post-translational modification, as TRAF6 activity is linked to ubiquitination.

These limitations could be addressed by using an imaging based protein interaction detection method, a proximity ligation assay (PLA). The advantages of this method include single molecule resolution, high specificity and sensitivity, as well as the ability to be applied to fixed MS tissue specimens. The PLA method depends on dual proximal binding by pairs of detection reagents, bound to antibodies against the proteins of interest, to generate amplifiable DNA strands ${ }^{193}$. This method can be further extended to allow detailed studies of protein phosphorylation events in situ. To explore the binding and/or phosphorylation of TRAF6 or A20 in relation to LRP1, I would culture bone marrow derived macrophages that are either LRP1 expressing or deficient. I would then treat them with LPS at different time points, fix the cells, and then incubate the cells with LRP1 and TRAF6 specific antibody raised in different species. After removal of the primary antibodies, I would then incubate the cells with species-specific secondary antibody each conjugated with a unique DNA strand. When the secondary antibodies are 
in close proximity, enzymatic ligation allows the DNA strands to form a circle. Polymerase activity amplifies the DNA product signal and the labeled detection probes then bind the product, which can be imaged as spots of high concentrations of fluorescence by microscopy ${ }^{193,194}$. This would allow for high-resolution detection of interaction between TRAF6 and LRP1.

In addition to the experiments looking at LRP1 binding to TRAF6, I also explored the potential interaction between LRP1 and A20. A20 was previously identified in multiple GWAS studies as a susceptibility gene for inflammatory disease and functions as a deubiquitinase involved in multiple pathways regulating NF- $\mathrm{\kappa B}$ activation, including TRAF6 ubiquitination ${ }^{195}$. A20 expression is rapidly induced upon NF-kB activation and mice deficient for A20 die prematurely due to severe multiorgan inflammation ${ }^{195,196}$. Due to its potent anti-inflammatory activity, A20 expression and activity are tightly regulated at the transcriptional level and by post-transcriptional modifications, such as phosphorylation ${ }^{197}$. Therefore, I measured A20 expression by qPCR from LRP1 expressing and deficient macrophages, but saw no difference in mRNA expression level. Other members in the laboratory also conducted co-immunopreciptation experiments with LRP1 and A20, but could not detect an interaction. For future investigation, I could further explore potential effects of LRP1 on translational and post-translational modifications of A20 by using the PLA method, described previously in this section, to detect phosphorylated forms of A20 and potential binding activity to LRP1.

I predict that the PLA method will reveal interaction between activated TRAF6 and/or A20 with LRP1 through the presence of foci with high fluorescence intensity with imaging, which will also localize the protein-protein interaction within the cell itself. 
However, the mechanistic interpretation would differ between the two proteins. As TRAF6 is a signal transducer, discovery of direct LRP1 binding could suggest that LRP1 disrupts TRAF6 ubiquitination or binding to its conserved recognition motif on upstream signaling proteins (Fig. 22). Alternatively, LRP1 could work as a sponge, titrating TRAF6 away from the TAK1 signaling complex and therefore turning off the signaling cascade. Conversely, A20 is an inhibitor of NF- $\mathrm{kB}$ activation and is regulated by both phosphorylation and expression. Therefore, if LRP1 is found to bind to A20, this could mean that LRP1 promotes A20 activity by enhancing its phosphorylation or promoting the deubiquitinase activity of A20 by enhancing binding to its target proteins for deubiquitination. Another option is that LRP1 could be necessary to recruit A20 at the TLR receptor-signaling complex at the plasma membrane to turn off the signaling pathway.

In sum, in this work I showed for the first time that downstream of TLR signaling, LRP1 specifically inhibits the MyD88 pathway in NF-kB activation. Our lab previously showed that LRP1 affects signaling downstream of other inflammatory signals, such as $\mathrm{TNF}^{134}$, and that the shed form of LRP1 also inhibits TNF $\alpha$ mediated NF-kB signaling ${ }^{136}$. Thus, it is possible that there is a common adaptor protein responsible for the anti-inflammatory effects downstream of TLR and TNFR signaling mediated by LRP1. Our laboratory had conducted a screen for LRP1 binding ligands and found potential candidates for its anti-inflammatory effect, including the TRAF6 adaptor protein and the NF-kB regulatory protein A20, both involved in the MyD88 pathway. However, I was unable to detect LRP1 mediated regulation of these putative proteins by co-immunoprecipitation or qPCR analysis. Further experiments, such as the PLA based 
method proposed or another candidate screen may be required to find the binding partner of LRP1 in the inflammatory pathway.

The NF-kB pathway is known to be highly active in myeloid cells in the MS lesion ${ }^{198}$. Within the CNS, NF- $\mathrm{KB}$ is constitutively active at high basal levels in neurons, but is only found in glial cells during stressed or pathological conditions ${ }^{199}$. In vivo administration of a peptide that disrupts $\mathrm{NF}-\mathrm{\kappa B}$ activation protects mice against $\mathrm{EAE}^{200}$. Thus, specifically targeting the NF- $\mathrm{KB}$ response during MS is highly desirable as a therapeutic target. Current first line inflammatory treatments which decrease NF- $\mathrm{BB}$ mediated signaling include glucocorticoids, but these drugs also have unwanted side effects stemming from transactivation of other signaling pathways ${ }^{201}$. Thus, drug companies have focused on developing inhibitors of individual NF-kB proteins, smallcompound molecules or ubiquitination inhibitors ${ }^{73}$. Proteins regulating NF- $\mathrm{\kappa B}$ activation, such as LRP1, would also be excellent targets for development of therapeutics. Upregulating the expression of LRP1 by increasing signaling through sterol regulatory element binding protein regulation or modulating activity of LRP1 binding to TRAF6 and A20 (Fig. 22) would provide potential avenues of therapeutic development with better specificity than glucocorticoid treatment during MS.

\section{Is the inflammatory phenotype of LRP1 deficient microglia exacerbated by a phagocytic deficit?}

Not only is LRP1 intricately involved in the modulation of inflammation, it is also involved in the phagocytic process by mediating the recognition and binding of up to 100 ligands $^{120}$. Our lab showed that LRP1 deficient cells are impaired in their myelin 
phagocytic capability, and it is thought that the amount of myelin debris may regulate inflammatory responses in MS patients as well as in cuprizone induced demyelination ${ }^{202}$.

Several studies suggest that during MS, phagocytosis and inflammation are cooperating in an intricate crosstalk. Monocytes derived from MS patients are deficient in phagocytosis of myelin ${ }^{203}$. Addition of a PPAR $\gamma$ (peroxisome proliferator-activated receptor $\gamma$ ) agonist, an inflammation-modulating drug clinically used to treat diabetes, decreases the inflammatory profile in these MS patient-derived monocytes and correlates to an increase in their myelin phagocytic capability ${ }^{203}$. Further, while there are a multitude of papers showing that microglial activation and their subsequent inflammatory response are implicated in the initiation of $\mathrm{EAE}^{63,159,204}$, microglial phagocytosis is also altered during neuroinflammatory conditions such as stroke and Alzheimer's ${ }^{205}$.

In this work, I showed that deletion of LRP1 causes an increased NF- $\kappa$ B mediated inflammation, which could contribute to disease pathogenesis in EAE. Since LRP1 is a phagocytic receptor, it is possible that the LRP1 deficient microglia also have defected phagocytosis, and this could explain the phenotypic difference between EAE progression in mice with either LRP1 deficient microglia or peripherally derived macrophages. Microglia are thought to be the major phagocytic myeloid cell population during early onset of disease in $\mathrm{EAE}^{206}$. Literature suggests that microglial phagocytic activity may be beneficial, whereas monocyte-derived macrophage phagocytic activity may be detrimental, during $\mathrm{EAE}^{49}$. From these premises, I hypothesize that monocytes may be already primed to have maximal inflammatory activity when entering the CNS, so that deletion of LRP1 in this compartment does not cause further inflammation, whereas microglial phagocytic deficiency may contribute to disease pathogenesis due to increased 
inflammation in conjunction with lack of myelin clearance. These hypotheses require a detailed mechanistic understanding of the pre-clinical disease inflammatory priming and phagocytic capabilities of the myeloid cells involved in EAE.

An initial way to test these hypotheses would be to purify LRP1 expressing and deficient microglia from the adult CNS, treat them with myelin and TNF $\alpha / \mathrm{LPS}$ to determine whether inflammatory responses could increase or decrease myelin uptake. In detail, I could treat LRP1 expressing and deficient microglia with LPS or TNF $\alpha$, and then measure the phagocytic uptake of fluorescence myelin in conjunction with phosphorylated NF-kB by flow cytometry. Simultaneously, I could isolate LRP1 expressing microglia, treat them with LPS or TNF $\alpha$, fluorescent myelin and a LRP1 specific inhibitor, RAP, to determine whether NF- $\mathrm{KB}$ activation is changed by LRP1 inhibition. I could do this by conducting flow cytometry for phosphorylated NF- $\mathrm{kB}$ and qPCR for NF- $\kappa B$ regulated cytokine such as TNF $\alpha$ and IL-1 $\beta$. Since myelin phagocytosis is reported to mediate an anti-inflammatory effect on myeloid cells, I predict the results will indicate that lack of LRP1 expression cells or inhibition of LRP1 with RAP would ablate the anti-inflammatory effect of myelin phagocytosis.

There are a few limitations to this experiment, including possible astrocyte contamination of the culture and low yield of cells. In the literature, microglia isolated from neonatal mice are highly recommended for obtaining enough cells to perform complex experiments, but it has been shown that their transcriptome and behavior are quite different from adult microglia, and are more similar to the peripherally derived macrophages ${ }^{207-209}$. Therefore, these limitations must be taken into account when assessing in vitro functions of primary microglia. A few alternative approaches are 
possible, including using LRP1 deficient primary bone marrow macrophages or focusing on in vivo assays involving either administration of debris to the CNS, or inducing demyelination, as with the cuprizone model, in mice with LRP1 deficient microglia.

It is well established that lack of myelin debris clearance in MS is detrimental for a few reasons. First, myelin debris contains inhibitors of oligodendrocyte differentiation ${ }^{94}$. Increasing the amount of myelin debris in demyelinating lesions by injecting extrinsic myelin reduces the number of oligodendrocyte precursor cell differentiation ${ }^{94}$. Mechanistically, the RhoA-ROCK and PKC signaling pathways, activated by the presence of myelin debris, have been implicated as the inhibitors of OPC differentiation ${ }^{210}$. Second, presence of myelin debris increases microgliosis and the inflammatory response in mice, while samples of human MS lesions in the white matter had increased MHC II expression compared to gray matter ${ }^{202}$. Third, myelin debris may also contribute to the failure of axonal regeneration in the CNS after acute injury ${ }^{93}$. Collectively, the combination of these detrimental consequences suggests that myelin debris clearance is an excellent target for therapy. Indeed, it has been suggested that an optimal treatment strategy for MS could include boosting microglial phagocytic processes while limiting inflammatory responses ${ }^{211}$. As LRP1 functions as a phagocytic receptor for myelin and is also involved in the regulation of inflammatory responses, designing therapeutics to promote LRP1 function in both pathways would be an efficient double-edged sword in the treatment of MS. 


\section{Are there lysosomal changes related to phagocytic defects in LRP1 deficient microglia?}

Phagocytosis necessitates lysosomal function for digestion of accumulated debris. Lysosomes, long regarded as a simple acidic waste disposal of the cell, are now increasingly considered as a therapeutic target in diseases, including lysosomal storage disorders, cancer, and neurodegenerative disease ${ }^{212}$. Global disturbances in lysosome function have more severe consequences in the CNS, suggested to be due to the selective vulnerability of neurons ${ }^{212}$. However, it was shown that phagocytosis in antigen presenting cells also results in remodeling and relocalization of the lysosome ${ }^{213}$. Dysregulation of lysosomal function in microglia results in impairment of microglial development and accumulation of neuronal debris ${ }^{214}$. Within the context of MS, staining MS lesions for myelin lipids by Oil Red O (ORO) reveals that ORO+ macrophages are present even before apparent myelin loss in early lesions ${ }^{215}$. ORO + macrophages containing MBP peptide are found in the hypercellular, demyelinated MS lesion ${ }^{215}$. In addition to phagocytosing myelin, microglia are also found to phagocytose neuronal debris during MS. Axonal damage is associated with HLA-DR+ myeloid cells engulfing axonal bulbs and containing neuronal proteins ${ }^{216}$.

Therefore, identifying lysosomal changes would provide insight into disease related phagocytic processes during MS. A transcriptional network responsible for lysosomal biogenesis, regulated by the master transcription factor TFEB, was described within the last ten years ${ }^{92}$. TFEB is regulated at both the transcriptional and posttranslational level via phosphorylation ${ }^{92}$. I designed qPCR probes specific for TFEB as well as a series of genes downstream of TFEB regulation. I then isolated microglia and 
macrophages from microglial LRP1 expressing and deficient mice during EAE to identify if there are any changes in expression of TFEB, or downstream genes regulated by TFEB. The preliminary results indicate that it is possible the expression of genes involved in lysosome function is altered in LRP1 deficient microglia during EAE. However, a limitation of this finding is that the change is not significant, requiring an increased number of samples for analysis. Furthermore, the results do not include information on the expression of these genes at baseline before EAE onset during homeostasis.

It is also possible that LRP1 itself is somehow involved in lysosome generation, as in my results only a subset of genes known to be upregulated during lysosomal biogenesis showed a trend toward altered mRNA expression in LRP1 deficient microglia. To further test this hypothesis, I would first include an increased number of mice in my analysis. I would also use FACS to isolate LRP1 expressing and deficient microglia during EAE and plate them on cover slips to monitor lysosomal activity by assessing lysosomal $\mathrm{pH}$ and structure by using tools such as LysoTracker. I would also isolate spinal cords from $C x 3 c r 1^{\text {creER }}-L r p I^{\mathrm{fl} / \mathrm{fl}}$ mice during EAE and immunohistochemically stain the spinal cords for myeloid marker Iba-1, LRP1, lysosomal markers CD68, Lamp1, as well as myelin markers MBP and degraded MBP. This would allow for monitoring of lysosomal content of LRP1 expressing and deficient myeloid cells during EAE. A limitation of the immunohistochemical method is that I would not be able to differentiate between the infiltrating macrophages and resident microglia. However, a decrease in intracellular myelin and decreased lysosomal marker staining in mice with myeloid cells deficient in LRP1 would suggest that the phagocytic defect in LRP1 deficient microglia 
leads to altered lysosomal gene function. Further investigation into how LRP1 participates in the intracellular phagocytic machinery would be informative from the perspective of both cell biology and pathology.

Broadly speaking, the ability of the CNS to clear debris has been an area of ongoing investigative interest. In the PNS, Schwann cells assist the blood-derived macrophages to effectively clear myelin debris during injury and development ${ }^{217,218}$. However, myelin debris can linger for weeks to years in the CNS after an initial contusion in the cortical spinal tract ${ }^{219,220}$. It is thought that the suboptimal phagocytic activity of microglia contributes to this lack of clearance, leading to inhibition of axon regeneration ${ }^{221}$. Since functional lysosomes are essential for the proper progression of phagocytosis and autophagy, promoting lysosomal biogenesis through targeting the TFEB transcription pathway may provide an avenue for improving phagocytic clearance of debris in the CNS during MS. Phosphorylated TFEB is retained in the cytoplasm, whereas dephosphorylation causes translocation of TFEB into the nucleus to induce transcription of target genes ${ }^{222}$. Therefore a specific phosphatase for TFEB could be a viable therapeutic for increasing lysosomal digestion of phagocytosed debris. TFEB is also known to be phosphorylated by mTORC1 and ERK2 kinase, so methods to enhance mTORC1 and ERK2 activity would also be an option for increasing lysosomal function $^{222}$. TFEB overexpression or pharmacological activation in cellular and mouse models of Alzheimer's disease has also been shown to reduce the amount of protein aggregates in the $\mathrm{CNS}^{223-225}$. Using a similar method may prove extremely fruitful in MS, potentially decreasing the amount of myelin debris in the CNS, thereby promoting axonal regrowth and remyelination. 


\section{How does microglial LRP1 deficiency affect remyelination?}

Phagocytosis of myelin is known to be critical to the remyelinating process, as myelin debris lingering in the lesions have been shown to inhibit myelin repair ${ }^{95}$. Comparison of myelin isolated from normal appearing white matter in control vs. MS donor brains revealed that myelin from MS brains was phagocytosed more efficiently by a human macrophage cell line and primary human microglia in vitro ${ }^{226}$. Even if much more work is needed to understand why debris are not cleared properly in the CNS, this data suggest that it is probably due to a combination of cell intrinsic and extrinsic factors present in the lesion environment. Therefore, modulating the phagocytic ability of myeloid cells during MS may be a potential target mechanism for further therapeutic investigation. For example, glatiramer acetate, a proven MS therapeutic, was shown to increase phagocytic activity of human monocytes in MS patients, demonstrating the beneficial effect of promoting waste clearance ${ }^{227}$.

LRP1, as discussed earlier, is a phagocytic receptor that recognizes myelin ${ }^{131}$. Since myeloid cell phagocytic ability is important in the clearance of myelin debris, utilizing an in vivo model of demyelination/remyelination would be informative as to the effects of LRP1 deletion on microglia on phagocytosis, and by proxy, remyelination. The timeline and phenotype of demyelination and remyelination is well described in the cuprizone chow model. Thus, I treated $C x 3 c r 1^{\text {cre }}-\operatorname{Lrp} 1^{\mathrm{fl} / \mathrm{fl}}$ mice with cuprizone and determined the numbers of oligodendrocytes and oligodendrocyte lineage cells during the demyelinating phase at 5 weeks of cuprizone treatment. My preliminary results suggest that the numbers of OPCs is decreased during the demyelinating phase of disease. For future investigation, the experiment needs to be repeated with an increased number of 
mice. Furthermore, immunofluorescence staining and quantification of the ratio between degraded MBP and MBP would be required to determine whether this decrease in OPCs is potentially due to lack of myelin debris clearance. The experiment also needs to be extended to the remyelination process, where the corpus callosum is analyzed after removing cuprizone from chow for two weeks, to determine if the extent of remyelination is altered in the myeloid LRP1 deficient mice.

If the preliminary observation that OPC number during demyelination is decreased holds true, I predict that myeloid LRP1 deficient mice will have increased dMBP staining during the demyelination and the remyelination period and loss of intracellular MBP stain in the myeloid cells. Following that, after remyelination these mice would have decreased number of oligodendrocytes and decreased MBP staining in the corpus callosum. Behavioral tasks reflecting this remyelination deficit could be used to assess phenotypic changes in myeloid LRP1 deficient mice, such as the Morris water maze.

Available therapeutics for MS largely target the infiltration of immune cells into, and their inflammatory activity within, the CNS. Although these disease-modifying drugs are efficacious in the prevention of relapses, they are unable to assist in the repair process in the brain to promote clearance of debris and promote remyelination. This long-term damage is thought to be the cause of increasing disability in MS patients. Therefore, there is great interest in elucidating the molecular mechanisms of remyelination. One of the crucial steps towards remyelination is the clearance of existing myelin debris. As LRP1 functions as a receptor for the phagocytosis of myelin and apoptotic cells, enhancing its function is potentially beneficial towards the clearance of debris and promotion of 
remyelination. Thus, targeting the phagocytic function of LRP1 would offer neuroprotective benefits from the viewpoint of CNS repair and restoration in MS.

In sum, these experiments would provide further insights into the function of LRP1 in microglia. Understanding how LRP modulates on myeloid cell activity in the CNS during homeostasis and pathology provides valuable pathophysiological context for the crosstalk between the processes of phagocytosis and inflammation. Therapeutically, biologics or small molecules that can enhance LRP1's phagocytic activity or antiinflammatory effect can potentially be developed for neuroinflammatory and demyelinating diseases. 
Figure 22. Schematic of the proposed interaction between LRP1 and TRAF6/A20

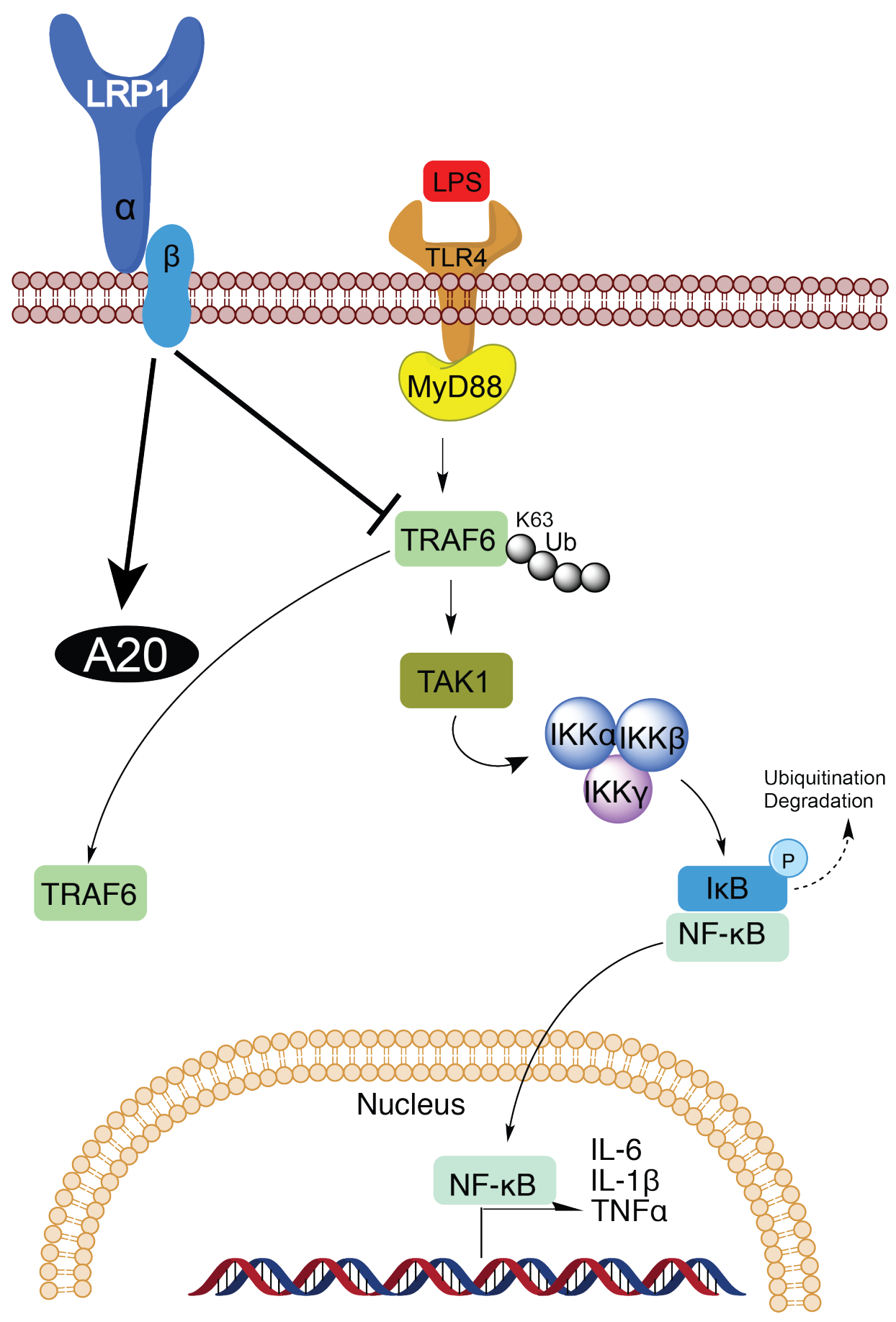




\section{Bibliography}

1. Chin JH, Vora N. The global burden of neurologic diseases. Neurology. 2014;83(4):349-351. doi: 10.1212/WNL.0000000000000610.

2. Adelman G, Rane SG, Villa KF. The cost burden of multiple sclerosis in the United States: a systematic review of the literature. J Med Econ. 2013;16(5):639-647. doi: 10.3111/13696998.2013.778268 [doi].

3. Souza A, Kelleher A, Cooper R, Cooper RA, Iezzoni LI, Collins DM. Multiple sclerosis and mobility-related assistive technology: systematic review of literature. $J$ Rehabil Res Dev. 2010;47(3):213-223.

4. Kumar DR, Aslinia F, Yale SH, Mazza JJ. Jean-Martin Charcot: The Father of Neurology. Clinical Medicine \& Research. 2009;9(1):46-49. doi: 10.3121/cmr.2009.883.

5. Steinman L. Immunology of relapse and remission in multiple sclerosis. Annu Rev Immunol. 2014;32:257-281 doi: 10.1146/annurev-immunol-032713-120227 [doi].

6. Compston A, Coles A. Multiple sclerosis. Lancet. 2008;372(9648):1502-1517. doi: 10.1016/S0140-6736(08)61620-7; 10.1016/S0140-6736(08)61620-7.

7. Sospedra M, Martin R. IMMUNOLOGY OF MULTIPLE SCLEROSIS. Annu Rev Immunol. 2005;23(1):683-747. doi: 10.1146/annurev.immunol.23.021704.115707.

8. Hammond SR, English DR, McLeod JG. The age-range of risk of developing multiple sclerosis. Brain. 2000;123(5):968-974. doi: 10.1093/brain/123.5.968. 
9. Bashinskaya VV, Kulakova OG, Boyko AN, Favorov AV, Favorova OO. A review of genome-wide association studies for multiple sclerosis: classical and hypothesis-driven approaches. Hum Genet. 2015;134(11):1143-1162. doi: 10.1007/s00439-015-1601-2.

10. Stys PK. Pathoetiology of multiple sclerosis: are we barking up the wrong tree?. F1000Prime Rep. 2013;5:20-20. Print 2013. doi: 10.12703/P5-20 [doi].

11. Stys PK, Zamponi GW, van Minnen J, Geurts JJ. Will the real multiple sclerosis please stand up?. Nat Rev Neurosci. 2012;13(7):507-514. doi: 10.1038/nrn3275 [doi].

12. Traka M, Podojil JR, McCarthy DP, Miller SD, Popko B. Oligodendrocyte death results in immune-mediated CNS demyelination. Nat Neurosci. 2016;19(1):65-74.

13. Prineas J. Pathology of the early lesion in multiple sclerosis. Hum Pathol. 1975;6(5):531-554.

14. Gold SM, Mohr DC, Huitinga I, Flachenecker P, Sternberg EM, Heesen C. The role of stress-response systems for the pathogenesis and progression of MS. Trends Immunol. 2005;26(12):644-652. doi: S1471-4906(05)00244-9 [pii].

15. Friese MA, Fugger L. T cells and microglia as drivers of multiple sclerosis pathology. Brain. 2007;130(11):2755-2757. doi: 10.1093/brain/awm246.

16. Lucchinetti C, Bruck W, Parisi J, Scheithauer B, Rodriguez M, Lassmann H. Heterogeneity of multiple sclerosis lesions: implications for the pathogenesis of demyelination. Ann Neurol. 2000;47(6):707-717.

17. Lucchinetti CF, Bruck W, Lassmann H. Evidence for pathogenic heterogeneity in multiple sclerosis. Ann Neurol. 2004;56(2):308. doi: 10.1002/ana.20182. 
18. Wilkins A, Majed H, Layfield R, Compston A, Chandran S. Oligodendrocytes promote neuronal survival and axonal length by distinct intracellular mechanisms: a novel role for oligodendrocyte-derived glial cell line-derived neurotrophic factor. $J$ Neurosci. 2003;23(12):4967-4974. doi: 23/12/4967 [pii].

19. Bjartmar C, Wujek JR, Trapp BD. Axonal loss in the pathology of MS: consequences for understanding the progressive phase of the disease. J Neurol Sci. 2003;206(2):165-171. doi: S0022510X02000692 [pii].

20. Polman CH, Reingold SC, Banwell B, et al. Diagnostic criteria for multiple sclerosis: 2010 Revisions to the McDonald criteria. Ann Neurol. 2010;69(2):292-302. doi: 10.1002/ana.22366

21. Vogel DY, Vereyken EJ, Glim JE, et al. Macrophages in inflammatory multiple sclerosis lesions have an intermediate activation status. J Neuroinflammation. 2013;10:35-209410-35. doi: 10.1186/1742-2094-10-35; 10.1186/1742-2094-10-35.

22. Bitsch A, Schuchardt J, Bunkowski S, Kuhlmann T, Bruck W. Acute axonal injury in multiple sclerosis. Correlation with demyelination and inflammation. Brain. 2000;123 ( Pt 6)(Pt 6):1174-1183.

23. Brochet B, Deloire MS, Touil T, et al. Early macrophage MRI of inflammatory lesions predicts lesion severity and disease development in relapsing EAE. Neuroimage. 2006;32(1):266-274. doi: 10.1016/j.neuroimage.2006.03.028.

24. Constantinescu CS, Farooqi N, O'Brien K, Gran B. Experimental autoimmune encephalomyelitis (EAE) as a model for multiple sclerosis (MS). $\mathrm{Br} J$ Pharmacol. 2011;164(4):1079-1106. doi: 10.1111/j.1476-5381.2011.01302.x. 
25. Praet J, Guglielmetti C, Berneman Z, Van der Linden A, Ponsaerts P. Cellular and molecular neuropathology of the cuprizone mouse model: Clinical relevance for multiple sclerosis. Neuroscience \& Biobehavioral Reviews. 2014;47:485-505. doi: http://dx.doi.org/10.1016/j.neubiorev.2014.10.004.

26. Gudi V, Gingele S, Skripuletz T, Stangel M. Glial response during cuprizone-induced de- and remyelination in the CNS: lessons learned. Frontiers in Cellular Neuroscience. 2014;8.

27. Bakker DA, Ludwin SK. Blood-brain barrier permeability during Cuprizone-induced demyelination: Implications for the pathogenesis of immune-mediated demyelinating diseases. $J$ Neurol Sci. 1987;78(2):125-137. doi: http://dx.doi.org/10.1016/0022-510X(87)90055-4.

28. Skripuletz T, Gudi V, Hackstette D, Stangel M. De- and remyelination in the CNS white and grey matter induced by cuprizone: the old, the new, and the unexpected. Histol Histopathol. 2011;26(12):1585-1597.

29. Goldberg J, Daniel M, van Heuvel Y, et al. Short-Term Cuprizone Feeding Induces Selective Amino Acid Deprivation with Concomitant Activation of an Integrated Stress Response in Oligodendrocytes. Cell Mol Neurobiol. 2013;33(8):1087-1098. doi: 10.1007/s10571-013-9975y.

30. Matsushima GK, Morell P. The Neurotoxicant, Cuprizone, as a Model to Study Demyelination and Remyelination in the Central Nervous System. Brain Pathology. 2001;11(1):107-116. doi: 10.1111/j.1750-3639.2001.tb00385.x.

31. McMahon EJ, Suzuki K, Matsushima GK. Peripheral macrophage recruitment in cuprizone-induced CNS demyelination despite an intact blood-brain barrier. J Neuroimmunol. 2002;130(1-2):32-45. doi: http://dx.doi.org/10.1016/S0165-5728(02)00205-9. 
32. Cho IH, Hong J, Suh EC, et al. Role of microglial IKKbeta in kainic acid-induced hippocampal neuronal cell death. Brain. 2008;131(Pt 11):3019-3033. doi: 10.1093/brain/awn230 [doi].

33. Jung S, Aliberti J, Graemmel P, et al. Analysis of fractalkine receptor CX(3)CR1 function by targeted deletion and green fluorescent protein reporter gene insertion. Mol Cell Biol. 2000;20(11):4106-4114.

34. Bauer J, Huitinga I, Zhao W, Lassmann H, Hickey WF, Dijkstra CD. The role of macrophages, perivascular cells, and microglial cells in the pathogenesis of experimental autoimmune encephalomyelitis. Glia. 1995;15(4):437-446. doi: 10.1002/glia.440150407.

35. Brosnan CF, Bornstein MB, Bloom BR. The effects of macrophage depletion on the clinical and pathologic expression of experimental allergic encephalomyelitis. J Immunol. 1981;126(2):614-620.

36. Huitinga I, van Rooijen N, de Groot CJ, Uitdehaag BM, Dijkstra CD. Suppression of experimental allergic encephalomyelitis in Lewis rats after elimination of macrophages. $J$ Exp Med. 1990;172(4):1025-1033.

37. Ajami B, Bennett JL, Krieger C, McNagny KM, Rossi FM. Infiltrating monocytes trigger EAE progression, but do not contribute to the resident microglia pool. Nat Neurosci. 2011;14(9):1142-1149. doi: 10.1038/nn.2887; 10.1038/nn.2887.

38. Fife BT, Huffnagle GB, Kuziel WA, Karpus WJ. CC chemokine receptor 2 is critical for induction of experimental autoimmune encephalomyelitis. J Exp Med. 2000;192(6):899-905. 
39. Hume DA, MacDonald KPA. Therapeutic applications of macrophage colonystimulating factor-1 (CSF-1) and antagonists of CSF-1 receptor (CSF-1R) signaling. Blood. 2011;119(8):1810-1820.

40. Auffray C, Fogg D, Garfa M, et al. Monitoring of blood vessels and tissues by a population of monocytes with patrolling behavior. Science. 2007;317(5838):666-670. doi: 317/5838/666 [pii].

41. Auffray C, Sieweke MH, Geissmann F. Blood monocytes: development, heterogeneity, and relationship with dendritic cells. Annu Rev Immunol. 2009;27:669-692. doi: 10.1146/annurev.immunol.021908.132557 [doi].

42. Geissmann F, Auffray C, Palframan R, et al. Blood monocytes: distinct subsets, how they relate to dendritic cells, and their possible roles in the regulation of T-cell responses. Immunol Cell Biol. 2008;86(5):398-408.

43. Takeuchi O, Akira S. Pattern Recognition Receptors and Inflammation. Cell. 2010;140(6):805-820. doi: http://dx.doi.org/10.1016/j.cell.2010.01.022.

44. Izikson L, Klein RS, Charo IF, Weiner HL, Luster AD. Resistance to experimental autoimmune encephalomyelitis in mice lacking the CC chemokine receptor (CCR)2. J Exp Med. 2000;192(7):1075-1080.

45. Boring L, Gosling J, Chensue SW, et al. Impaired monocyte migration and reduced type 1 (Th1) cytokine responses in $\mathrm{C}-\mathrm{C}$ chemokine receptor 2 knockout mice. J Clin Invest. 1997;100(10):2552-2561. doi: 10.1172/JCI119798 [doi].

46. Kurihara T, Warr G, Loy J, Bravo R. Defects in macrophage recruitment and host defense in mice lacking the CCR2 chemokine receptor. $J$ Exp Med. 1997;186(10):1757-1762. 
47. Kruglov AA, Lampropoulou V, Fillatreau S, Nedospasov SA. Pathogenic and Protective Functions of TNF in Neuroinflammation Are Defined by Its Expression in $\mathrm{T}$ Lymphocytes and Myeloid Cells. The Journal of Immunology. 2011;187(11):5660-5670. doi: 10.4049/jimmunol.1100663.

48. Lévesque SA, Paré A, Mailhot B, et al. Myeloid cell transmigration across the CNS vasculature triggers IL-1 $\beta$ driven neuroinflammation during autoimmune encephalomyelitis in mice. The Journal of Experimental Medicine. 2016. doi: 10.1084/jem.20151437.

49. Yamasaki R, Lu H, Butovsky O, et al. Differential roles of microglia and monocytes in the inflamed central nervous system. The Journal of Experimental Medicine. 2014;211(8):1533-1549. doi: 10.1084/jem.20132477.

50. Ellrichmann G, ThÃIne J, Lee D, Rupec RA, Gold R, Linker RA. Constitutive activity of NF-kappa B in myeloid cells drives pathogenicity of monocytes and macrophages during autoimmune neuroinflammation. Journal of Neuroinflammation. 2012;9(1):1-11. doi: 10.1186/1742-2094-9-15.

51. Hao W, Decker Y, Schnoder L, et al. Deficiency of IkappaB Kinase beta in Myeloid Cells Reduces Severity of Experimental Autoimmune Encephalomyelitis. Am J Pathol. 2016. doi: S0002-9440(16)00083-3 [pii].

52. Bellingan GJ, Caldwell H, Howie SE, Dransfield I, Haslett C. In vivo fate of the inflammatory macrophage during the resolution of inflammation: inflammatory macrophages do not die locally, but emigrate to the draining lymph nodes. J Immunol. 1996;157(6):2577-2585. 
53. Ginhoux F, Greter M, Leboeuf M, et al. Fate Mapping Analysis Reveals That Adult Microglia Derive from Primitive Macrophages. Science. 2010;330(6005):841-845. doi: 10.1126/science.1194637.

54. Ajami B, Bennett JL, Krieger C, Tetzlaff W, Rossi FM. Local self-renewal can sustain CNS microglia maintenance and function throughout adult life. Nat Neurosci. 2007;10(12):1538-1543. doi: nn2014 [pii].

55. Yona S, Kim KW, Wolf $\mathrm{Y}$, et al. Fate mapping reveals origins and dynamics of monocytes and tissue macrophages under homeostasis. Immunity. 2013;38(1):79-91. doi: 10.1016/j.immuni.2012.12.001 [doi].

56. Henson PM, Hume DA. Apoptotic cell removal in development and tissue homeostasis. Trends Immunol. 2006;27(5):244-250. doi: S1471-4906(06)00092-5 [pii].

57. Schafer DP, Lehrman EK, Kautzman AG, et al. Microglia sculpt postnatal neural circuits in an activity and complement-dependent manner. Neuron. 2012;74(4):691-705. doi: 10.1016/j.neuron.2012.03.026 [doi].

58. Paolicelli RC, Bolasco G, Pagani F, et al. Synaptic pruning by microglia is necessary for normal brain development. Science. 2011;333(6048):1456-1458. doi: 10.1126/science.1202529 [doi].

59. Sierra A, Encinas JM, Deudero JJ, et al. Microglia shape adult hippocampal neurogenesis through apoptosis-coupled phagocytosis. Cell Stem Cell. 2010;7(4):483-495. doi: 10.1016/j.stem.2010.08.014 [doi]. 
60. Nimmerjahn A, Kirchhoff F, Helmchen F. Resting microglial cells are highly dynamic surveillants of brain parenchyma in vivo. Science. 2005;308(5726):1314-1318. doi: 1110647 [pii].

61. Davalos D, Grutzendler J, Yang G, et al. ATP mediates rapid microglial response to local brain injury in vivo. Nat Neurosci. 2005;8:752-758.

62. Ponomarev ED, Shriver LP, Maresz K, Dittel BN. Microglial cell activation and proliferation precedes the onset of CNS autoimmunity. J Neurosci Res. 2005;81(3):374-389. doi: 10.1002/jnr.20488 [doi].

63. Heppner FL, Greter M, Marino D, et al. Experimental autoimmune encephalomyelitis repressed by microglial paralysis. Nat Med. 2005;11(2):146-152. doi: nm1177 [pii].

64. Haynes SE, Hollopeter G, Yang G, et al. The P2Y12 receptor regulates microglial activation by extracellular nucleotides. Nat Neurosci. 2006;9(12):1512-1519. doi: nn1805 [pii].

65. Butovsky O, Jedrychowski MP, Moore CS, et al. Identification of a Unique TGF$\beta$ Dependent Molecular and Functional Signature in Microglia. Nat Neurosci. 2013;17(1):131143. doi: 10.1038/nn.3599.

66. Martinez FO, Gordon S. The M1 and M2 paradigm of macrophage activation: time for reassessment. F1000Prime Reports. 2014;6:13. doi: 10.12703/P6-13.

67. Kawai T, Akira S. Signaling to NF-xB by Toll-like receptors. Trends Mol Med. 2007;13(11):460-469. doi: http://dx.doi.org/10.1016/j.molmed.2007.09.002. 
68. Hiscott J, Marois J, Garoufalis J, et al. Characterization of a functional NF-kappa B site in the human interleukin 1 beta promoter: evidence for a positive autoregulatory loop. Mol Cell Biol. 1993;13(10):6231-6240.

69. Shakhov AN, Collart MA, Vassalli P, Nedospasov SA, Jongeneel CV. Kappa B-type enhancers are involved in lipopolysaccharide-mediated transcriptional activation of the tumor necrosis factor alpha gene in primary macrophages. J Exp Med. 1990;171(1):35-47.

70. Fenner BJ, Scannell M, Prehn JHM. Expanding the Substantial Interactome of NEMO Using Protein Microarrays. PLoS ONE. 2010;5(1):e8799.

71. Libermann TA, Baltimore D. Activation of interleukin-6 gene expression through the NF-kappa B transcription factor. Mol Cell Biol. 1990;10(5):2327-2334.

72. Bonetti B, Stegagno C, Cannella B, Rizzuto N, Moretto G, Raine CS. Activation of NF- $\varkappa$ B and c-jun Transcription Factors in Multiple Sclerosis Lesions : Implications for Oligodendrocyte Pathology. The American Journal of Pathology. 1999;155(5):1433-1438.

73. Mc Guire C, Prinz M, Beyaert R, van Loo G. Nuclear factor kappa B (NF- $x$ B) in multiple sclerosis pathology. Trends Mol Med. 2013;19(10):604-613. doi: http://dx.doi.org/10.1016/j.molmed.2013.08.001.

74. Sen R, Baltimore D. Multiple nuclear factors interact with the immunoglobulin enhancer sequences. Cell. 1986;46(5):705-716. doi: 0092-8674(86)90346-6 [pii].

75. Gilmore TD. Introduction to NF-kappaB: players, pathways, perspectives. Oncogene. 2006;25(51):6680-6684. doi: 1209954 [pii]. 
76. Chen J, Chen ZJ. Regulation of NF-kappaB by ubiquitination. Curr Opin Immunol. 2013;25(1):4-12. doi: 10.1016/j.coi.2012.12.005 [doi].

77. Mattson MP, Camandola S. NF-kappaB in neuronal plasticity and neurodegenerative disorders. J Clin Invest. 2001;107(3):247-254. doi: 10.1172/JCI11916 [doi].

78. Pahan K, Schmid M. Activation of nuclear factor-kB in the spinal cord of experimental allergic encephalomyelitis. Neurosci Lett. 2000;287(1):17-20. doi: S03043940(00)01167-8 [pii].

79. Hilliard B, Samoilova EB, Liu TS, Rostami A, Chen Y. Experimental autoimmune encephalomyelitis in NF-kappa B-deficient mice:roles of NF-kappa B in the activation and differentiation of autoreactive T cells. J Immunol. 1999;163(5):2937-2943. doi: ji_v163n5p2937 [pii].

80. van Loo G, De Lorenzi R, Schmidt H, et al. Inhibition of transcription factor NFkappaB in the central nervous system ameliorates autoimmune encephalomyelitis in mice. Nat Immunol. 2006;7(9):954-961. doi: ni1372 [pii].

81. Hofman FM, Hinton DR, Johnson K, Merrill JE. Tumor necrosis factor identified in multiple sclerosis brain. J Exp Med. 1989;170(2):607-612.

82. Sharief MK, Hentges R. Association between tumor necrosis factor-alpha and disease progression in patients with multiple sclerosis. N Engl J Med. 1991;325(7):467-472. doi: 10.1056/NEJM199108153250704 [doi].

83. Ruddle NH, Bergman CM, McGrath KM, et al. An antibody to lymphotoxin and tumor necrosis factor prevents transfer of experimental allergic encephalomyelitis. $J$ Exp Med. 1990;172(4):1193-1200. 
84. The Lenercept Multiple Sclerosis Study Group and The University of British Columbia MS/MRI Analysis Group. TNF neutralization in MS: Results of a randomized, placebo-controlled multicenter study. Neurology. 1999;53(3):457-457. doi: 10.1212/WNL.53.3.457.

85. McCoy MK, Tansey MG. TNF signaling inhibition in the CNS: implications for normal brain function and neurodegenerative disease. Journal of Neuroinflammation. 2008;5(1):1-13. doi: 10.1186/1742-2094-5-45.

86. Arnett HA, Mason J, Marino M, Suzuki K, Matsushima GK, Ting JP. TNF alpha promotes proliferation of oligodendrocyte progenitors and remyelination. Nat Neurosci. 2001;4(11):1116-1122. doi: 10.1038/nn738 [doi].

87. Batoulis H, Recks MS, Holland FO, Thomalla F, Williams RO, Kuerten S. Blockade of tumour necrosis factor-alpha in experimental autoimmune encephalomyelitis reveals differential effects on the antigen-specific immune response and central nervous system histopathology. Clin Exp Immunol. 2014;175(1):41-48. doi: 10.1111/cei.12209 [doi].

88. Skurkovich S, Boiko A, Beliaeva I, et al. Randomized study of antibodies to IFNgamma and TNF-alpha in secondary progressive multiple sclerosis. Mult Scler. 2001;7(5):277284.

89. Maderna P, Godson C. Phagocytosis of apoptotic cells and the resolution of inflammation. Biochimica et Biophysica Acta (BBA) - Molecular Basis of Disease. 2003;1639(3):141-151. doi: http://dx.doi.org/10.1016/j.bbadis.2003.09.004.

90. Kinchen JM, Ravichandran KS. Phagosome maturation: going through the acid test. Nat Rev Mol Cell Biol. 2008;9(10):781-795. 
91. Auderset L, Landowski LM, Foa L, Young KM. Low Density Lipoprotein Receptor Related Proteins as Regulators of Neural Stem and Progenitor Cell Function. Stem Cells International. 2016;2016:2108495. doi: 10.1155/2016/2108495.

92. Sardiello M, Palmieri M, di Ronza A, et al. A Gene Network Regulating Lysosomal Biogenesis and Function. Science. 2009;325(5939):473-477. doi: 10.1126/science.1174447.

93. Neumann H, Kotter MR, Franklin RJM. Debris clearance by microglia: an essential link between degeneration and regeneration. Brain. 2008;132(2):288-295. doi: 10.1093/brain/awn109.

94. Kotter MR, Li W, Zhao C, Franklin RJM. Myelin Impairs CNS Remyelination by Inhibiting Oligodendrocyte Precursor Cell Differentiation. The Journal of Neuroscience. 2006;26(1):328-332.

95. Lampron A, Larochelle A, Laflamme N, et al. Inefficient clearance of myelin debris by microglia impairs remyelinating processes. The Journal of Experimental Medicine. 2015;212(4):481-495. doi: 10.1084/jem.20141656.

96. Boven LA, Van Meurs M, Van Zwam M, et al. Myelin-laden macrophages are antiinflammatory, consistent with foam cells in multiple sclerosis. Brain. 2006;129(2):517-526.

97. Fadok VA, Bratton DL, Konowal A, Freed PW, Westcott JY, Henson PM. Macrophages that have ingested apoptotic cells in vitro inhibit proinflammatory cytokine production through autocrine/paracrine mechanisms involving TGF $\beta$, PGE2, and PAF. $J$ Clin Invest. 1998;101(4):890-898. doi: 10.1172/JCI1112.

98. Voll RE, Herrmann M, Roth EA, Stach C, Kalden JR, Girkontaite I. Immunosuppressive effects of apoptotic cells. Nature. 1997;390(6658):350-351. 
99. Huynh MN, Fadok VA, Henson PM. Phosphatidylserine-dependent ingestion of apoptotic cells promotes TGF- $\beta$ secretion and the resolution of inflammation. $J$ Clin Invest. 2002;109(1):41-50. doi: 10.1172/JCI11638.

100. Chan A, Seguin R, Magnus T, et al. Phagocytosis of apoptotic inflammatory cells by microglia and its therapeutic implications: termination of CNS autoimmune inflammation and modulation by interferon-beta. Glia. 2003;43(3):231-242. doi: 10.1002/glia.10258.

101. Magnus T, Chan A, Grauer O, Toyka KV, Gold R. Microglial phagocytosis of apoptotic inflammatory $\mathrm{T}$ cells leads to down-regulation of microglial immune activation. $J$ Immunol. 2001;167(9):5004-5010.

102. Doyle SE, O'Connell R,M., Miranda GA, et al. Toll-like Receptors Induce a Phagocytic Gene Program through p38. J Exp Med. 2003;199(1):81-90. doi: 10.1084/jem.20031237.

103. Babcock AA, Wirenfeldt M, Holm T, et al. Toll-Like Receptor 2 Signaling in Response to Brain Injury: An Innate Bridge to Neuroinflammation. The Journal of Neuroscience. 2006;26(49):12826-12837. doi: 10.1523/JNEUROSCI.4937-05.2006.

104. Boivin A, Pineau I, Barrette B, et al. Toll-Like Receptor Signaling Is Critical for Wallerian Degeneration and Functional Recovery after Peripheral Nerve Injury. The Journal of Neuroscience. 2007;27(46):12565-12576. doi: 10.1523/JNEUROSCI.3027-07.2007.

105. Neniskyte U, Neher JJ, Brown GC. Neuronal Death Induced by Nanomolar Amyloid ß Is Mediated by Primary Phagocytosis of Neurons by Microglia. Journal of Biological Chemistry. 2011;286(46):39904-39913. doi: 10.1074/jbc.M111.267583. 
106. Sanjuan MA, Dillon CP, Tait SWG, et al. Toll-like receptor signalling in macrophages links the autophagy pathway to phagocytosis. Nature. 2007;450(7173):1253-1257.

107. Guerreiro R, Wojtas A, Bras J, et al. TREM2 Variants in Alzheimer's Disease. $N$ Engl J Med. 2013;368(2):117-127. doi: 10.1056/NEJMoa1211851.

108. Jonsson T, Stefansson H, Steinberg S, et al. Variant of TREM2 Associated with the Risk of Alzheimer's Disease. $N$ Engl J Med. 2013;368(2):107-116. doi: 10.1056/NEJMoa1211103.

109. Turnbull IR, Gilfillan S, Cella M, et al. Cutting Edge: TREM-2 Attenuates Macrophage Activation. The Journal of Immunology. 2006;177(6):3520-3524. doi: 10.4049/jimmunol.177.6.3520.

110. Hamerman JA, Jarjoura JR, Humphrey MB, Nakamura MC, Seaman WE, Lanier LL. Cutting Edge: Inhibition of TLR and FcR Responses in Macrophages by Triggering Receptor Expressed on Myeloid Cells (TREM)-2 and DAP12. The Journal of Immunology. 2006;177(4):2051-2055. doi: 10.4049/jimmunol.177.4.2051.

111. Takahashi K, Prinz M, Stagi M, Chechneva O, Neumann H. TREM2-transduced myeloid precursors mediate nervous tissue debris clearance and facilitate recovery in an animal model of multiple sclerosis. PLoS Med. 2007;4. doi: 10.1371/journal.pmed.0040124.

112. N'Diaye E, Branda CS, Branda SS, et al. TREM-2 (triggering receptor expressed on myeloid cells 2) is a phagocytic receptor for bacteria. The Journal of Cell Biology. 2009;184(2):215-223. doi: 10.1083/jcb.200808080. 
113. Hickman SE, El Khoury J. TREM2 and the neuroimmunology of Alzheimer's disease. Biochem $\quad$ Pharmacol. 2014;88(4):495-498. doi: http://dx.doi.org/10.1016/j.bcp.2013.11.021.

114. Jiang T, Tan L, Zhu X, et al. Upregulation of TREM2 Ameliorates Neuropathology and Rescues Spatial Cognitive Impairment in a Transgenic Mouse Model of Alzheimer/'s Disease. Neuropsychopharmacology. 2014;39(13):2949-2962.

115. Jay TR, Miller CM, Cheng PJ, et al. TREM2 deficiency eliminates TREM2+ inflammatory macrophages and ameliorates pathology in Alzheimer's disease mouse models. The Journal of Experimental Medicine. 2015;212(3):287-295. doi: 10.1084/jem.20142322.

116. Cantoni C, Bollman B, Licastro D, et al. TREM2 regulates microglial cell activation in response to demyelination in vivo. Acta Neuropathol. 2015;129(3):429-447. doi: 10.1007/s00401-015-1388-1 [doi].

117. Piccio L, Buonsanti C, Cella M, et al. Identification of soluble TREM-2 in the cerebrospinal fluid and its association with multiple sclerosis and CNS inflammation. Brain. 2008;131(11):3081-3091. doi: 10.1093/brain/awn217.

118. Piccio L, Buonsanti C, Mariani M, et al. Blockade of TREM-2 exacerbates experimental autoimmune encephalomyelitis. Eur J Immunol. 2007;37(5):1290-1301. doi: 10.1002/eji.200636837 [doi].

119. Herz J, Clouthier DE, Hammer RE. LDL receptor-related protein internalizes and degrades uPA-PAI-1 complexes and is essential for embryo implantation. Cell. 1992;71(3):411421. doi: 0092-8674(92)90511-A [pii]. 
120. Lillis AP, Van Duyn LB, Murphy-Ullrich JE, Strickland DK. LDL receptor-related protein 1: unique tissue-specific functions revealed by selective gene knockout studies. Physiol Rev. 2008;88(3):887-918. doi: 10.1152/physrev.00033.2007; 10.1152/physrev.00033.2007.

121. Kütt H, Herz J, Stanley KK. Structure of the low-density lipoprotein receptor-related protein (LRP) promoter. Biochimica et Biophysica Acta (BBA) - Gene Structure and Expression. 1989;1009(3):229-236. doi: http://dx.doi.org/10.1016/0167-4781(89)90107-3.

122. Emonard H, Théret L, Bennasroune AH, Dedieu S. Regulation of LRP-1 expression: Make the point. Pathologie Biologie. 2014;62(2):84-90. doi: http://dx.doi.org/10.1016/j.patbio.2014.02.002.

123. Willnow TE, Moehring JM, Inocencio NM, Moehring TJ, Herz J. The low-densitylipoprotein receptor-related protein (LRP) is processed by furin in vivo and in vitro. Biochem J. 1996;313 ( Pt 1)(Pt 1):71-76.

124. Reekmans SM, Pflanzner T, Gordts PLSM, et al. Inactivation of the proximal NPXY motif impairs early steps in LRP1 biosynthesis. Cellular and Molecular Life Sciences. 2010;67(1):135-145. doi: 10.1007/s00018-009-0171-7.

125. Gorovoy M, Gaultier A, Campana WM, Firestein GS, Gonias SL. Inflammatory mediators promote production of shed LRP1/CD91, which regulates cell signaling and cytokine expression by macrophages. J Leukoc Biol. 2010;88(4):769-778. doi: 10.1189/jlb.0410220 [doi].

126. Zemskov EA, Mikhailenko I, Strickland DK, Belkin AM. Cell-surface transglutaminase undergoes internalization and lysosomal degradation: an essential role for LRP1. J Cell Sci. 2007;120(Pt 18):3188-3199. doi: jcs.010397 [pii]. 
127. Yepes M, Sandkvist M, Moore EG, Bugge TH, Strickland DK, Lawrence DA. Tissue-type plasminogen activator induces opening of the blood-brain barrier via the LDL receptorâ€“"related protein. J Clin Invest. 2003;112(10):1533-1540. doi: 10.1172/JCI200319212.

128. Hochreiter-Hufford A, Ravichandran KS. Clearing the Dead: Apoptotic Cell Sensing, Recognition, Engulfment, and Digestion. Cold Spring Harbor Perspectives in Biology. 2013;5(1). doi: 10.1101/cshperspect.a008748.

129. Craig J, Mikhailenko I, Noyes N, Migliorini M, Strickland DK. The LDL ReceptorRelated Protein 1 (LRP1) Regulates the PDGF Signaling Pathway by Binding the Protein Phosphatase SHP-2 and Modulating SHP-2- Mediated PDGF Signaling Events. PLoS ONE. 2013;8(7):e70432.

130. Muratoglu SC, Mikhailenko I, Newton C, Migliorini M, Strickland DK. Low Density Lipoprotein Receptor-related Protein 1 (LRP1) Forms a Signaling Complex with Plateletderived Growth Factor Receptor- $\beta$ in Endosomes and Regulates Activation of the MAPK Pathway. Journal of Biological Chemistry. 2010;285(19):14308-14317. doi: 10.1074/jbc.M109.046672.

131. Gaultier A, Wu X, Le Moan N, et al. Low-density lipoprotein receptor-related protein 1 is an essential receptor for myelin phagocytosis. J Cell Sci. 2009;122(Pt 8):1155-1162. doi: $10.1242 /$ jcs.040717; $10.1242 /$ jcs.040717.

132. Stiles TL, Dickendesher TL, Gaultier A, et al. LDL receptor-related protein-1 is a sialic-acid-independent receptor for myelin-associated glycoprotein that functions in neurite outgrowth inhibition by MAG and CNS myelin. J Cell Sci. 2013;126(Pt 1):209-220. doi: $10.1242 /$ jcs.113191 [doi]. 
133. Fernandez-Castaneda A, Arandjelovic S, Stiles TL, et al. Identification of the Low Density Lipoprotein (LDL) Receptor-related Protein-1 Interactome in Central Nervous System Myelin Suggests a Role in the Clearance of Necrotic Cell Debris. Journal of Biological Chemistry. 2013;288(7):4538-4548. doi: 10.1074/jbc.M112.384693.

134. Gaultier A, Arandjelovic S, Niessen S, et al. Regulation of tumor necrosis factor receptor-1 and the IKK-NF- $x$ B pathway by LDL receptor-related protein explains the antiinflammatory activity of this receptor. Blood. 2008;111(11):5316-5325. doi: 10.1182/blood2007-12-127613.

135. Overton CD, Yancey PG, Major AS, Linton MF, Fazio S. Deletion of Macrophage LDL Receptor-Related Protein Increases Atherogenesis in the Mouse. Circulation Research. 2007;100(5):670-677. doi: 10.1161/01.RES.0000260204.40510.aa.

136. Gaultier A, Arandjelovic S, Li X, et al. A shed form of LDL receptor-related protein-1 regulates peripheral nerve injury and neuropathic pain in rodents. J Clin Invest. 2008;118(1):161-172. doi: 10.1172/JCI32371 [doi].

137. Mantuano E, Brifault C, Lam MS, Azmoon P, Gilder AS, Gonias SL. LDL receptorrelated protein-1 regulates $\mathrm{NF} x \mathrm{~B}$ and microRNA-155 in macrophages to control the inflammatory response. Proceedings of the National Academy of Sciences. 2016;113(5):13691374. doi: 10.1073/pnas.1515480113.

138. Staudt ND, Jo M, Hu J, et al. Myeloid cell receptor LRP1/CD91 regulates monocyte recruitment and angiogenesis in tumors. Cancer Res. 2013;73(13):3902-3912. doi: 10.1158/00085472.CAN-12-4233 [doi]. 
139. May P. The low-density lipoprotein receptor-related protein 1 in inflammation. Curr Opin Lipidol. 2013;24(2):134-137. doi: 10.1097/MOL.0b013e32835e809c; 10.1097/MOL.0b013e32835e809c.

140. Zhu L, Giunzioni I, Tavori H, et al. Loss of Macrophage Low-Density Lipoprotein Receptor-Related Protein 1 Confers Resistance to the Antiatherogenic Effects of Tumor Necrosis Factor-alpha Inhibition. Arterioscler Thromb Vasc Biol. 2016. doi: ATVBAHA.116.307736 [pii].

141. Zurhove K, Nakajima C, Herz J, Bock HH, May P. Gamma-secretase limits the inflammatory response through the processing of LRP1. Sci Signal. 2008;1(47):ra15. doi: 10.1126/scisignal.1164263; 10.1126/scisignal.1164263.

142. Huang SC, Everts B, Ivanova Y, et al. Cell-intrinsic lysosomal lipolysis is essential for alternative activation of macrophages. Nat Immunol. 2014;15(9):846-855.

143. May,Petra, Bock,HansH., Nofer,Jerzy-Roch. Low density receptor-related protein 1 (LRP1) promotes anti-inflammatory phenotype in murine macrophages.

144. O'Connell RM, Taganov KD, Boldin MP, Cheng G, Baltimore D. MicroRNA-155 is induced during the macrophage inflammatory response. Proceedings of the National Academy of Sciences. 2007;104(5):1604-1609. doi: 10.1073/pnas.0610731104.

145. Rosenbloom KR, Armstrong J, Barber GP, et al. The UCSC Genome Browser database: 2015 update. Nucleic Acids Res. 2015;43(Database issue):D670-81. doi: 10.1093/nar/gku1177 [doi].

146. Zhang Y, Chen K, Sloan SA, et al. An RNA-sequencing transcriptome and splicing database of glia, neurons, and vascular cells of the cerebral cortex. $J$ Neurosci. 2014;34(36):11929-11947. doi: 10.1523/JNEUROSCI.1860-14.2014 [doi]. 
147. Lewis ND, Hill JD, Juchem KW, Stefanopoulos DE, Modis LK. RNA sequencing of microglia and monocyte-derived macrophages from mice with experimental autoimmune encephalomyelitis illustrates a changing phenotype with disease course. J Neuroimmunol. 2014;277(1-2):26-38. doi: 10.1016/j.jneuroim.2014.09.014 [doi].

148. Yamada K, Hashimoto T, Yabuki C, et al. The Low Density Lipoprotein Receptorrelated Protein 1 Mediates Uptake of Amyloid ß Peptides in an in Vitro Model of the Blood-Brain Barrier Cells. Journal of Biological Chemistry. 2008;283(50):34554-34562. doi: 10.1074/jbc.M801487200.

149. Ito S, Ohtsuki S, Kamiie J, Nezu Y, Terasaki T. Cerebral clearance of human amyloid-? peptide (1?40) across the blood?brain barrier is reduced by self-aggregation and formation of low-density lipoprotein receptor-related protein-1 ligand complexes. J Neurochem. 2007;103(6):2482-2490. doi: 10.1111/j.1471-4159.2007.04938.x.

150. Storck SE, Meister S, Nahrath J, et al. Endothelial LRP1 transports amyloid- $\beta 1-42$ across the blood-brain barrier. J Clin Invest. 2016;126(1):123-136. doi: 10.1172/JCI81108.

151. BELL RD, WINKLER EA, SINGH I, et al. Apolipoprotein E controls cerebrovascular integrity via cyclophilin A. Nature. 2012;485(7399):512-516. doi: 10.1038/nature 11087 .

152. Barnett MH, Prineas JW. Relapsing and remitting multiple sclerosis: pathology of the newly forming lesion. Ann Neurol. 2004;55(4):458-468. doi: 10.1002/ana.20016 [doi].

153. Hendrickx DA, Koning N, Schuurman KG, et al. Selective upregulation of scavenger receptors in and around demyelinating areas in multiple sclerosis. $J$ Neuropathol Exp Neurol. 2013;72(2):106-118. doi: 10.1097/NEN.0b013e31827fd9e8 [doi]. 
154. Su AI, Wiltshire T, Batalov S, et al. A gene atlas of the mouse and human proteinencoding transcriptomes. Proc Natl Acad Sci U S A. 2004;101(16):6062-6067. doi: 10.1073/pnas.0400782101 [doi].

155. Popescu BF, Pirko I, Lucchinetti CF. Pathology of multiple sclerosis: where do we stand?. Continuum (Minneap Minn). 2013;19(4 Multiple Sclerosis):901-921. doi: 10.1212/01.CON.0000433291.23091.65 [doi].

156. Selmaj KW, Raine CS. Experimental autoimmune encephalomyelitis: immunotherapy with anti-tumor necrosis factor antibodies and soluble tumor necrosis factor receptors. Neurology. 1995;45(6 Suppl 6):S44-9.

157. King IL, Dickendesher TL, Segal BM. Circulating Ly-6C+ myeloid precursors migrate to the CNS and play a pathogenic role during autoimmune demyelinating disease. Blood. 2009;113(14):3190-3197. doi: 10.1182/blood-2008-07-168575; 10.1182/blood-2008-07-168575.

158. Vainchtein ID, Vinet $\mathrm{J}$, Brouwer $\mathrm{N}$, et al. In acute experimental autoimmune encephalomyelitis, infiltrating macrophages are immune activated, whereas microglia remain immune suppressed. Glia. 2014;62(10):1724-1735. doi: 10.1002/glia.22711 [doi].

159. Goldmann T, Wieghofer P, Muller PF, et al. A new type of microglia gene targeting shows TAK1 to be pivotal in CNS autoimmune inflammation. Nat Neurosci. 2013;16(11):16181626. doi: $10.1038 / \mathrm{nn} .3531$ [doi].

160. Gardai SJ, McPhillips KA, Frasch SC, et al. Cell-surface calreticulin initiates clearance of viable or apoptotic cells through trans-activation of LRP on the phagocyte. Cell. 2005;123(2):321-334. doi: 10.1016/j.cell.2005.08.032. 
161. Hilliard BA, Mason $\mathrm{N}, \mathrm{Xu} \mathrm{L}$, et al. Critical roles of c-Rel in autoimmune inflammation and helper $\mathrm{T}$ cell differentiation. J Clin Invest. 2002;110(6):843-850. doi: 10.1172/JCI15254 [doi].

162. Wolf BB, Lopes MB, VandenBerg SR, Gonias SL. Characterization and immunohistochemical localization of alpha 2-macroglobulin receptor (low-density lipoprotein receptor-related protein) in human brain. The American Journal of Pathology. 1992;141(1):37-42.

163. Rohlmann A, Gotthardt M, Willnow TE, Hammer RE, Herz J. Sustained somatic gene inactivation by viral transfer of Cre recombinase. Nat Biotech. 1996;14(11):1562-1565.

164. Subramanian M, Hayes CD, Thome JJ, et al. An AXL/LRP-1/RANBP9 complex mediates DC efferocytosis and antigen cross-presentation in vivo. J Clin Invest. 2014. doi: 10.1172/JCI72051; 10.1172/JCI72051.

165. Bennett J, Basivireddy J, Kollar A, et al. Blood-brain barrier disruption and enhanced vascular permeability in the multiple sclerosis model EAE. J Neuroimmunol. 2010;229(1-2):180-191. doi: http://dx.doi.org/10.1016/j.jneuroim.2010.08.011.

166. Fonseca ACCD, Matias DIL, Garcia C, et al. The impact of microglial activation on blood-brain barrier in brain diseases. Frontiers in Cellular Neuroscience. 2014;8.

167. Ramirez SH, Haskó J, Skuba A, et al. Activation of Cannabinoid Receptor 2 Attenuates Leukocyte-Endothelial Cell Interactions and Blood-Brain Barrier Dysfunction under Inflammatory Conditions. The Journal of Neuroscience. 2012;32(12):4004-4016. doi: 10.1523/JNEUROSCI.4628-11.2012. 
168. Chen Z, Jalabi W, Shpargel KB, et al. Lipopolysaccharide-induced microglial activation and neuroprotection against experimental brain injury is independent of hematogenous TLR4. J Neurosci. 2012;32(34):11706-11715 . doi: 10.1523/JNEUROSCI.0730-12.2012 [doi].

169. Lu YC, Yeh WC, Ohashi PS. LPS/TLR4 signal transduction pathway. Cytokine. 2008;42(2):145-151. doi: 10.1016/j.cyto.2008.01.006 [doi].

170. Yu L, Wang L, Chen S. Endogenous toll-like receptor ligands and their biological significance. J Cell Mol Med. 2010;14(11):2592-2603. doi: 10.1111/j.1582-4934.2010.01127.x [doi].

171. Lopes MB, Bogaev CA, Gonias SL, VandenBerg SR. Expression of alpha 2macroglobulin receptor/low density lipoprotein receptor-related protein is increased in reactive and neoplastic glial cells. FEBS Lett. 1994;338(3):301-305. doi: 0014-5793(94)80288-2 [pii].

172. Correale J, Farez MF. The Role of Astrocytes in Multiple Sclerosis Progression. Frontiers in Neurology. 2015;6:180. doi: 10.3389/fneur.2015.00180.

173. D'Mello C, Le T, Swain MG. Cerebral microglia recruit monocytes into the brain in response to tumor necrosis factoralpha signaling during peripheral organ inflammation. $J$ Neurosci. 2009;29(7):2089-2102. doi: 10.1523/JNEUROSCI.3567-08.2009 [doi].

174. Kozlowski C, Weimer RM. An automated method to quantify microglia morphology and application to monitor activation state longitudinally in vivo. PLoS One. 2012;7(2):e31814. doi: 10.1371/journal.pone.0031814 [doi].

175. Su AI, Cooke MP, Ching KA, et al. Large-scale analysis of the human and mouse transcriptomes. Proc Natl Acad Sci U S A. 2002;99(7):4465-4470. doi: 10.1073/pnas.012025199 [doi]. 
176. Chuang T, Guo Y, Seki SM, et al. LRP1 expression in microglia is protective during CNS autoimmunity. Acta Neuropathologica Communications. 2016;4(1):1-12. doi: 10.1186/s40478-016-0343-2.

177. Garcia JA, Pino PA, Mizutani M, et al. Regulation of Adaptive Immunity by the Fractalkine Receptor during Autoimmune Inflammation. The Journal of Immunology. 2013;191(3):1063-1072. doi: 10.4049/jimmunol.1300040.

178. Haskell CA, Hancock WW, Salant DJ, et al. Targeted deletion of CX3CR1 reveals a role for fractalkine in cardiac allograft rejection. J Clin Invest. 2001;108(5):679-688. doi: 10.1172/JCI12976.

179. Huang D, Shi F, Jung S, et al. The neuronal chemokine CX3CL1/fractalkine selectively recruits NK cells that modify experimental autoimmune encephalomyelitis within the central nervous system. The FASEB Journal. 2006;20(7):896-905. doi: 10.1096/fj.05-5465com.

180. Lou N, Takano T, Pei Y, Xavier AL, Goldman SA, Nedergaard M. Purinergic receptor P2RY12-dependent microglial closure of the injured blood-brain barrier. Proceedings of the National Academy of Sciences. 2016;113(4):1074-1079. doi: 10.1073/pnas.1520398113.

181. Agrawal SM, Williamson J, Sharma R, et al. Extracellular matrix metalloproteinase inducer shows active perivascular cuffs in multiple sclerosis. Brain. 2013. doi: 10.1093/brain/awt093.

182. Morrison HW, Filosa JA. A quantitative spatiotemporal analysis of microglia morphology during ischemic stroke and reperfusion. Journal of Neuroinflammation. 2013;10(1):1-20. doi: 10.1186/1742-2094-10-4. 
183. Takaesu G, Kishida S, Hiyama A, et al. TAB2, a novel adaptor protein, mediates activation of TAK1 MAPKKK by linking TAK1 to TRAF6 in the IL-1 signal transduction pathway. Mol Cell. 2000;5(4):649-658. doi: S1097-2765(00)80244-0 [pii].

184. Kishida S, Sanjo H, Akira S, Matsumoto K, Ninomiya-Tsuji J. TAK1-binding protein 2 facilitates ubiquitination of TRAF6 and assembly of TRAF6 with IKK in the IL-1 signaling pathway. Genes Cells. 2005;10(5):447-454. doi: GTC852 [pii].

185. Landström M. The TAK1-TRAF6 signalling pathway. Int J Biochem Cell Biol. 2010;42(5):585-589. doi: http://dx.doi.org/10.1016/j.biocel.2009.12.023.

186. Muroi M, Tanamoto K. TRAF6 distinctively mediates MyD88- and IRAK-1induced activation of NF-kappaB. J Leukoc Biol. 2008;83(3):702-707. doi: jlb.0907629 [pii].

187. Jiao S, Zhang Z, Li C, et al. The kinase MST4 limits inflammatory responses through direct phosphorylation of the adaptor TRAF6. Nat Immunol. 2015;16(3):246-257.

188. Inoue J, Gohda J, Akiyama T. Characteristics and Biological Functions of TRAF6. In: Wu H, ed. TNF Receptor Associated Factors (TRAFs). New York, NY: Springer New York; 2007:72-79.

189. Cao Z, Henzel WJ, Gao X. IRAK: a kinase associated with the interleukin-1 receptor. Science. 1996;271(5252):1128-1131.

190. Tang L, Zhou X, Wang Q, et al. Expression of TRAF6 and pro-inflammatory cytokines through activation of TLR2, TLR4, NOD1, and NOD2 in human periodontal ligament fibroblasts. Arch $\quad$ Oral $\quad$ Biol. 2011;56(10):1064-1072. doi: http://dx.doi.org/10.1016/j.archoralbio.2011.02.020. 
191. Zhu Z, Wang L, Hao R, Zhao B, Sun L, Ye RD. Cutting Edge: A Cullin-5-TRAF6 Interaction Promotes TRAF6 Polyubiquitination and Lipopolysaccharide Signaling. The Journal of Immunology. 2016. doi: 10.4049/jimmunol.1600447.

192. Zhong J, Kyriakis JM. Germinal center kinase is required for optimal Jun N-terminal kinase activation by Toll-like receptor agonists and is regulated by the ubiquitin proteasome system and agonist-induced, TRAF6-dependent stabilization. Mol Cell Biol. 2004;24(20):91659175. doi: 10.1128/MCB.24.20.9165-9175.2004 [doi].

193. Söderberg O, Leuchowius K, Gullberg M, et al. Characterizing proteins and their interactions in cells and tissues using the in situ proximity ligation assay. Methods. 2008;45(3):227-232. doi: http://dx.doi.org/10.1016/j.ymeth.2008.06.014.

194. Fredriksson S, Gullberg M, Jarvius J, et al. Protein detection using proximitydependent DNA ligation assays.

195. Catrysse L, Vereecke L, Beyaert R, van Loo G. A20 in inflammation and autoimmunity. Trends Immunol. ;35(1):22-31 . doi: 10.1016/j.it.2013.10.005.

196. Lee EG, Boone DL, Chai S, et al. Failure to Regulate TNF-Induced NF- $\hat{I}^{\circ} B$ and Cell Death Responses in A20-Deficient Mice. Science. 2000;289(5488):2350-2354.

197. Verstrepen L, Verhelst K, van Loo G, Carpentier I, Ley SC, Beyaert R. Expression, biological activities and mechanisms of action of A20 (TNFAIP3). Biochem Pharmacol. 2010;80(12):2009-2020. doi: http://dx.doi.org/10.1016/j.bcp.2010.06.044.

198. Gveric D, Kaltschmidt C, Cuzner ML, Newcombe J. Transcription factor NFkappaB and inhibitor I kappaBalpha are localized in macrophages in active multiple sclerosis lesions. J Neuropathol Exp Neurol. 1998;57(2):168-178. 
199. Kaltschmidt B, Kaltschmidt C. NF- B in the Nervous System. Cold Spring Harbor Perspectives in Biology. 2009;1(3). doi: 10.1101/cshperspect.a001271.

200. Dasgupta S, Jana M, Zhou Y, Fung YK, Ghosh S, Pahan K. Antineuroinflammatory effect of NF-kappaB essential modifier-binding domain peptides in the adoptive transfer model of experimental allergic encephalomyelitis. $J$ Immunol. 2004;15;173. doi: 10.4049/jimmunol.173.2.1344.

201. Tischner D, Reichardt HM. Glucocorticoids in the control of neuroinflammation. Mol Cell Endocrinol. 2007;275(1-2):62-70. doi: http://dx.doi.org/10.1016/j.mce.2007.03.007.

202. Clarner T, Diederichs F, Berger K, et al. Myelin debris regulates inflammatory responses in an experimental demyelination animal model and multiple sclerosis lesions. Glia. 2012;60(10):1468-1480. doi: 10.1002/glia.22367 [doi].

203. Natrajan MS, Komori M, Kosa P, et al. Pioglitazone regulates myelin phagocytosis and multiple sclerosis monocytes. Ann Clin Transl Neurol. 2015;2(12):1071-1084. doi: 10.1002/acn3.260.

204. Xiao Y, Jin J, Chang $M$, et al. Peli1 promotes microglia-mediated CNS inflammation by regulating Traf3 degradation. Nat Med. 2013;19(5):595-602.

205. Sierra A, Abiega O, Shahraz A, Neumann H. Janus-faced microglia: beneficial and detrimental consequences of microglial phagocytosis. Frontiers in Cellular Neuroscience. $2013 ; 7$.

206. Rinner WA, Bauer J, Schmidts M, Lassmann H, Hickey WF. Resident microglia and hematogenous macrophages as phagocytes in adoptively transferred experimental autoimmune 
encephalomyelitis: an investigation using rat radiation bone marrow chimeras. Glia. 1995;14(4):257-266. doi: 10.1002/glia.440140403 [doi].

207. Prinz M, Priller J, Sisodia SS, Ransohoff RM. Heterogeneity of CNS myeloid cells and their roles in neurodegeneration. Nat Neurosci. 2011;14(10):1227-1235.

208. Biber K, Owens T, Boddeke E. What is microglia neurotoxicity (Not)?. Glia. 2014;62(6):841-854. doi: 10.1002/glia.22654.

209. Beutner C, Linnartz-Gerlach B, Schmidt SV, et al. Unique transcriptome signature of mouse microglia. Glia. 2013;61(9):1429-1442. doi: 10.1002/glia.22524.

210. Baer AS, Syed YA, Kang SU, et al. Myelin-mediated inhibition of oligodendrocyte precursor differentiation can be overcome by pharmacological modulation of Fyn-RhoA and protein kinase C signalling. Brain. 2008;132(2):465-481. doi: 10.1093/brain/awn334.

211. Rivest S. CX3CR1 in multiple sclerosis. Oncotarget. 2015;6(24):19946-19947.

212. Appelqvist H, Wäster P, Kågedal K, Öllinger K. The lysosome: from waste bag to potential therapeutic target. Journal of Molecular Cell Biology. 2013;5(4):214-226. doi: 10.1093/jmcb/mjt022.

213. Nayak JV, Hokey DA, Larregina A, et al. Phagocytosis Induces Lysosome Remodeling and Regulated Presentation of Particulate Antigens by Activated Dendritic Cells. The Journal of Immunology. 2006;177(12):8493-8503. doi: 10.4049/jimmunol.177.12.8493.

214. Shen K, Sidik H, Talbot W. The Rag-Ragulator Complex Regulates Lysosome Function and Phagocytic Flux in Microglia. Cell Reports. ;14(3):547-559. doi: 10.1016/j.celrep.2015.12.055. 
215. Li H, Newcombe J, Groome NP, Cuzner ML. Characterization and distribution of phagocytic macrophages in multiple sclerosis plaques. Neuropathol Appl Neurobiol. 1993;19(3):214-223.

216. Huizinga R, van der Star BJ, Kipp M, et al. Phagocytosis of neuronal debris by microglia is associated with neuronal damage in multiple sclerosis. Glia. 2012;60(3):422-431. doi: 10.1002/glia.22276 [doi].

217. Bishop DL, Misgeld T, Walsh MK, Gan WB, Lichtman JW. Axon branch removal at developing synapses by axosome shedding. Neuron. 2004;44(4):651-661. doi: S0896627304007111 [pii].

218. David S, Lacroix S. Molecular approaches to spinal cord repair. Annu Rev Neurosci. 2003;26:411-440. doi: 10.1146/annurev.neuro.26.043002.094946 [doi].

219. Becerra JL, Puckett WR, Hiester ED, et al. MR-pathologic comparisons of wallerian degeneration in spinal cord injury. AJNR Am J Neuroradiol. 1995;16(1):125-133.

220. Buss A, Brook GA, Kakulas B, et al. Gradual loss of myelin and formation of an astrocytic scar during Wallerian degeneration in the human spinal cord. Brain. 2004;127(Pt 1):3444. doi: 10.1093/brain/awh001 [doi].

221. Gaudet AD, Popovich PG, Ramer MS. Wallerian degeneration: gaining perspective on inflammatory events after peripheral nerve injury. Journal of Neuroinflammation. 2011;8(1):113. doi: 10.1186/1742-2094-8-110.

222. Napolitano G, Ballabio A. TFEB at a glance. J Cell Sci. 2016. 
223. Chauhan S, Ahmed Z, Bradfute SB, et al. Pharmaceutical screen identifies novel target processes for activation of autophagy with a broad translational potential. Nat Commun. $2015 ; 6$.

224. Polito VA, Li H, Martiniâ€ Stoica H, et al. Selective clearance of aberrant tau proteins and rescue of neurotoxicity by transcription factor EB. EMBO Molecular Medicine. 2014;6(9):1142-1160.

225. Xiao Q, Yan P, Ma X, et al. Neuronal-Targeted TFEB Accelerates Lysosomal Degradation of APP, Reducing A $\beta$ Generation and Amyloid Plaque Pathogenesis. The Journal of Neuroscience. 2015;35(35):12137-12151. doi: 10.1523/JNEUROSCI.0705-15.2015.

226. Hendrickx DAE, Schuurman KG, van Draanen M, Hamann J, Huitinga I. Enhanced uptake of multiple sclerosis-derived myelin by THP-1 macrophages and primary human microglia. Journal of Neuroinflammation. 2014;11(1):1-11. doi: 10.1186/1742-2094-11-64.

227. Pul R, Morbiducci F, Å kuljec J, et al. Glatiramer Acetate Increases Phagocytic Activity of Human Monocytes In Vitro and in Multiple Sclerosis Patients. PLoS ONE. 2012;7(12):e51867. 